\title{
Keeping it real: understanding and changing health behavior in daily life
}

\author{
Citation for published version (APA):
}

Spook, J. E. (2016). Keeping it real: understanding and changing health behavior in daily life. [Doctoral Thesis, Maastricht University]. Maastricht University. https://doi.org/10.26481/dis.20160318js

Document status and date:

Published: 01/01/2016

DOI:

10.26481/dis.20160318js

Document Version:

Publisher's PDF, also known as Version of record

\section{Please check the document version of this publication:}

- A submitted manuscript is the version of the article upon submission and before peer-review. There can be important differences between the submitted version and the official published version of record.

People interested in the research are advised to contact the author for the final version of the publication, or visit the DOI to the publisher's website.

- The final author version and the galley proof are versions of the publication after peer review.

- The final published version features the final layout of the paper including the volume, issue and page numbers.

Link to publication

\footnotetext{
General rights rights.

- You may freely distribute the URL identifying the publication in the public portal. please follow below link for the End User Agreement:

www.umlib.nl/taverne-license

Take down policy

If you believe that this document breaches copyright please contact us at:

repository@maastrichtuniversity.nl

providing details and we will investigate your claim.
}

Copyright and moral rights for the publications made accessible in the public portal are retained by the authors and/or other copyright owners and it is a condition of accessing publications that users recognise and abide by the legal requirements associated with these

- Users may download and print one copy of any publication from the public portal for the purpose of private study or research.

- You may not further distribute the material or use it for any profit-making activity or commercial gain

If the publication is distributed under the terms of Article $25 \mathrm{fa}$ of the Dutch Copyright Act, indicated by the "Taverne" license above, 


\section{KEEPING IT REAL: Understanding and Changing Health Behavior in Daily Life}




\section{KEEPING IT REAL: \\ Understanding and Changing Health Behavior in Daily Life}




\section{COLOPHON}

The research presented in this dissertation was conducted at TNO, Life Style expertise centre, and carried out in collaboration with Maastricht University, Work and Social Psychology department.

Cover design: Jillisa en Jorinde Spook

Layout: Vincent van Zandvoord (Vormvast Creative Centre In Design) and Jorinde Spook Production: PrintSupport4U, Meppel, The Netherlands

ISBN: 9789082196825

(c) 2016 Jorinde Eline Spook, Leiden

All rights reserved. No parts of this thesis may be reproduced or transmitted in any form or by any means, electronic or mechanical, including photocopy, recording or any other information storage or retrieval system, without permission in writing from the author, or, when appropriate, from the publishers holding the copyright of the published articles. 


\section{KEEPING IT REAL:}

Understanding and Changing Health Behavior in Daily Life

PROEFSCHRIFT

ter verkrijgen van de graad van doctor aan de Universiteit Maastricht, op gezag van de Rector Magnificus, Prof. Dr. L.L.G. Soete, volgens het besluit van het College van Decanen,

in het openbaar te verdedigen op vrijdag 18 maart 2016 om 10.00 uur

door

Jorinde Eline Spook 


\section{Promotor}

Prof. dr. G. Kok

\section{Copromotoren}

Dr. P. van Empelen (TNO)

Dr. T. Paulussen (TNO)

\section{Beoordelingscommissie}

Prof. dr. R.A.C. Ruiter (voorzitter)

Dr. R. Crutzen

Prof. dr. S. Kremers

Prof. dr. L. Lechner (Open Universiteit)

Dr. E.W.M.L. de Vet (Wageningen University \& Research centre) 


\section{TABLE OF CONTENTS}

Chapter 1: General Introduction

Chapter 2: Feasibility, Usability, and Ecological Validity of a mobile-based Ecological Momentary Assessment (mEMA) Tool: Monitoring Dietary Intake and Physical Activity Electronically. Journal of Medical Internet Research, 2013, 15(9), e214.

Chapter 3: The role of Motivational and Contextual Factors on Intentional and Craving48 Induced Snack Consumption: A mobile-based Ecological Momentary Assessment Study. Submitted for Publication.

Chapter 4: Factors Determining Physical Activity of Emerging Young Adults: A mobile70 based Ecological Momentary Assessment (mEMA) Study. Submitted for Publication.

Chapter 5: Design rationale behind the serious self-regulation game intervention 92 "Balance It": Overweight prevention among secondary vocational education students in the Netherlands. Games for Health Journal, 2015, 4(6), 1-14.

Chapter 6: Evaluation of "Balance It": A pilot study on the effects of a serious selfregulation game intervention for overweight-related behaviors. Under Review.

Chapter 7: General Discussion

Valorisation Addendum

Summary

Samenvatting 194

Dankwoord 202

Curriculum vitae 206 



\section{CHAPTER 1}

General Introduction 


\section{General Introduction}

Over the past few decades, the prevalence of overweight (Body Mass Index (BMI) 25-30) and obesity (BMI $\geq 30$ ) has substantially increased in developed countries (World Health Organization [WHO], 2013a). On a global level, approximately $39 \%$ of adults are overweight, and $13 \%$ are obese. These percentages reflect the 1.9 billion adults who are overweight, and the 600 million of these with obesity (World Health Organization [WHO], 2013a). In the Netherlands, approximately 52\% of the Dutch adult population is overweight, and another $18.8 \%$ individuals are obese (World Health Organization [WHO], 2013b). Unfortunately, excess weight not only affects physical appearance, it can lead to serious physical and mental health problems, and has been recognized as a disease (AMA, 2013; Rubenstein, 2005).

Health problems that are associated with excess weight include type 2 diabetes mellitus, cardiovascular disease, osteoarthritis, some cancers, and mental illnesses such as depression and anxiety disorders (Rubenstein, 2005; European Heart Initiative, 2001). Consequently, increasing numbers of health issues associated with overweight and obesity drive up healthcare costs (Polder \& Takkern, 2002; Wang, McPherson, Marsh, Gortmaker, \& Brown, 2011). The increasing prevalence of overweight and obesity is most evident in low socio-economic status (SES) families and minority groups (Schönbeck \& van Buuren, 2010; Wang, Monteiro, \& Popkin, 2002; Wang et al., 2007). As being overweight during adulthood is strongly predicted by being overweight during childhood (Maffeis \& Tato, 2001; Must, 2003; Seidell, 2000), it is important to focus intervention efforts on youth from low SES families and from minority groups. In the Netherlands, about $16 \%$ of Dutch youth with a Dutch background are overweight or obese, as compared to $34-40 \%$ of youth with a Moroccan or Turkish background (Schönbeck \& van Buuren, 2010). As many low SES youth participate in secondary vocational education (Dutch “Middelbaar Beroeps Opleiding”, or MBO; Kerncijfers 2007-2011, 2012), we mainly focused on this group in the studies presented in this dissertation.

\section{The (Secondary Vocational) Education System in the Netherlands}

All children in the Netherlands start primary school at the age of four years old and stay in primary school until the age of approximately eleven or twelve years old. After a national examination at the end of primary school, children move on to secondary education. Based on their examination scores and advice from their primary school teacher during the final year, they will be allocated either to preparatory secondary vocational education (a four year program), senior general secondary educa- 
tion (a five year program), or pre-university education (a six year program). These secondary education programs prepare them for vocational education, higher professional education, and university, respectively. As the present study focuses on vocational education students, the vocational education system will be described in more detail.

Secondary vocational education (Dutch "MBO") prepares students for a wide range of occupations and includes four different levels of training: assistant training (level 1), basic vocational training (level 2), professional training (level 3), and middle-management training (level 4). Each level contains a vocational training pathway consisting of $20-60 \%$ practical training. Holders of a level 4 certificate may continue with professional education (Dutch "HBO") if desired.

In 2013, the secondary vocational education system consisted of 69 schools in the Netherlands, teaching 514.500 students over 1092 different training courses (MBO Raad, 2014). At this time, 21.000 students (level 1), 113.500 students (level 2), 140.000 students (level 3), and 240.000 students (level 4) were enrolled in secondary vocational education. Most of the students (71\%) were full-time vocational training students and were mainly studying economics (33\%), health care (32\%), and engineering (27\%). Overall, the majority of Dutch students are enrolled in secondary vocational education (44\%), followed by higher professional training (36\%) and university (20\%) (DUO, 2013). Given the extensive number of secondary vocational education students who have a relatively higher risk of becoming overweight or obese, studying overweight-related behaviors and the factors that influence these behaviors is key to weight-gain prevention. The Intervention Mapping (IM) protocol (Bartholomew, Parcel, Kok, Gottlieb, \& Fernández, 2011) provides a useful and relevant framework for the entire process of developing behavior change strategies.

\section{Systematic Intervention Development: An Intervention Mapping Approach}

The Intervention Mapping (IM) protocol describes the six steps involved in systematically planning a theory- and evidence-based intervention (see Figure 1). The protocol starts with a needs assessment (step 1), in which the health problem, the behavioral and environmental causes of this problem, and any determinants of these causes are assessed. In step 2 of IM, the change objectives for the intervention are specified. Change objectives specify what needs to be changed with regard to a specific determinant in order to be able to perform the desired behavior (i.e., the performance objectives). In step 3, theory- and evidence-based behavior change methods or techniques are translated into 
practical applications. Notably, these methods refer to the theoretical techniques by which behavior (or determinants of behavior) is (or are) expected to change. Importantly, the theory provides the requirements that need to be met for the effective use of a technique. Step 4 concerns the logical integration of the previously selected applications into a coherent intervention program design. In step 5, implementation objectives are specified for program adoption, implementation, and sustainability. Subsequently, determinants for adoption and implementation are specified, change objectives are formulated, and methods are selected to improve the adoption and implementation of the intervention. Finally, in step 6, an evaluation plan is conducted in order to validate the intervention, describing the program outcomes, evaluation questions, and the related measures (Bartholomew et al., 2011). 


\begin{tabular}{|c|c|c|}
\hline \multirow{6}{*}{ Evaluation } & $\begin{array}{l}\text { Step } 1 \\
\text { Needs Assessment }\end{array}$ & $\begin{array}{l}\text { - Plan needs assessment or problem analysis } \\
\text { - Assess health, quality of life, behaviour, and environment } \\
\text { - Assess capacity } \\
\text { - Establish programme outcomes }\end{array}$ \\
\hline & $\begin{array}{l}\text { Step } 2 \\
\text { Matrices }\end{array}$ & $\begin{array}{l}\text { - State expected changes in behaviour and environment } \\
\text { - Specify performance objectives } \\
\text { - Specify determinants of the target behaviour of the at risk } \\
\text { group } \\
\text { - Create matrices of change objectives }\end{array}$ \\
\hline & $\begin{array}{l}\text { Step } 3 \\
\text { Theory-Based Methods } \\
\text { and Practical Strategies }\end{array}$ & $\begin{array}{l}\text { - Review programme ideas with interested participants } \\
\text { - Identify theoretical methods } \\
\text { - Choose programme methods } \\
\text { - Select or design strategies } \\
\text { - Ensure that strategies match change objectives }\end{array}$ \\
\hline & $\begin{array}{l}\text { Step } 4 \\
\text { Programme }\end{array}$ & $\begin{array}{l}\text { - Consult with intended participants and implementers } \\
\text { - Create programme scope, sequence, theme, and materials list } \\
\text { - Develop design documents and protocols } \\
\text { - Review available materials } \\
\text { - Develop programme materials } \\
\text { - Pretest programme materials with target groups and } \\
\text { implementers and oversee materials production }\end{array}$ \\
\hline & $\begin{array}{l}\text { Step } 5 \\
\text { Adoption and } \\
\text { Implementation } \\
\text { Plan }\end{array}$ & $\begin{array}{l}\text { - Identify adopters and users } \\
\text { - specify adoption, implementation, and sustainability } \\
\text { performance objectives } \\
\text { - Specify determinants and create matrix } \\
\text { - Select methods and strategies } \\
\text { - Design interventions to affect programme use }\end{array}$ \\
\hline & $\begin{array}{l}\text { Step } 6 \\
\text { Evaluation Plan }\end{array}$ & $\begin{array}{l}\text { - Describe the programme } \\
\text { - Describe programme outcomes and effect questions } \\
\text { - Write questions based on matrix } \\
\text { - Write process questions } \\
\text { - Develop indicators and measures } \\
\text { - Specify evaluation designs }\end{array}$ \\
\hline
\end{tabular}

Implementation

1Derived from Prins and colleagues (Prins, van Empelen, Beenackers, Brug, \& Oenema, 2010)

In line with the IM protocol, the present dissertation can be divided into two parts: 1) the needs assessment carried out to explain overweight-related behaviors (i.e., Chapters 2-4), and 2) the intervention development and evaluation (i.e., Chapters 5-6). 


\section{PART I: The Needs Assessment}

It is generally assumed that overweight is caused by a positive energy balance resulting from increased dietary intake (DI) and inadequate physical activity (PA) (Slater et al., 2009; Swinburn et al., 1998). For decades, differences in DI and PA have been explained by cognitive models, in which behavior is mainly explained in terms of behavioral intentions.

\section{Cognitive Determinants of Overweight-Related Behaviors}

One globally accepted social-cognitive model is the Theory of Planned Behavior (TPB) proposed by Ajzen (1991). The TPB model states that actual behavior is guided by intentions and perceived behavioral control (PBC). However, whilst this model has been applied in many studies, it has been shown that only about $20 \%$ of behavior can be explained by intentions (the most proximal determinant of behavior), and these intentions account for only about 12\% of actual PA among youth (McEachan, Conner, Taylor, \& Lawton, 2011; Motl et al., 2002). Moreover, as Conner et al. (2013) have indicated that associations between intentions and behavior are even weaker among low SES groups, it is therefore important to search for other factors that may explain overweight-related behaviors (Conner et al., 2013).

\section{Alternative Motivational and Contextual Factors Determining Overweight-Related Behaviors}

One possible explanation for the "intention-behavior gap" may be found in socioecological models. These models suggest that health behavior is the result of an interplay between motivational and contextual factors (Kremers et al., 2006; Sallis, Johnson, Calfas, Caparosa, \& Nichols, 1997; Spence \& Lee, 2003). Motivational factors that may stimulate DI and PA include affect (mood) and the perceived environment, (the perceived availability of sports facilities) (Carels et al., 2001; Magnan, Kwan, \& Bryan, 2013; Penedo \& Dahn, 2005; Prins, Oenema, van der Horst, \& Brug, 2009). For example, the perceived availability of sports facilities has been associated with compliance with the Dutch "fitnorm" (this norm recommends that individuals participate in vigorous PA at least three times per week) and the perceived availability of parks has been associated with long-term increases in walking and cycling (Prins, Oenema, van der Horst, \& Brug, 2009). Contextual factors reflect the physical environment, the social environment, as well as the availability of food and the opportunities for participation in PA (Kremers et al., 2006). Although explanatory studies on DI and PA provide valuable insights to the field, their findings mainly rely on self-reported data and are, therefore, vulnerable to methodological issues (Stone, Shiffman, \& De Vries, 2000). 


\section{Possible Methodological Issues in Monitoring Overweight-Related Behaviors}

Methodological issues that occur during retrospective reporting on complex behaviors (e.g., overweight-related behaviors) include, for example, recall bias and social desirability effects (Stone, Shiffman, \& De Vries, 2000). Problems with recall are more likely to occur with regard to behaviors that are frequent and irregular, are intensive, that change over time, and that have to be reflected upon within the specific context in which the original behavior was performed (Stone, Shiffman, Atienza, \& Nebeling, 2007). In recent years, and in reaction to these methodological issues, diary studies have become more popular as they tend to limit the recall period to 24 hours. However, this method is still subject to poor or faked compliance and erroneous data (e.g., missing data and ambiguous responses) (Broderick et al., 2003; Stone et al., 2007). A promising method that can be used to overcome these issues is mobile-based Ecological Momentary Assessment; mEMA.

\section{Mobile-based Ecological Momentary Assessment}

Although Ecological Momentary Assessment (EMA) may sound fancy, the EMA framework encompasses multiple preexisting methods that are well-known and based on real-time, real-world data collection, such as behavioral observations and diary studies (Stone, Shiffman, Atienza, \& Nebeling, 2007). The promise of this type of data collection is that it focuses on the collection of concurrent rather than retrospective self-reports, eliminating the memory-related biases that are inherent to retrospective reports. A second core benefit of the EMA approach is that ecological validity is realized by collecting data in real world settings (Stone et al., 2007). Bearing in mind these methodological advantages, it is highly likely that EMA is a suitable method with which to monitor complex behaviors that fluctuate over time and are vulnerable to environmental influences, such as DI and PA.

Fundamentally, EMA relies on participants to be compliant with the data collection protocol (Stone et al., 2007). Although compliance rates in many diary studies appear at first glance to be sufficient, subsequent studies have shown that participants routinely fail to complete their diary entries in a timely way and therefore start hoarding and backfilling diary entries, also known as "parking lot compliance" (Stone et al., 2007). Fortunately, in line with technological development, EMA research has moved on from traditional paper and pencil diaries, to electronic EMA (eEMA) and mobile-based EMA (mEMA), which allow for participant prompting, sending out reminders, and the objective registration of diary entries. Additionally, as mEMA apps are downloaded on participants' mobile phones, which are carried around most of the time, compliance with the study is expected to be even higher 
as compared to eEMA (generally based on personal digital assistants (PDA)). mEMA also allows for more objective registration of data, such as GPS data and sensory data, creating a complementary assessment tool for studying complex health behaviors such as DI and PA. As such, this needs assessment can function perfectly well as a first step in systematic intervention development.

\section{Part II: The Intervention}

\section{Internet-Delivered Interventions to Change Overweight-Related Behaviors}

Over the past few years, the number of internet-delivered interventions targeting health behavior change has grown rapidly. These interventions hold great promise for health promotion. Several meta-analyses have highlighted the major advantages of online interventions (e.g., high reach, low costs, and convenience for its users), and pointed to their effectiveness (Crutzen, Kohl, \& De Vries, 2013; Griffiths, Lindenmeyer, Powell, Lowe, \& Thorogood, 2006). However, these studies also show that the effectiveness of interventions varies considerably (Krebs, Prochaska, \& Rossi, 2010; Kroeze, Werkman, \& Brug, 2006; Verweij, Coffeng, van Mechelen, \& Proper, 2011; Webb, Joseph, Yardley, \& Michie, 2010). One possible explanation for the variability in effect sizes concerns the behavior change methods that have been applied in the different studies. Webb et al. used a taxonomy of effective methods, also called behavior change techniques (BCT), by Abraham and Michie who identified 26 BCTs that can be used to stimulate behavior change (Abraham \& Michie, 2008; Webb et al., 2010). Results showed that the inclusion of multiple BCTs resulted in higher intervention effectiveness. In line with these findings, Dusseldorp et al. developed and applied a Meta-CART (Classification And Regression Trees) method to identify effective combinations of BCTs (Dusseldorp, van Genugten, van Buuren, Verheijden, \& van Empelen, 2013). They found that providing information about the link between behavior and health combined with the prompting of intention formation resulted in high effect sizes in PA and DI studies, as did the combination of providing information about consequences and the use of follow-up prompts. A review by Krebs, Prochaska, and Rossi (2010) focused solely on the outcome measures of interventions targeting dietary fat reduction, fruit \& vegetable intake, and physical activity and found support for the feasibility of targeting multiple behaviors simultaneously (Krebs et al., 2010; Vandelanotte, Reeves, Brug, \& De Bourdeaudhuij, 2008). Based on the findings of Webb et al., Dusseldorp et al., and Krebs et al., we can conclude that the simultaneous inclusion of multiple BCTs and outcome measures in interventions targeting DI and PA will be optimal. 


\section{Challenges of Internet-Delivered Interventions}

Although internet-delivered interventions (or so-called e-Health interventions) seem promising in targeting DI and PA behaviors, many of these e-Health studies report low study compliance and high drop-out rates, sometimes resulting in low or even absent intervention effects (Crutzen et al., 2013; van Genugten, 2011). Alongside the effectiveness of an intervention (i.e., internal validity), it is therefore of great importance to focus on the reach and use of the intervention by the target group (Bennett \& Glasgow, 2009). One framework that takes into account factors that contribute to the public health impact of an intervention is the RE-AIM framework, representing the Reach, Effectiveness, Adoption, Implementation and Maintenance of an intervention (Glasgow, Vogt, \& Boles, 1999). This RE-AIM framework was used as a starting point in a recent review by Crutzen et al. (Crutzen et al., 2013). Crutzen et al. indicated that while e-Health interventions potentially have a large reach, these internet-delivered interventions mostly reach a particular demographic - i.e. the female, Caucasian, highly educated part of the population. These individuals are not necessarily attracted at the same rate and by the same intervention features as high-risk participants such as youth from low socioeconomic families and minority groups (Crutzen et al., 2013; Glasgow et al., 2007). As such, e-Health interventions often struggle with low actual use and user engagement (Crutzen et al., 2013).

\section{Serious Games Used to Optimize Behavior Change Processes and Intervention Use}

A promising method designed to be both educational and engaging is serious gaming (DeSmet et al., 2014). Serious games are computer- or smartphone-delivered interventions which target healthrelated behaviors while being enjoyable, attention-captivating and intrinsically motivating (DeSmet et al., 2014; Prensky, 2007). These motivational aspects are thought to increase learning effects through: 1) immersion (i.e., absorption in game play (and suspension of disbelief), while creating personally relevant experiences and deep affection for the characters, 2) game flow (i.e., the balance between skills and challenge), and 3) meeting the individual's needs for mastery, autonomy, and relatedness (i.e., as outlined in self-determination theory; SDT) (DeSmet et al., 2014; Ryan \& Deci, 2000). Moreover, these smartphone-delivered serious games have the potential to integrate behavior change techniques in an efficient way. For example, bearing in mind that the majority of Dutch youth carry around their smartphone $24 / 7$, prompting them with reminders about their goals and to play the game may potentially improve intervention use as prompting intention formation has been indicated as an effective behavior change technique (Abraham \& Michie, 2008). 


\section{Aims and Outline of This Thesis}

The aims of this thesis are as follows: 1) to gain a better understanding of secondary vocational education students' overweight-related behaviors (i.e., dietary intake [DI] and physical activity [PA]) in real-time settings, 2) to examine whether serious gaming can change these behaviors, and 3) to investigate the added value of Intervention Mapping (IM) underlying the development of serious game interventions. These main objectives are discussed in the following chapters, which are structured according to the different steps in Intervention Mapping (IM). Chapter $\mathbf{2}$ describes the feasibility and usability of mEMA. Chapter 3 describes the results from a mobile-based Ecological Momentary Assessment (mEMA) study regarding the role of motivational and contextual factors on planned and impulsive snack consumption. Chapter 4 describes a similar mEMA study regarding the influence of motivational and contextual factors on physical activity behaviors among emerging young adults, and specifies both the multivariate as well as the time lagged model. These two chapters account for the first step in IM, the needs assessment. Chapter $\mathbf{5}$ describes the design rationale behind the serious self-regulation game intervention "Balance it", which aims to prevent secondary vocational education students in the Netherlands from gaining weight (IM step 2-5). Next, we evaluated the intervention effects on students' DI and PA behaviors and intervention use in Chapter 6. Finally, this thesis is completed with a general discussion in Chapter 7. 


\section{References}

Abraham, C., \& Michie, S. (2008). A taxonomy of behavior change techniques used in interventions. Health Psychology, 27(3), 379-387.

Ajzen, I. (1991). The theory of planned behavior. Organizational Behavior and Human Decision Processes, 50, 179-211.

AMA adopts new policies on second day of voting at annual meeting 2013. (2013). Retrieved from http://www.ama-assn.org/ama/pub/news/news/2013/2013-06-18-new-ama-policies-annualmeeting.page

Bartholomew, L. K., Parcel, G. S., Kok, G., Gottlieb, N. H., \& Fernández, M. E. (Eds.). (2011). Planning health promotion programs: An intervention mapping approach. San Francisco: Jossey-Bass.

Bennett, G. G., \& Glasgow, R. E. (2009). The delivery of public health interventions via the internet: Actualizing their potential. Annual Review of Public Health, 30, 273-292.

Broderick, J. E., Schwartz, J. E., Shiffman, S., Hufford, M. R., \& Stone, A. A. (2003). Signaling does not adequately improve diary compliance. Annals of Behavioral Medicine, 26, 139-148.

Carels, R. A., Hoffman, J., Collins, A., Raber, A. C., Cacciapaglia, H., \& O’Brien, W. H. (2001). Ecological momentary assessment of temptation and lapse in dieting. Eating Behaviors, 2, 307-321.

Conner, M., McEachan, R., Jackson, C., McMillan, B., Woolridge, M., \& Lawton, R. (2013). Moderating effect of socioeconomic status on the relationship between health cognitions and behaviors. Annals Behavioral Medicine, 46, '19-30.

Crutzen, R., Kohl, L., \& De Vries, N. K. (2013). Kennissynthese online preventie

DeSmet, A., van Ryckeghem, D., Compernolle, S., Baranowski, T., Thompson, D., Crombez, G., ... De Bourdeaudhuij, I. (2014). A meta-analysis of serious digital games for healthy lifestyle promotion. Preventive Medicine, 69, 95-107.

DUO (2013). Aantallen 2012-2013. Retrieved from https://duo.nl/organisatie/pers/aantallen.asp 
Dusseldorp, E., van Genugten, L., van Buuren, S., Verheijden, M. W., \& van Empelen, P. (2013). Combinations of techniques that effectively change health behavior: Evidence from meta-analysis. Health Psychology,

Glasgow, R. E., Nelson, C. C., Kearney, K. A., Reid, R., Ritzwoller, D. P., Strecher, V. J., . . Wildenhaus, K. (2007). Reach, engagement, and retention in an internet-based weight loss program in a multi-site randomized controlled trial. Journal of Medical Internet Research, 9, e11.

Glasgow, R. E., Vogt, T. M., \& Boles, S. M. (1999). Evaluating the public health impact of health promotion interventions: The RE-AIM framework. American Journal of Public Health, 89(9), 1322-1327.

Griffiths, F., Lindenmeyer, A., Powell, J., Lowe, P., \& Thorogood, M. (2006). Why are health care interventions delivered over the internet? A systematic review of the published literature. Journal of Medical Internet Research, 8(2), e10.

Kerncijfers 2007-2011. (2012). Retrieved from http://www.rijksoverheid.nl/documenten-en-publicaties/jaarverslagen/2012/05/16/kerncijfers-2007-2011.html

Krebs, P., Prochaska, J. O., \& Rossi, J. S. (2010). A meta-analysis of computer-tailored interventions for health behavior change. Preventive Medicine, 51, 214-221.

Kremers, S. P. J., De Bruin, G., Visscher, T. L. S., van Mechelen, W., De Vries, N., \& Brug, J. (2006). Environmental influences on energy balance-related behaviors: A dual-process view. International Journal of Behavioral Nutrition and Physical Activity, 3(9).

Kroeze, W., Werkman, A., \& Brug, J. (2006). A systematic review of randomized trials on the effectiveness of computer-tailored education on physical activity and dietary behaviors. Annals of Behavioral Medicine, 31(3), 205-223.

Maffeis, C., \& Tato, L. (2001). Long-term effects of childhood obesity on morbidity and mortality. Hormone Research, 55, 42-45.

Magnan, R. E., Kwan, B. M., \& Bryan, A. D. (2013). Effects of current physical activity on affective response to exercise: Physical and social-cognitive mechanisms. Psychology \& Health, 28(4), 418-433.

MBO Raad (2014). Mbo-scholen. Retrieved from http://www.mboraad.nl/?category/424202/Mbo+in+feiten+en+cijfers.aspx 
McEachan, R. R. C., Conner, M., Taylor, N., \& Lawton, R. J. (2011). Prospective prediction of healthrelated behaviors with the theory of planned behavior: A meta-analysis. Health Psychology Review, $5,97-144$.

Motl, R. W., Dishman, R. K., Saunders, R., Dowda, M., Felton, G., Ward, D. S., \& Pate, R. R. (2002). Examining social-cognitive determinants of intention and physical activity among black and white adolescent girls using structural equation modeling. Health Psychology, 21, 459-467.

Must, A. (2003). Does overweight in childhood have an impact on adult health? Nutrition Reviews, 61, 139-142.

Penedo, F. J., \& Dahn, J. R. (2005). Exercise and well-being: A review of mental and physical health benefits associated with physical activity. Current Opinion in Psychiatry, 18(2), 189-193.

Polder, J. J., \& Takkern, J. (2002). Kosten van ziekten in nederland - de zorgeuro ontrafeld [cost of illness in the netherlands]. (No. 304). Bilthoven: RIVM.

Prensky, M. (2007). Digital game-based learning. St. Paul, Minnesota: Paragon House.

Prins, R. G., van Empelen, P., Beenackers, M. A., Brug, J., \& Oenema, A. (2010). Systematic development of the YouRAction program, a computer-tailored physical activity promotion intervention for dutch adolescents, targeting personal motivations and environmental opportunities. BMC Public Health, 10, 474 .

Prins, R. G., Oenema, A., van der Horst, K., \& Brug, J. (2009). Objective and perceived availability of physical activity opportunities: Differences in associations with physical activity behavior among urban adolescents. International Journal of Behavioral Nutrition and Physical Activity, 6, 70.

Ronda, G., van Assema, P., \& Brug, J. (2001). Stages of change, psychological factors and awareness of physical activity levels in the netherlands. Health Promotion International, 16(4), 305-314.

Rubenstein, A. H. (2005). Obesity: A modern epidemic. Transactions of the American Clinical and Climatological Association, 116, 103-113.

Ryan, R. M., \& Deci, E. L. (2000). Intrinsic and extrinsic motivations: Classic definitions and new directions. Contemporary Educational Psychology, 25(1), 54-67. 
Sallis, J. F., Johnson, M., Calfas, K., Caparosa, S., \& Nichols, J. (1997). Assessing the perceived physical environmental variables that may influence physical activity. Research Quarterly for Exercise and Sport, 68, 345-351.

Seidell, J. C. (2000). The current epidemic of obesity. In C. (. ). Bouchard (Ed.), Physical activity and obesity (2nd ed., pp. 21-30). Champaign, IL: Human Kinetics.

Schönbeck, Y., \& van Buuren, S. (2010). Factsheet resultaten vijfde landelijke groeistudie. TNO Leiden.

Slater, J., Green, C. G., Sevenhuysen, G., Edginton, B., O’Neil, J., \& Heasman, M. (2009). The growing canadian energy gap: More the can than the couch? Public Health Nutrition, 12(11), 2216-2224.

Spence, J. C., \& Lee, R. E. (2003). Toward a comprehensive model of physical activity. Psychology of Sport and Exercise, 4, 7-24.

Stone, A. A., Shiffman, S., Atienza, A. A., \& Nebeling, L. (2007). The science of real-time data capture. self-reports in health research. New York: Oxford University Press.

Stone, A. A., Shiffman, S., \& de Vries, M. W. (2000). Ecological momentary assessment. In D. Kahneman, E. Diener \& N. Schwarz (Eds.), Well-being: The foundations of hedonic psychology. (pp. 26-39). New York: Russell Sage Foundation.

Swinburn, B. A., Sacks, G., Lo, S. K., Westerterp, K. R., Rush, E. C., Rosenbaum, M., .. Ravussin, E. (1998). Estimating the changes in energy flux that characterize the rise in obesity prevalence. American Journal of Clinical Nutrition, 89(6), 1723-1728.

European Heart Initiative (2001). Children and young people - the importance of physical activity. Retrieved from http://ec.europa.eu/health/ph_projects/2000/promotion/fp_promotion_2000_ frep_11_en.pdf

Van Genugten, L. (2011). Prevention of weight gain among adults: Development and evaluation of a computer-tailored self-regulation intervention.

Vandelanotte, C., Reeves, M. M., Brug, J., \& de Bourdeaudhuij, I. (2008). A randomized trial of sequential and simultaneous multiple behavior change interventions for physical activity and fat intake. Preventive Medicine, 46, 232-237. 
Verweij, L. M., Coffeng, J., van Mechelen, W., \& Proper, K. I. (2011). Meta-analyses of workplace physical activity and dietary behaviour interventions on weight outcomes. Obesity Reviews, 12, 406-429.

Wang, Y., Liang, H., Tussing, L., Braunschweig, C., Cabellero, B., \& Flay, B. (2007). Obesity and related risk factors among low socio-economic status minority students in chicago. Public Health Nutrition, 10(9), 927-938.

Wang, Y., Monteiro, C., \& Popkin, B. M. (2002). Trends of obesity and underweight in older children and adolescents in the united states, brazil, china, and russia. The American Journal of Clinical Nutrition, 75, 971-977.

Wang, Y. C., McPherson, K., Marsh, T., Gortmaker, S. L., \& Brown, M. (2011). Health and economic burden of the projected obesity trends in the USA and the UK. Lancet, 378, 815-825.

Webb, T., Joseph, J., Yardley, L., \& Michie, S. (2010). Using the internet to promote health behavior change: A systematic review and meta-analysis of the impact of theoretical basis, use of behavior change techniques, and mode of delivery on efficacy. Journal of Medical Internet Research, 17(12), e4.

World Health Organization [WHO] (2013a). Obesity and overweight. (Fact sheet No. 311).WHO. Retrieved from http://www.who.int/mediacentre/factsheets/fs311/en/

World Health Organization [WHO] (2013b). Nutrition, physical activity and obesity: Netherlands. Retrieved from http://www.euro.who.int/_data/assets/pdf_file/0018/243315/ Netherlands-WHO-Country-Profile.pdf 



\section{CHAPTER 2}

Feasibility, Usability, and Ecological Validity of a mobile-based

Ecological Momentary Assessment (mEMA) Tool: Monitoring Dietary

Intake and Physical Activity Electronically

Published: Spook, J.E., Paulussen, T., Kok, G., \& van Empelen,

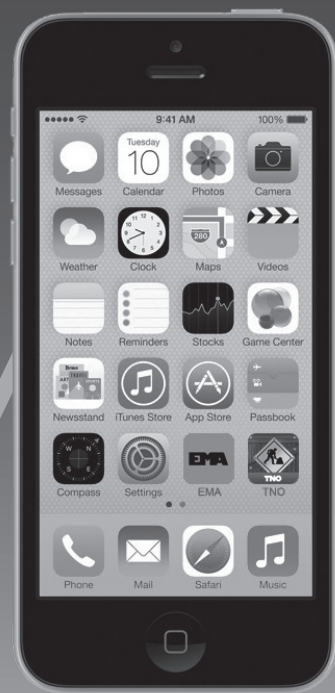

P. (2013). Monitoring dietary intake and physical activity

electronically: feasibility, usability, and sensitivity of a mobilebased Ecological Momentary Assessment (mEMA) tool. Journal

of Medical Internet Research, 15(9), e214. 


\title{
Feasibility, Usability, and Ecological Validity of a mobile-based Ecological Momentary Assessment (mEMA) Tool: Monitoring Dietary Intake and Physical Activity Electronically
}

\begin{abstract}
Background: Despite the growing body of research on complex lifestyle behaviors (e.g., Dietary Intake [DI] and Physical Activity [PA]), monitoring of these behaviors has been hampered by suitable methods. A possible solution to this deficiency is mobile-based Ecological Momentary Assessment ( $m E M A)$, which enables researchers to collect data on participants' states in real-time by means of a smartphone application. However, feasibility, usability, and ecological validity need to be anticipated on and managed in order to enhance the validity of mEMA. Objective: Examining feasibility, usability and ecological validity of a mEMA-application (app) with regard to DI and PA among Dutch vocational education students. Methods: The students $(N=30)$ participated in the mEMA-study for seven consecutive days. They downloaded the mEMA-app on their smartphone. Feasibility and usability of the mEMA-app were evaluated by completing an online evaluation after seven days of participation. Ecological validity was measured by assessing the degree to which the content of the mEMA-app approximates the real-world setting that is being examined, containing several multiple choice questions. Results: Compliance rates, as registered by the mEMA-app, declined $46 \%$ over a seven-day period, while self-reported compliance, as measured with an online evaluation questionnaire afterwards, indicate a smaller decrease in compliance (29\%). The students evaluated the mEMA-app as feasible and usable. Ecological validity analyses showed that all DI and almost all PA multiple choice options were covered with the compound response categories. Conclusion: the mEMA-app offers the opportunity for assessing complex health behaviors (e.g., DI \& PA) in real-time settings, in which specifically routinized behaviors are involved. However, the mEMA-app faced several challenges that needed to be overcome in order to improve its validity. As such, the present study showed that the mEMA-app is a usable and ecologically valid tool to measure DI and PA behaviors among secondary vocational education students, but compliance is still limited.
\end{abstract}

Keywords: mobile-based Ecological Momentary Assessment (mEMA), Smartphone Application, Feasibility, Usability, Ecological validity, Dietary Intake, Physical Activity 


\section{Introduction}

Self-report of lifestyle behaviors, affect, and cognitions is often biased (e.g., recall bias, availability, and recency) (Bogers, Brug, Van Assema van, \& Dagnelie, 2004; Haedt-Matt \& Keel, 2011; Shiffman, Stone, \& Hufford, 2008; Stone, Shiffman, Atienza, \& Nebeling, 2007; Stone \& Shiffman, 2002). For instance, studies of fruit and vegetable consumption and their underlying determinants have shown that people generally overestimate their own consumption and, as such, behavior is less well predicted among those with an optimistic bias (Lechner, Brug, \& de Vries, 1997). Similar findings have been observed for fat consumption and physical activity (Brug \& van Assema van, 2001; Ronda, van Assema, \& Brug, 2001). Similarly, Nordgren et al (2008) showed in a study targeting smoking behavior that health cognitions are unstable and dynamic (Nordgren, van der Pligt, \& van Harreveld, 2008).

Hence, it is important to find ways to more reliably assess determinants and behavior, in order to better understand and change behaviors. A method that could reduce the threat of recall bias, availability, and recency effects is ambulatory monitoring, such as Ecological Momentary Assessment (EMA) (Stone \& Shiffman, 1994). As Stone, Shiffman, Atienza, and Nebeling state: “The core rationale for EMA methods rests on three core benefits of the EMA approach: (1) avoidance of recall and its attendant bias, by collecting data on momentary states; (2) realization of ecological validity by collecting data in the real world; and (3) achievement of temporal resolution, enabling analysis of dynamic processes over time" (p.6, (Stone et al., 2007)). As such, EMA enables assessment of complex health behaviors and related factors of influence in real-time settings (Serre et al., 2012), and appears to be applicable to often routinized behaviors (Stone \& Shiffman, 2002). In general, a comparison of EMA with traditional recall-based methodologies has shown that EMA produces more reliable results (Shiffman et al., 2008).

With the increasing popularity of smartphones, recent studies have indicated the usefulness of mobile-based Ecological Momentary Assessment (mEMA) (Collins, Kashdan, \& Gollnisch, 2003; Daugherty et al., 2012; Heinonen, Luoto, Lindfors, \& Nygard, 2012; Ozdalga, Ozdalga, \& Ahuja, 2012; Rofey et al., 2010; Schnall et al., 2013; Tsai et al., 2007; Zhang et al., 2012). The advantage of mEMA is that it is incorporated in a tool that is frequently used in daily living. However, researchers have also suggested that mEMA faces challenges that need to be anticipated and managed in order to enhance its validity (e.g., time of day, day of the week, concurrent activities or states, nonresponse, and missing diary entries (Stone et al., 2007). As such, it is essential to achieve adequate compliance 
and to study the feasibility, usability, and ecological validity of mEMA and mEMA measurements. Within this study, feasibility and usability refer to the practical application of mEMA in daily life (e.g., monitoring burden). Ecological validity refers to the extent to which the data are representative of the possible range of experiences in daily life (Stone et al., 2007) and we explicitly focused on the extent to which we included the range of most relevant social and physical environments.

To date, use of mEMA in health promotion research is limited, especially when focusing on weight-related factors (i.e., Dietary Intake [DI] and Physical Activity [PA]). To ensure that mEMA can be used effectively to monitor determinants of DI and PA in future research, it is imperative that these challenges are examined prior to its actual deployment (Mays et al., 2010). To examine mEMA, the present study examined three research questions concerning the validity of a mEMA app: Is the mEMA app (1) a feasible (i.e., what is the level of students' compliance), (2) usable (i.e., how is mEMA evaluated by the students), and (3) ecologically valid (i.e., do the social and physical response items capture the most important day-to-day social and physical characteristics (Stone et al., 2007) tool to measure determinants of DI and PA among secondary vocational education students.

\section{Methods}

\section{Participants}

Data were collected from three secondary vocational education schools in the Netherlands. Out of the 44 students who were approached to participate in the mEMA study, 30/44 students (68\%) participated for 7 consecutive days (17/30, 57\% were female), of which 17 students (aged 16-21 years) completed the online evaluation (regarding feasibility and usability of the mEMA app) after their 7 days of participation. Participatory incentives of ten $€ 20$ coupons were randomly distributed to the students at the end of the study. Those who participated for 7 days and filled in the online evaluation form doubled their chance of winning. All students consented to take part in the study and students aged $\leq 17$ were provided with a passive consent form for their parents to complete. All procedures were approved by The Ethical Committee of Psychology (Maastricht University).

\section{Measures and Procedures}

In a study conducted prior to the mEMA study ( $N=305$ secondary vocational education students), an assessment was made of the type of smartphone platforms that were most commonly used among 
the secondary vocational education students. The majority of the students $(244 / 305,79.9 \%)$ indicated that they used a smartphone operating on BlackBerry OS, Android, or iOS (respectively 151/305, $49.5 \%, 55 / 305,18.1 \%$, and $37 / 305,12.1 \%)$. Estimating an increased use of the iOS and Android platforms, the mEMA app was built on all three platforms.

\section{Mobile Ecological Momentary Assessment (mEMA) Study}

In total, 44 students from three different secondary vocational education schools in the Netherlands were approached by their teachers to participate in the present study, of which 30 students participated in the mEMA study. Students from the first two schools were allocated to the first group of participants ( $N=14)$ and were randomly assigned to the DI or PA condition (study design and participant distribution are illustrated in Figure 1). The students from the third school were allocated to the second group ( $N=16)$ and started using the mEMA app after several adjustments were made based on feedback from the first group (discussed in the Results section). Participants in the second group were encouraged to use the mEMA app similarly to those participating in the first group.

Figure 1: Study design and participant distribution

\begin{tabular}{|c|c|c|c|c|}
\hline \multirow{3}{*}{$\begin{array}{l}\text { Phase 1: Inventory } \\
\qquad N=305\end{array}$} & \multicolumn{4}{|c|}{$\begin{array}{l}\text { Phase 2: mEMA study } \\
\qquad N=30\end{array}$} \\
\hline & \multicolumn{2}{|c|}{$\begin{array}{l}\text { Group 1: } \\
\qquad N=15\end{array}$} & \multicolumn{2}{|c|}{$\begin{array}{l}\text { Group 2: } \\
\qquad N=15\end{array}$} \\
\hline & $\begin{array}{l}\text { Evaluation: } \\
\qquad N=11\end{array}$ & $\begin{array}{l}\text { No Evaluation: } \\
\qquad N=4\end{array}$ & $\begin{array}{l}\text { Evaluation: } \\
\qquad N=6\end{array}$ & $\begin{array}{l}\text { No Evaluation: } \\
\qquad N=9\end{array}$ \\
\hline
\end{tabular}

\section{Mobile Ecological Momentary Assessment App Use}

Prior to the use of the mEMA app, students completed an online questionnaire regarding their DI or PA during the preceding 7 days. Data from this online questionnaire were collected for other study purposes. After completion, students who possessed a smartphone that operated on iOS (iPhone version 5.0), BlackBerry OS (version 6.0 or 7.0), or Android (version 2.2 and over) systems were eligible for participation, and downloaded the mEMA app from the BlackBerry OS, iOS, or Android app store 
$(N=30)$. Students who possessed a smartphone with other operating systems or who did not own a smartphone were exempted from further participation. The students were invited to use the mEMA app for 7 consecutive days and gave their consent for participation. During these 7 days, participants were asked to fill in the same short questionnaires regarding their DI or PA during the preceding 3.5 hours, 5 times a day. Participants were able to close the app at any time.

\section{Evaluation}

All students who participated in the mEMA study were asked to fill in an online evaluation form concerning the feasibility and usability (i.e., functionality and interface) of the mEMA app. Additionally, after participants from the first group filled in the online evaluation form, group discussions took place per school, led by one of the researchers.

\section{Content Management System (CMS)}

In order to upload content for the online questionnaire, mEMA app (both DI and PA conditions), and the evaluation, a content management system (CMS) was built. This CMS enabled researchers to monitor multiple complex health behaviors simultaneously (e.g., DI and PA) and to adapt the content, text, and prompting sequence.

\section{Implementation Procedure for Using the mEMA App}

An interval-contingent schedule was used for the mEMA app, which initially prompted participants at 5 time periods per day $(8: 00 \mathrm{am}, 12: 00 \mathrm{pm}, 3: 30 \mathrm{pm}, 6: 30 \mathrm{pm}$, and 9:30pm with a range of 30 minutes each), tailored to the schedule of their schools. These prompts were formatted as auditory signals and were displayed on their smartphone screens. When a diary entry was missed or rejected, up to two reminder signals were sent, once after 30 minutes and the second after 60 minutes. After missing and/or rejecting the two reminders, the CMS noted the initial diary entry as "missed".

First, participants received instructions about the study and the use of the mEMA app (i.e., how to download and use the app). They were instructed to start with an online questionnaire regarding their DI or PA during the preceding week. Second, students were able to use the app during the first day to get familiar with it. From the second day, the mEMA app started prompting participants, asking them to fill in a short questionnaire. Students were instructed to respond to these prompts as much as possible. 
During each diary prompting sequence, students who were allocated to the DI condition received a total of 14-16 questions regarding their mood, eating behavior, food cravings, self-evaluative emotions, location, activities, social context, soft and energy drinks, snack intention, hunger, and the availability of food, respectively (i.e., 12-14 questions depending on their responses within the decision tree; see Figure $2 \mathrm{~A}$ ). These questions were derived from Carels et al, White et al, Dijkstra and Buunk, Adriaanse et al, Thomas, Thomas et al, and Rijpstra et al. (Adriaanse, Ridder de, \& Wit de, 2009; Carels et al., 2001; Dijkstra \& Buunk, 2008; Thomas, 2009; Thomas, Doshi, Crosby, \& Lowe, 2011; White, Whisenhunt, Williamson, Greenway, \& Netemeyer, 2002). Students in the PA condition received 12-14 questions concerning their mood, sedentary behavior, physical activity, need for physical activity, evaluative emotions, location, social context, behavior intention, active transport, possible barriers, and feelings of security, respectively (see Figure 2 B). The PA questionnaires were derived from Carels et al, Cranford et al, de Vries et al, Dunton et al, Dunton et al, Grow et al, and Prins et al. (Carels et al., 2001; Cranford et al., 2006; de Vries, Bakker, van Mechelen, \& Hopman-Rock, 2007; Dunton, Whalen, Jamner, \& Floro, 2007; Dunton, Liao, Intille, Spruijt-Metz, \& Pentz, 2011; Grow et al., 2008; Prins, Empelen van, Beenackers, Brug, \& Oenema, 2010). 
Figure 2: Decision Trees of the MEMA app DI and PA Questionnaire

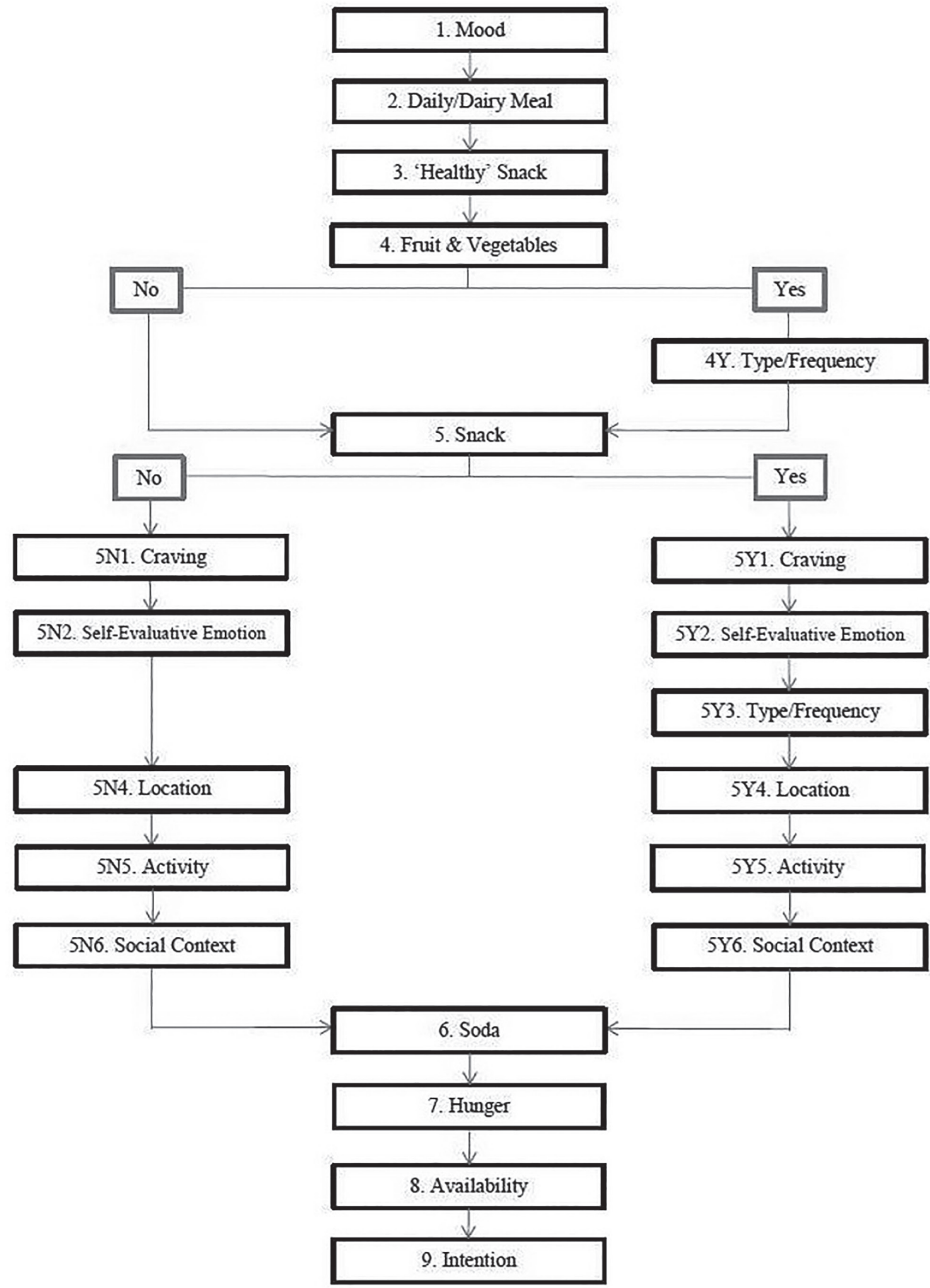




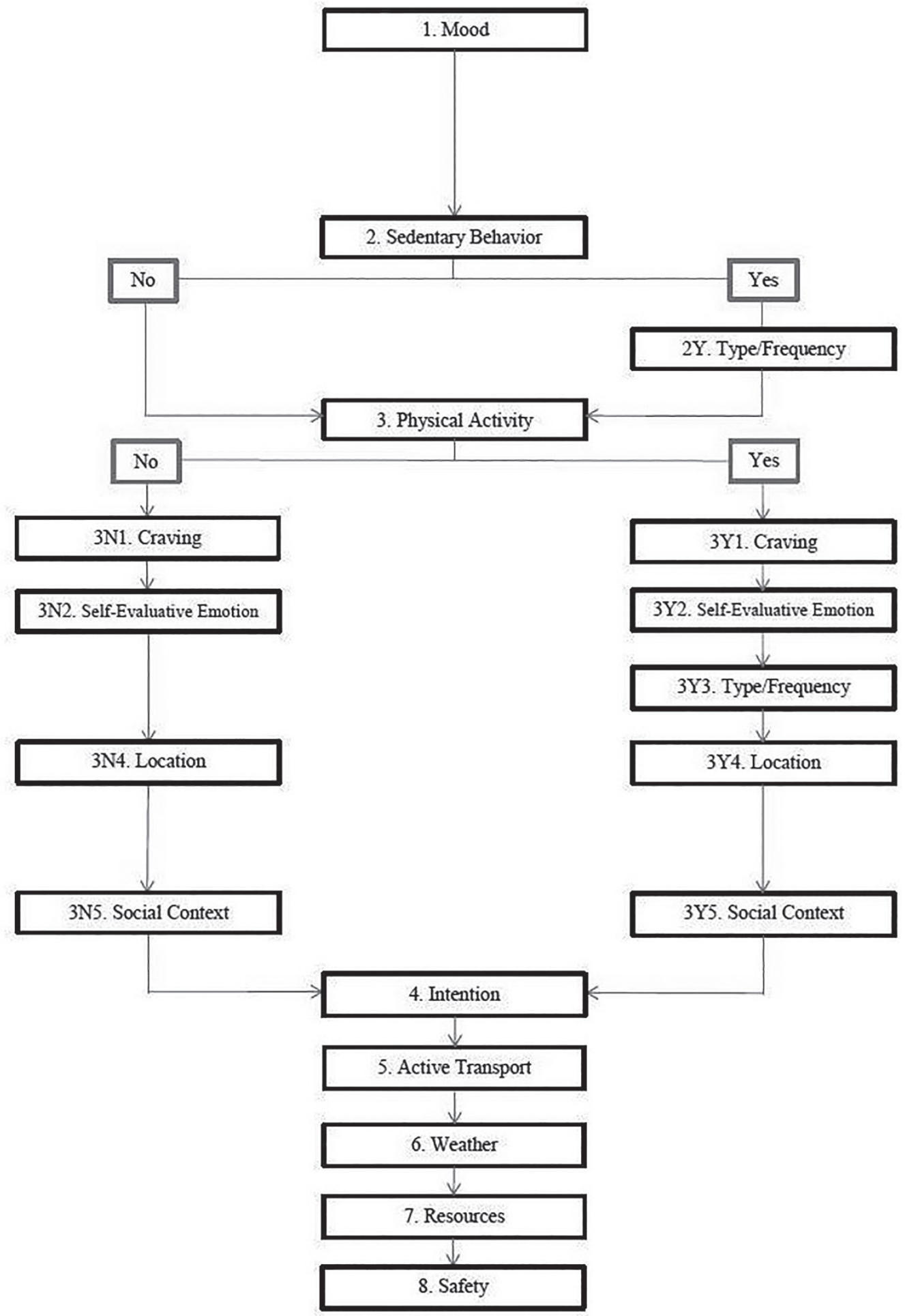


Four different types of response categories were used: Visual Analogue Scale (VAS slider) (see Figure 3 A), multiple-choice bullets (see Figure 3 B), multiple-choice open field combined with VAS slider (see Figure $3 \mathrm{C}$ ), and binary (see Figure $3 \mathrm{D}$ ). In the DI condition, 5-point (VAS slider) scales were used to measure mood, hunger, availability, and intention (e.g., mood: “At the moment, I'm feeling happy"). Multiple-choice bullets were used as response categories for daily meal, self-evaluative emotions, location, activity, and social context (e.g., location: "Where were you when you were having a snack?"). Multiple-choice open field combined with VAS sliders were used to indicate the type and frequency of fruits and vegetables, snacks, and sodas they had consumed (e.g., "In the last three and a half hours, what type of snack(s) did you eat? And how many?"). These sliders ranged from 0-100, but the open fields enabled participants to fill in amounts that were larger than 100. Finally, binary response categories were used for decisions within the tree and food craving (e.g., "In the last three and a half hours, have you eaten fruit or vegetables?"). All examples are translated from Dutch to English.

Figure 3: Screenshots of $M E M A$

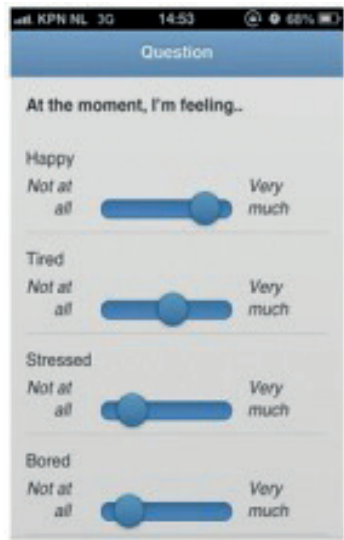

(A)

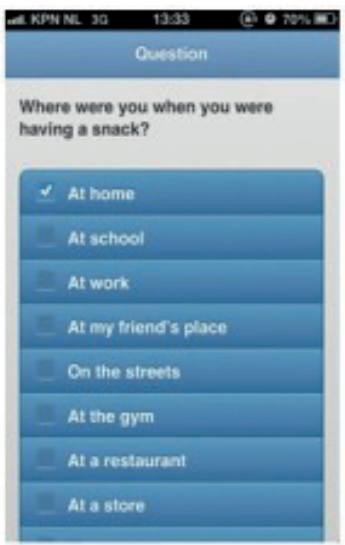

(B)

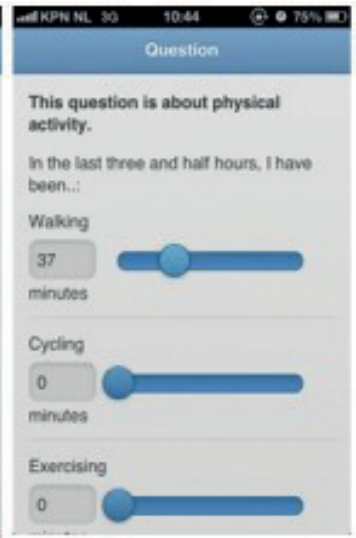

(C)

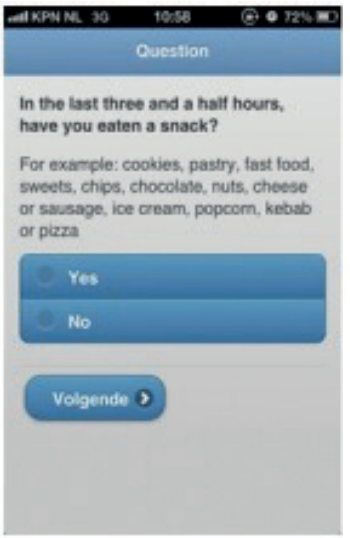

(D)

\section{Feasibility and Usability Measures}

Feasibility and usability measures were derived from previously completed studies (Heinonen et al., 2012; Tsai et al., 2007). These measures contained 17 statements about the students' subjective experience with the mEMA app (e.g., "In my opinion, it was boring to work with the mEMA app"), the level of difficulty (e.g., "In my opinion, the mEMA app was easy to use"), prompting sequence (e.g., "In my opinion, the number of prompts that were sent during one week (5 times a day) was 
annoying"), length (e.g., "In my opinion, the trial took too long"), and understanding (e.g., "In my opinion, the questions were understandable"). Responses were scaled 1 (totally disagree) to 5 (totally agree). The midpoint of the scale was considered as a criteria for further interpretation (e.g., scores above the midpoint of the usability scale were interpreted as "the mEMA app was easy to use"). Also, three open-ended questions were added to enable students to provide suggestions for further improvement of the mEMA app: (1) "Why did you ignore or postpone a prompt?", (2) "I would make the following change to the mEMA app...", and (3) "I would add the following to the mEMA app...").

\section{Ecological Validity}

Ecological validity was determined by assessing the degree to which the collected data represent the full-range of possible social and physical influences. The assessment for both DI and PA included two questions regarding the location and social context (e.g., "Where were you while having a snack?" and "While you were being physically active, how many people were there with you?"). Response categories for both the location and social context were multiple choice and similar for DI and PA. Multiple-choice options included the following locations and social settings: at home, school, work, friend's house, outside, restaurant, cafeteria, supermarket, sports club, or elsewhere; and a friend, colleague, classmate, team member, sibling, parent, partner, child, teacher, stranger, or other, respectively for the social setting.

\section{Data Analysis}

SPSS version 20 was used for data analyses. Data from the mEMA app were transported from the smartphone into a secure computer system when participants had access to a Wi-Fi connection. Next, data from the computer system were converted to an SPSS database. With SPSS, descriptive statistics were generated (means and percentages) and compliance rates were calculated for the exploration of response patterns. Furthermore, a $t$ test was done in order to examine gender differences in the perceived usability of the mEMA app. Prior to the analyses, normality was tested by means of Q-Q plots. Mean differences were analyzed using $t$ tests when normally distributed and Mann-Whitney $U$ test when they were non-normally distributed. 


\section{Results}

\section{Study Adjustments}

After the first group had participated, technical problems were fixed and both feasibility and usability were evaluated by means of the aforementioned measurement procedures and group discussions. Based on the students' feedback, two adjustments were made: (1) the number of daily prompts was reduced from 5 prompts a day to 4 prompts a day, and (2) the time sequence was reduced from 8 to 7 days of participation, as the start and evaluation of the study took place during their physical activity classes (once a week). Also, students evaluated the VAS sliders (see Figure $3 \mathrm{~A}, \mathrm{C}$ ) as bothersome. However, due to practical constraints (i.e., Likert scales did not fit within the boundaries of all smartphone screens), usage of VAS sliders was maintained. The second group made use of the same mEMA app as the first group, but they were prompted 4 times a day during 7 consecutive days, instead of 5 prompts a day during 8 consecutive days.

An independent sample $t$ test was conducted to test whether the usability of the mEMA app increased after the number of prompts was decreased from 5 to 4 times a day and the time sequence was reduced from 8 to 7 consecutive days. The results showed a significant difference between the first group $(N=11)$ and the second group $(N=6)$ regarding the app's usability $\left(t_{14}=-2.15, P=.05\right)$. The second group perceived the app as easier to use $(M=4.33, S D=.82)$ compared to the first group $(M=3.36, S D$ $=.92)$. However, no significant differences between the first group and the second group were found regarding the duration of the study $(P=.19)$ and their evaluation (i.e., how annoying) of the amount of prompting $(P=.86)$.

\section{Compliance Rate}

Students' compliance was reported per day $(N=30)$. Thirty participants started using the mEMA app at Day 1 (100\%), and 7 days later, 14 students still participated (44\%). Compliance decreased $56 \%$. Further exploration of times of day did not indicate clear differences in compliance between morning, early afternoon, late afternoon, early evening, and late evening (56\%, 55\%, 61\%, 55\%, and $56 \%$ respectively). The response patterns per time of day over 7 days of participation are projected in Figure 4. Interestingly, the mEMA app was used 23 times without being prompted (i.e., user based). The overall mean completion time per response was 138.7 seconds $(S D=65.6$; for $D I M=132.3, S D=$ 63.7] and PA $[M=146.8, S D=67.4])$. 
Total response rates were assessed by calculating the percentage of answered prompts per day. From Day 1 through 7, total response rates were $63 \%, 54 \%, 48 \%, 35 \%, 31 \%, 23 \%$, and $23 \%$ respectively $(N=$ 128 prompts per day). Over time, the total response rate decreased $40 \%$.

Figure 4: Response Rates per Time of Day $(N=30)$

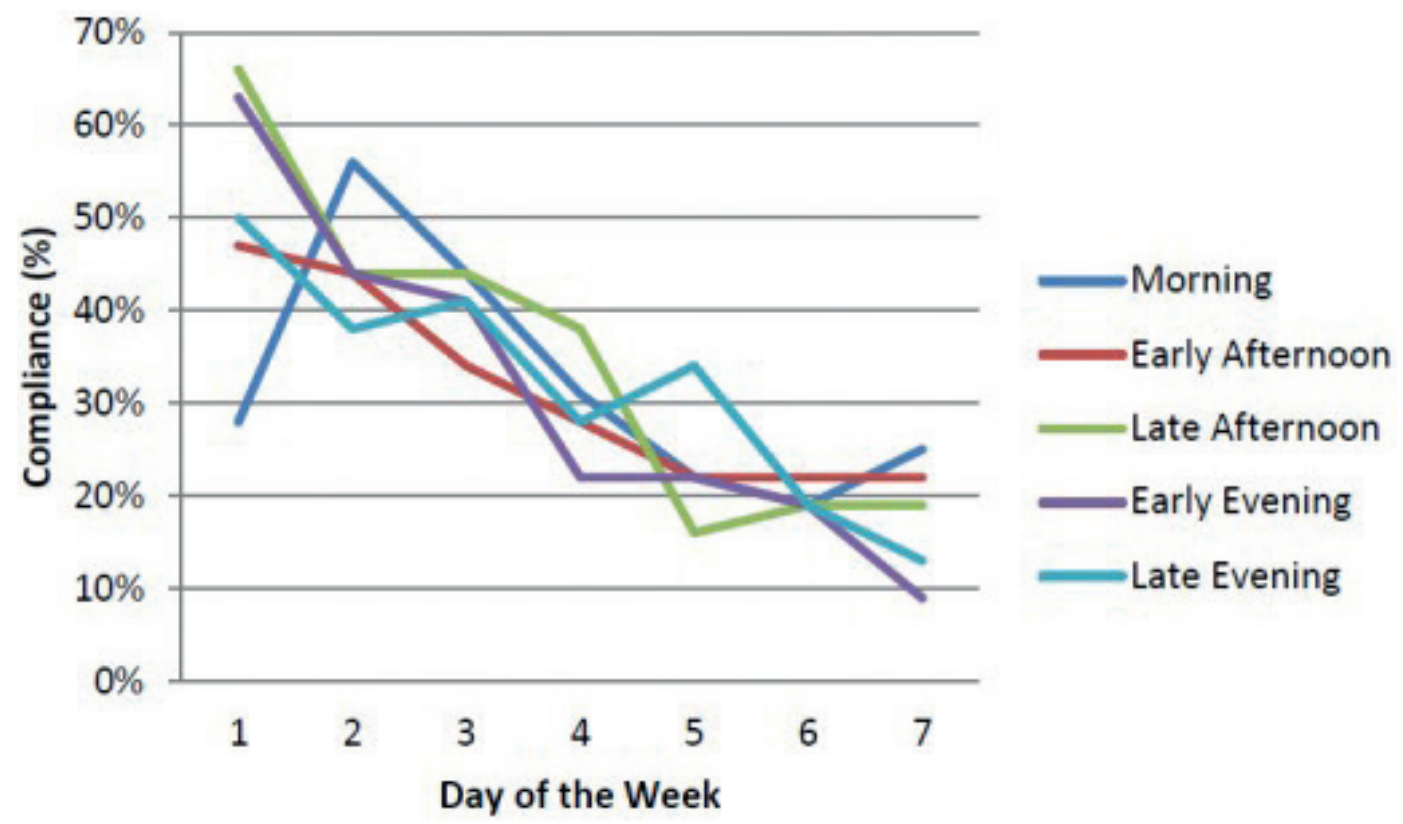

\section{Feasibility and Usability}

Of all participants $(N=30), 17$ students $(57 \%)$ completed the online evaluation form (i.e., regarding the feasibility and usability of mEMA). Normality was assessed by means of Q-Q plots. These plots showed all items to be normally distributed. The mean scores (and standard deviations) of all items are displayed in Table 1. Participants reported that the mEMA app was relatively easy to use $(M=3.88$, $S D=.93)$, that during the study they carried their smartphone with them every day $(M=4.65, S D=.61)$, and that, according to them, the mEMA app worked well $(M=3.76, S D=1.09)$. Participants who took more time to complete the mEMA app questionnaires were also more likely to report that the time required to complete them was too lengthy $(r=.154, P=.04)$. No significant differences in the perceived usability of the mEMA app between boys $(M=3.29, S D=.95)$ and girls $(M=3.90, S D=.99)$ were found $(P=.88)$. 
Table 1. Feasibility and usability measures $(N=17)$

\begin{tabular}{lcc}
\hline Item & Mean score ${ }^{\mathrm{a}}$ (SD) & Score>mean ${ }^{\mathrm{b}} \%$ \\
\hline 1. The mEMA app is easy to use. & $3.88(0.93)$ & 65 \\
2. It is easy to carry the smartphone with me. & $4.35(1.06)$ & 65 \\
3. I carried my smartphone with me every day. & $4.65(0.61)$ & 71 \\
4. After the researcher's explanation I understood how the app would & $4.24(0.75)$ & 41 \\
work. & & 59 \\
5. It was fun to work with the app. & $2.88(1.27)$ & 59 \\
6. It was boring to work with the app. & $2.88(1.27)$ & 59 \\
7. The app worked well. & $3.76(1.09)$ & 71 \\
8. I experienced the prompts as reasonable. & $3.00(1.17)$ & 53 \\
9. The number of prompts was annoying. & $3.47(1.51)$ & 71 \\
10. I filled in the mEMA app questionnaires for 7 consecutive days. & $3.76(1.35)$ & 59 \\
11. I filled in the mEMA app questionnaires 4 times a day. & $3.41(1.42)$ & 47 \\
12. It was easy to fill in the mEMA app questionnaire on my smartphone. & $4.24(0.90)$ & 71 \\
13. The questions were well-displayed on my smartphone. & $4.71(0.47)$ & 47 \\
14. Filling in a mEMA app questionnaire was an interruption of my daily & $2.59(1.46)$ & \\
activities. & & 41 \\
15. Filling in 1 mEMA app questionnaire took too long. & $2.24(1.20)$ & 35 \\
16. The study took too long. & $2.24(1.15)$ & 77 \\
17. I understood the questions that were asked. & $4.71(0.49)$ &
\end{tabular}

a Scores were based on a 5-point Likert scale (ranging from 1 - totally disagree to 5 - totally agree).

${ }^{\mathrm{b}} \mathrm{S} c o r e>$ mean illustrates the percentage of scores above the mean.

\section{Ecological Validity Analyses}

Ecological validity analyses were performed to indicate whether response categories covered all types of students' snacking in the DI condition and if response categories in the PA condition covered all types of physical activity. Table 2 shows that the consumed snacks could be categorized as cookies, pastries, fast food, sweets, chips, chocolate, nuts, cheese/sausage, popcorn, and kebab/pizza. Out of all prompts that participants responded to (128 out of 1020 prompts), 58 prompts were answered with "Yes, I have consumed an unhealthy snack during the past 3.5 hours". In total, 104 snacks were reported by participants, indicating that per snacking episode an average number of 1.8 snacks were consumed.

The reported PA could be broken down into the categories of walking, exercising, working (standing), biking, cleaning the house, doing groceries, shopping, and something else (e.g., internship at a kindergarten; see Table 2). Out of all prompts that participants responded to $(N=128), 48$ prompts 
were answered with "Yes, I have been physically active during the past 3.5 hours". In total, 97 activities were reported, indicating that during several of these 48 time periods of 3.5 hours each, approximately 2 different activities were performed.

Table 2. Secondary vocational education school students' Dietary Intake (DI) and Physical Activity (PA)

\begin{tabular}{|c|c|c|}
\hline Condition & Category & Frequency \\
\hline \multicolumn{3}{|l|}{$\mathrm{DI}(N=58)$} \\
\hline & Cookies & 19 \\
\hline & Pastry & 19 \\
\hline & Fast food & 18 \\
\hline & Sweets & 12 \\
\hline & Chips & 11 \\
\hline & Chocolate & 6 \\
\hline & Nuts & 6 \\
\hline & Cheese/Sausage & 4 \\
\hline & Ice cream & 4 \\
\hline & Popcorn & 3 \\
\hline & Kebab/Turkish Pizza & 2 \\
\hline & Total DI & 104 \\
\hline \multicolumn{3}{|l|}{$\mathrm{PA}(N=48)$} \\
\hline & Walking & 28 \\
\hline & Sporting & 22 \\
\hline & Working (standing) & 15 \\
\hline & Biking & 13 \\
\hline & Cleaning the house & 11 \\
\hline & Doing groceries & 3 \\
\hline & Shopping & 3 \\
\hline & Miscellaneous & 2 \\
\hline & Total PA & 97 \\
\hline
\end{tabular}

\section{Social and Physical Categories}

In addition to the ecological validity of response categories for DI and PA, ecological validity was analyzed for social context and location. These two context-related categories refer to the people that joined the participants and their exact location while they consumed a snack or were physically active. Results indicate that response categories for the social context fitted with $93 \%$ of all responses for DI and $98.3 \%$ for PA. Remaining responses were categorized as "Other" (including "family", "people from school", and "grandma" for DI, and "people from the gym" for PA). Regarding the loca- 
tion in which participants consumed snacks or were physically active, the developed response categories fitted with $90 \%$ for DI and 96\% for PA. Remaining responses were categorized as "Elsewhere" (including "family", "car", "theater", and "camping site" for DI, and "gym" and "in town" for PA).

Table 3 provides an overview of the categorized social contexts and locations. The overall amount of DI and PA by all participants appeared to be higher than the amount of reported social contexts and locations, indicating that participants might have eaten different snacks and have performed different types of PA in the same setting.

Table 3. Dietary Intake (DI) and Physical Activity (PA) related to social context and location

\begin{tabular}{|c|c|c|c|c|}
\hline Condition & Social Context ${ }^{\mathrm{a}}$ & $\mathbf{n}$ & Location & $\mathbf{n}$ \\
\hline \multicolumn{5}{|l|}{ DI } \\
\hline & Alone & 9 & Home & 23 \\
\hline & Friend & 9 & School & 7 \\
\hline & Colleague & 11 & Work & 12 \\
\hline & Classmate & 5 & Friend's & 4 \\
\hline & Teammate & 0 & At the streets & 5 \\
\hline & Sibling & 8 & Sports club & 1 \\
\hline & Parent & 14 & Restaurant & 2 \\
\hline & Partner & 4 & Mall & 0 \\
\hline & Child & 5 & Elsewhere & 6 \\
\hline & Teacher & 0 & - & - \\
\hline & Stranger & 0 & - & - \\
\hline \multicolumn{5}{|l|}{ PA } \\
\hline & Alone & 14 & Home & 18 \\
\hline & Friend & 10 & School & 8 \\
\hline & Colleague & 8 & Work & 11 \\
\hline & Classmate & 5 & Friend's & 8 \\
\hline & Teammate & 4 & At the streets & 8 \\
\hline & Sibling & 3 & Sports club & 14 \\
\hline & Parent & 4 & Restaurant & 0 \\
\hline & Partner & 8 & Mall & 0 \\
\hline & Child & 0 & Elsewhere & 3 \\
\hline & Teacher & 1 & - & - \\
\hline & Stranger & 2 & - & - \\
\hline
\end{tabular}

a Multiple answers are allowed per data collection point. 


\section{Discussion}

\section{Principal Findings}

In line with technological developments over the past few years, the mEMA technique is gaining in popularity with claims of better accuracy in ambulatory data collection (Brug \& Assema van, 2001). The mEMA approach offers the opportunity to assess complex health behaviors in real-time settings, in which specifically routinized behaviors are involved (e.g., DI and PA) (Stone \& Shiffman, 2002). However, the mEMA app faced several challenges that needed to be overcome in order to improve its validity. The present study showed that the mEMA app is a usable and ecologically valid tool to measure DI and PA behaviors among secondary vocational education students, but compliance is still limited.

Secondary vocational education students' self-rated compliance was $70.6 \%$ and is in accordance with compliance rates of previous electronic EMA studies (Stinson, Feldman, \& McGrath, 2006). However, the registered compliance rates of the in situ registration by the mEMA app indicated a compliance rate of only $43.8 \%$. Such a discrepancy may be explained by an availability heuristic, indicating an overestimation of compliance when students evaluate their compliance retrospectively as with the online evaluation form used in this study. According to Stone et al (2007), noncompliance might be caused by several factors, e.g. forgetting and monitoring burden (Stone et al., 2007). Up to 3 reminder signals were programmed in the mEMA app when participants did not respond, with time intervals of 30 minutes. Therefore, we assume that the chance of forgetting to respond to a mEMA prompt as a cause of noncompliance in the present study was small. Additionally, the number of prompts was reduced from 5 times a day to 4 times a day, based on feedback received from the first group. Accordingly, a significant decrease in monitoring burden was expected. However, the results from the online evaluation indicated that more than half of the students who filled in the online evaluation form (52.3\%) still experienced the number of prompts as bothersome. Therefore, monitoring burden might be a cause of the students' noncompliance in the present study. Noncompliance in the present study might also be attributable to the educational level of the students, as higher noncompliance rates have been more commonly reported among less educated students (Crutzen \& Göritz, 2010).

The usability of mEMA was rated as good. However, it should be mentioned that on the online evaluation form participants commented that the VAS-slider (see Figure $3 \mathrm{~A}, \mathrm{C}$ ) was "difficult and sometimes 
bothersome to use." These comments are in line with experiences from adolescents who participated in an electronic chronic pain diary study (Stinson et al., 2006). These adolescents also perceived the VAS slider as "hard to control" and commented that when they dragged the slider, it did not move the way they wanted. However, they also mentioned that once they discovered how to use the VAS slider more effectively, they "got the hang of it" (p. 300; Stinson et al., 2006)). Stinson et al suggested that VAS slider use could be improved by creating a thicker slider so it would move more easily, changing several sliders into radio buttons, and adding space for adolescents to enter their own responses. These adaptations were taken into account during the development of the mEMA app. But, despite these adaptations and rehearsal time prior to the study, the slider was still perceived as difficult and sometimes annoying to use. However, when the students received an explanation of the VAS sliders after they participated in the study (i.e., due to practical limitations), support for the slider increased. Therefore, irritation might be prevented by providing a good rationale prior to study, including an explanation of ways to use the slider effectively. In addition to providing a good rationale, future studies are encouraged to develop new 5-point scale designs on smartphones, in order to find a viable alternative to VAS sliders.

To our knowledge, empirical research on the ecological validity of mEMA is still lacking. In the present study, the response categories used within the contexts of DI and PA appeared to cover (almost) all responses. This indicates that the collected data represents the full range of social and physical factors of influence. These categories could be used in future mEMA research on DI and PA behavior. Besides the response categories used within the contexts of DI and PA, the MEMA app has potential use for future research on complex cognitions and (health-related) behaviors for behavioral research in general. The rationale for using mEMA rests on three central benefits of this methodology. First, retrospective recall (and the associated biases) is greatly reduced with $\mathrm{MEMA}$ because people report on current or recent states and events that occurred shortly prior to the received prompt. Second, mEMA occurs in natural settings, increasing external and ecological validity compared to clinical settings (e.g., laboratory research). Third, multiple assessments occur over time, so temporal relationships among variables can be explored (Heron \& Smyth, 2010; Shiffman et al., 2008). Fourth, the use of mobile-based EMA offers the opportunity to examine cognitions, affect, and behavior in the everyday context of people, using the natural handling and carrying of mobile phones. As such, mEMA offers a good opportunity for those behavioral, social, and health scientists and practitioners who aim to understand, intervene, and evaluate the effects of interventions on behavior (change), to examine individual differences as well as contextual differences. 


\section{Limitations}

Finally, important limitations of the present study need to be addressed. First, not all students were able to participate in the mEMA study, because not all students possessed a smartphone that operated on (recent versions of) BlackBerry, Android, or iOS. Consequently, 14 out of 44 students were exempted from further participation in the mEMA study. In order to include these exempted students in future research, a solution may be found in the (temporary) provision of eligible smartphones. Second, the mEMA app may reduce the likelihood of various biases (e.g., recall bias, availability, and recency effects), but other biases may still threaten the validity of responses, such as social desirability. Social desirability bias might play an important role in health-related behavior as, for instance, participants are expected to underreport their dietary intake and overestimate their physical activity (Brug \& Assema van, 2001; Lechner et al., 1997; Ronda et al., 2001; Sallis \& Saelens, 2000). However, Crutzen et al (2010) indicated with their study on social desirability and self-reported health risk behaviors in Web-based research that there was no meaningful association between social desirability and self-reported health risk behaviors in Web-based research (Crutzen \& Göritz, 2010). Because students in the present study used the mEMA app in real time in the absence of the researcher, it might be that a meaningful association between social desirability and self-reported DI and PA in mEMA studies is lacking too. Therefore, a comparable study on social desirability in future mEMA research might be interesting. Nevertheless, correction for these types of biases might still be necessary. Third, time-based prompting and prompting frequency were adjusted to the school's schedule. However, in the Dutch secondary vocational education system, students are required to work as interns for 2 days a week, which, according to our participants, prevented them from responding to all prompting sequences. Some students also mentioned their spring break as a reason for low compliance. Accordingly, flexible prompting (i.e., adjustable by the participant) or encouragement of user-based entries could be taken into account in future deployments.

\section{Conclusion}

Overall, mEMA offers the opportunity to assess complex health behaviors (e.g., DI and PA) in real-time settings, in which specifically routinized behaviors are involved. However, the study also revealed some challenges with regard to use of the mEMA app that need to be taken into account in order to improve its validity. In particular, compliance is a reason for concern. 


\section{Conflicts of Interest}

None declared.

\section{Abbreviations}

CMS: content management system

DI: dietary intake

EMA: Ecological Momentary Assessment

mEMA: mobile Ecological Momentary Assessment

PA: physical activity

VAS: visual analogue scale 


\section{References}

Adriaanse, M. A., Ridder de, D. T. D., \& Wit de, J. B. F. (2009). Finding the critical cue: Implementation3 intentions to change one's diet work best when tailored to personally relevant reasons for unhealthy eating. PSPB, 35(1), 60-61-71.

Bogers, R. P., Brug, J., Assema van, P., \& Dagnelie, P. C. (2004). Explaining fruit and vegetable consumption: Theory of planned behaviour and misconception of personal intake levels. Appetite, 42, 157-166.

Brug, J., \& Assema van, P. (2001). Beliefs about fat: Why do we hold beliefs about fat and why and how do we study these beliefs? In L. J. Frewer, E. Risvik \& H. Schifferstein (Eds.), Food, people and society: A european perspective of consumers' food choices. (pp. 39-54). Berlin: Springer-Verlag.

Carels, R. A., Hoffman, J., Collins, A., Raber, A. C., Cacciapaglia, H., \& O‘Brien, W. H. (2001). Ecological momentary assessment of temptation and lapse in dieting. Eating Behaviors, 2, 307-321.

Collins, R. L., Kashdan, T. B., \& Gollnisch, G. (2003). The feasibility of using cellular phones to collect ecological momentary assessment data: Application to alcohol consumption. Experimental and Clinical Psychopharmacology, 11(1), 73-78.

Cranford, J. A., Shrout, P. E., lida, M., Rafaeli, E., Yip, T., \& Bolger, N. (2006). A procedure for evaluating sensitive to within-person change: Can mood measures in diary studies detect change reliably? Personality \& Social Psychology Bulletin, 32, 917-929.

Crutzen, R., \& Göritz, A. S. (2010). Social desirability and self-reported health risk behaviors in web-based research: Three longitudinal studies. BMC Public Health, 10, 720.

Daugherty, B. L., Schap, T. E., Ettienne-Gittens, R., Zhu, F. M., Bosch, M., Delp, E. J., . . Boushey, C. J. (2012). Novel technologies for assessing dietary intake: Evaluating the usability of a mobile telephone food record among adults and adolescents. Journal of Medical Internet Research, 14(2)

De Vries, S. I., Bakker, I., van Mechelen, W., \& Hopman-Rock, M. (2007). Determinants of activity-friendly neighborhoods for children: Results from the SPACE study. Americal Journal of Health Promotion, 21(4), 312-316.

Dijkstra, A., \& Buunk, A. P. (2008). Self-evaluative emotions and expectations about self-evaluative emotions in health-behaviour change. British Journal of Social Psychology, 47, 119-137. 
Dunton, G. F., Liao, Y., Intille, S. S., Spruijt-Metz, D., \& Pentz, M. (2011). Investigating children's psychical activity and sedentary behavior using ecological momentary assessment with mobile phones. Obesity, 19, 1205-1206-1212.

Dunton, G. F., Whalen, C. K., Jamner, L. D., \& Floro, J. N. (2007). Mapping the social and physical contexts of physical activity across adolescence using ecological momentary assessment. Behavioral Medicine, 34(2), 144-153.

Grow, H. E., Saelens, B. E., Kerr, J., Durant, N. H., Norman, G. J., \& Sallis, J. F. (2008). Where are youth active? roles of proximity, active transport, and built environment. Medicine \& Science in Sports \& Exercise, , 2071-2079.

Haedt-Matt, A. A., \& Keel, P. K. (2011). Revisiting the affect regulation model of binge eating: A meta-analysis of studies using ecological momentary assessment. Psychological Bulletin, 137(4), 660-681.

Heinonen, R., Luoto, R., Lindfors, P., \& Nygard, C. (2012). Usability and feasibility of mobile phone diaries in an experimental physical exercise study. Telemedicine and E-Health, 18(2), 1-5.

Heron, K. E., \& Smyth, J. M. (2010). Ecological momentary interventions: Incorporating mobile technology into psychosocial and healthb ehaviour treatments. British Journal of Health Psychology, 1, 1-39.

Lechner, L., Brug, J., \& de Vries, H. (1997). Misconceptions of fruit and vegetable consumption: Differences between objective and subjective estimation of intake. Journal of Nutrition Education, 29(6), 313-320.

Mays, D., Cremeens, J., Usdan, S., Martin, R. J., Arriola, K. J., \& Bernhardt, J. M. (2010). The feasibility of assessing alcohol use among college students using wireless mobile devices: Implications for ehalth education and behavioural research. Health Education Journal, 69(3), 311-320.

Nordgren, L. F., van der Pligt, J., \& van Harreveld, F. (2008). The instability of health cognitions: Visceral states influence self-efficacy and related health beliefs. Health Psychology, 27(6), 722-727.

Ozdalga, E., Ozdalga, A., \& Ahuja, N. (2012). The smartphone in medicine: A review of current and potential use among physicians and students. Journal of Medical Internet Research, 14(5), e128. 
Prins, R. G., Empelen van, P., Beenackers, M. A., Brug, J., \& Oenema, A. (2010). Systematic development of the YouRAction program, a computer-tailored physical activity promotion intervention for dutch adolescents, targeting personal motivations and environmental opportunities. BMC Public Health, 10,474 .

Rofey, D. L., Hull, E. E., Phillips, J., Vogt, K., Silk, J. S., \& Dahl, R. E. (2010). Utilizing ecological momentary assessment in pediatric obesity to quantify behavior, emotion, and sleep. Behavior and Psychology, 18(6), 1270-1272.

Ronda, G., van Assema, P., \& Brug, J. (2001). Stages of change, psychological factors and awareness of physical activity levels in the netherlands. Health Promotion International, 16(4), 305-314.

Sallis, J. F., \& Saelens, B. E. (2000). Assessment of physical activity by self-report: Status, limitations, and future directions. Research Quarterly for Exercise and Sport, 71(2), S1-14.

Schnall, R., Okoniewski, A., Tiase, V., Low, A., Rodriguez, M., \& Kaplan, S. (2013). Using text messaging to assess adolescents' health information needs: An ecological momentary assessment. Journal of Medical Internet Research, 15(3), e54.

Serre, F., Fatseas, M., Debrabant, R., Alexandre, J., Auriacombe, M., \& Swendsen, J. (2012). Ecological momentary assessment in alcohol, tobacco, cannabis and opiate dependence: A comparison of feasibility and validity. Drug and Alcohol Dependence, 126, 118-123.

Shiffman, S., Stone, A. A., \& Hufford, M. R. (2008). Ecological momentary assessment. Annual Review of Clinical Psychology, 4, 1-32.

Stinson, J. N., Feldman, B. M., \& McGrath, P. J. (2006). E-ouch:Usability testing of an electronic pain diary for adolescents with arthritis. Clinical Journal of Pain, 22, 295-305.

Stone, A. A., \& Shiffman, S. (1994). Ecological momentary assessment in behavioral medicine. Annals of Behavioral Medicine, 16, 199-202.

Stone, A. A., Shiffman, S., Atienza, A. A., \& Nebeling, L. (2007). The science of real-time data capture. self-reports in health research. New York: Oxford University Press.

Stone, A.A., \& Shiffman, S. (2002). Capturing momentary self-report data: A proposal for reporting guidelines. Annals of Behavioral Medicine, 24, 236-243. 
Thomas, J. G. (2009). Toward a better understanding of the development of overweight: A study of eating behavior in the natural environment using ecological momentary assessment.

Thomas, J. G., Doshi, S., Crosby, R. D., \& Lowe, M. R. (2011). Ecological momentary assessment of obesogenic eating behavior: Combining person-specific and environmental predictors. Obesity, 19, 1574-1579.

Tsai, C., Lee, G., Raab, F., Norman, G. J., Sohn, T., Griswold, W. G., \& Patrick, K. (2007). Usability and feasibility of PmEB: A mobile phone application for monitoring real time caloric balance. Mobile Network Application, 12, 173-184.

White, M. A., Whisenhunt, B. L., Williamson, D. A., Greenway, F. L., \& Netemeyer, R. G. (2002). Development and validation of the food-craving inventory. Obesity Research, 10(2), 107-114.

Zhang, S., Wu, Q., van Velthoven, M. H. M. M. T., Chen, L., Car, J., Rudan, I., . . Scherpbier, R. W. (2012). Smartphone versus pen-and-paper data collection of infant feeding practice in rural china. Journal of Medical Internet Research, 14(5), e119. 



\section{CHAPTER 3}

The Role of Motivational and Contextual Factors on Intentional and

Craving-Induced Snack Consumption: A mobile-based Ecological

Momentary Assessment (mEMA) Study

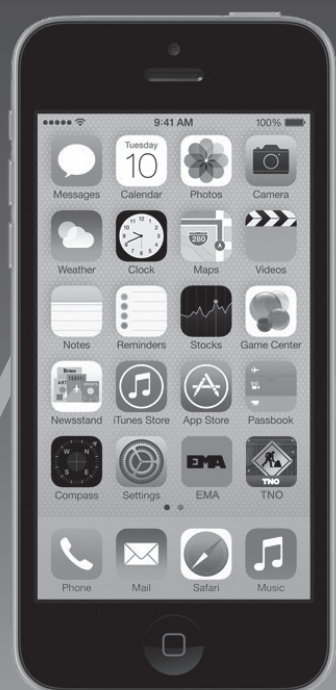




\title{
The Role of Motivational and Contextual Factors on Intentional and Craving-Induced Snack Consumption: A mobile-ased Ecological Momentary Assessment (mEMA) Study
}

\begin{abstract}
Objective: In the present mobile-based Ecological Momentary Assessment (mEMA) study, motivational and contextual factors were examined as predictors of low socio-economic status (SES) youth' snack consumption (SC) from a dual process model perspective (i.e., intention and self-reported craving).

Design: Eighty participants (68\% female) were prompted by the mEMA app four times a day during seven consequtive days, asking them to review their SC and psychological, physical, and social factors of SC with a timeslot of 3.5hours.

Results: Multilevel analyses (i.e., time of day nested within individuals) showed a significant increase in SC during the day, and a positive association between self-reported craving and SC (OR = 16.1, $95 \% \mathrm{Cl}$ 9.11-28.35). Additional analyses indicated that hunger ( $\mathrm{OR}=2.0,95 \% \mathrm{Cl} 1.37-2.91)$ and snack intention ( $\mathrm{OR}=1.5,95 \% \mathrm{Cl} 1.00-2.20)$ were associated with craving-induced SC. Snack intention was associated with subsequent SC (OR $=2.5,95 \% \mathrm{Cl} 1.65-3.68)$.

Conclusion: Snack consumption increases during the day, and is associated with immediate food craving. Snack intention was not associated with immediate SC, but was related to subsequent SC.
\end{abstract}

Keywords: snack consumption; craving-induced snack consumption; snack intention; mobile-based Ecological Momentary Assessment (mEMA); cross-time effects

\section{Introduction}

Recent studies have indicated that overweight and obesity are major global health concerns (Finucane et al., 2011; Wang, McPherson, Marsh, Gortmaker, \& Brown, 2011). Overweight and obesity are associated with several negative health outcomes (i.e., diabetes, cardiovascular disease and some forms of cancer (Formiguera \& Canton, 2004; Roberts, Deleger, Strawbridge, \& Kaplan, 2003; World Health Organization [WHO], 2004) and increased health care costs (Polder \& Takkern, 2002). A frequently associated behavior with overweight is the intake of high energy-dense food, or snack consumption (SC; Phillips et al., 2004; Slater et al., 2009; Swinburn et al., 1998). As a reduction of this SC may prevent individuals from (excessive) weight gain, studying (determinants of) snack consumption (SC) is important (Hendriksen, Boer, Du, Feskens, \& van der A, 2011; Swinburn, Caterson, Seidell, 
\& James, 2004). Especially SC among youth from low Socio Economic Status (SES) is alarming, as SC is common and cumulating among this group (Wang, Monteiro, \& Popkin, 2002; Wang et al., 2007). Corresponding findings within the Netherlands showed that especially among youth from non-western cultural backgrounds overweight and obesity rates are concerning (Schönbeck \& Buuren van, 2010). As $16 \%$ of youth with a Dutch background is overweight or obese, rates from youth with a Moroccan or Turkish background are respectively $34 \%$ and $40 \%$.

Previous studies on eating behavior have been performed from a social cognitive perspective, which considers that intentions are the most proximal determinant of health behavior (Ajzen, 1991). Nevertheless, intentions do often not result in related behaviors (Webb \& Sheeran, 2006). A recent systematic review by McEachan, Conner, Taylor, and Lawton (2011) suggest that the Theory of Planned Behavior (TPB), which has been the dominant theoretical approach to guide research on health-related behavior for the past few decades, accounted for $19.3 \%$ of variability in health behavior, and was considerably less predictive in longitudinal studies (McEachan, Conner, Taylor, \& Lawton, 2011). These health behaviors are mainly determined by intentions for as far as individuals have control over their behavior (Fishbein \& Ajzen, 2010), but the intention - health behavior relationship is weaker among individuals from lower SES groups (Conner et al., 2013). In relation to SC, the remaining rather uncontrolled desire to eat and the expected rewarding, pleasurable consequence of consuming the food item is generally defined as food craving (Hill, Weaver, \& Blundell, 1991; Nijs, Franken, \& Muris, 2007; Rogers, Anderson, Finch, Jas, \& Gatenby, 1994; Rozin, Levine, \& Stoess, 1991; Weingarten \& Elston, 1991). Food craving may jeopardize self-regulation of eating behavior and is positively related to body weight (Franken \& Muris, 2005; Herman \& Polivy, 2004). Both craving-induced SC and intentions are likely to be influenced by motivational and contextual factors (Hofmann, Friese, \& Wiers, 2008). As such, the aim of the present study is to understand which factors might influence SC among low SES youth.

Research up to date has indicated a number of relevant factors that may contribute to SC. Patel and Schlundt (2001) have, first of all, indicated that people generally eat more when they are in a positive or negative mood compared to when they are in a neutral mood (Patel \& Schlundt, 2001). However, Patel and Schlundt also indicated that the impact of the social context is large (effect size around 50\%) compared to the impact of moods (effect size around 9\%; Patel \& Schlundt, 2001). The social facilitation of food intake literature has consistently illustrated that food intake increases 
when meals are eaten in a the presence of others (Clendenen, Herman, \& Polivy, 1994; De Castro, 1991; De Castro, 1994; Patel \& Schlundt, 2001; Spook, Paulussen, Kok, \& van Empelen, 2013). Next to the social setting, students frequently reported that they consumed energy-dense snacks at home, at school, or at work (i.e., physical setting) (Adriaanse, de Ridder, \& de Wit, 2009). In these settings it has been indicated that the availability of snacks increased snack consumption (Pearson et al., 2012), as well as through vending machines and fast food restaurants within walking distance from schools (Neumark-Sztainer, French, Hannan, Story, \& Fulkerson, 2005; Zenk \& Powell, 2008).

However, most of these findings relied on retrospective self-reports. Self-report measures of complex health behaviors are generally subject to inaccuracy, systematically biased recall, and social desirability effects (Stone, Shiffman, \& de Vries, 2000). Also poor or faked compliance and incomplete data are returning methodological issues (Broderick, Schwartz, Shiffman, Hufford, \& Stone, 2003; Ronda, van Assema, \& Brug, 2001; Stone, Shiffman, Atienza, \& Nebeling, 2007). A methodology that encounters these biases is mobile-based Ecological Momentary Assessment (mEMA).

mEMA enables cross-time assessment of complex health behaviors and related factors of influence in real-time and real-world settings (Stone et al., 2007). mEMA is especially useful for behaviors that fluctuate within an individual, over time and across situations (Stone et al., 2007). In the present mEMA study we aimed to gain a better understanding of to what extent snack consumption 1) fluctuates over time, 2) can be explained by snack intention and craving, 3) which motivational and contextual factors contribute to explaining SC directly, or indirectly via intention or craving, and 4) how snack intention and craving are associated with SC over time (Figure 1). It should be noted that we measured "self-reported craving", as previously operationalized by e.g. Grenard et al. (Grenard et al., 2013), and Pelchat and Schaefer (Pelchat \& Schaefer, 2000), and that craving and hunger are suggested to be two different phenomenons, in which hunger is not a necessary condition for food craving (Pelchat, Johnson, Chan, Valdez, \& Ragland, 2004). 
Figure 1: Theoretical Framework

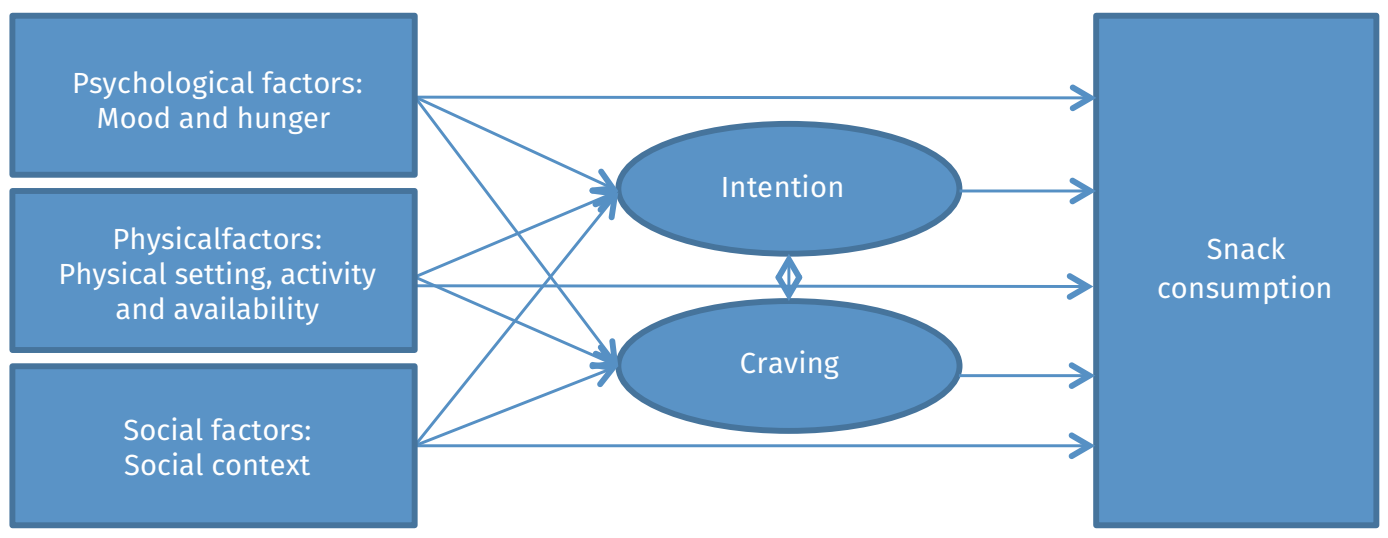

\section{Method}

\section{Participants}

Eighty students from seven secondary vocational education schools in the Netherlands participated in the current mEMA study. The sample was $59 \%$ female $^{1}$ and $41 \%$ minority race. The mean age of participants was $19.4(S D=2.3$, range 16-25), and Body Mass Index (BMI) ranged from 17.7 to 28.1 $(M=22.5, S D=2.9)$.

\section{Procedure}

All students who participated in the present study started with an online baseline survey regarding the average amount and types of food they consumed during a week. After completion, they received an email with a description on download and log-in procedures of the mEMA app. Those students who possessed a smartphone that operated at BlackBerry OS, Android or iOS (i.e., the most commonly used platforms among secondary vocational education students in September 2011 (Spook et al., 2013) downloaded the mEMA app on their smartphone and followed the instructions about log-in procedures. Students who possessed a smartphone with other operating systems or did not own a smartphone were exempt from further participation. Participants received a short training on how to use the app and they started using the app the next day, for seven consecutive days. During these days, participants were requested to fill out short questionnaires regarding their dietary intake during the preceding 3.5 hours, four times a day. Participatory incentives of ten $€ 20$ coupons were randomly distributed among the students. All students consented to take part in the study 
and students aged $\leq 17$ were provided with a passive consent form for their parents to complete. All procedures were approved by The Ethical Committee of Psychology (Maastricht University).

\section{Measures}

mEMA permits the assessment of complex health behaviors in real-time and ecological valid settings (Stone et al., 2007), indicating that assessments take place in participants' natural environments, are related to their current state (e.g., current mood, current behavior), and involve repeated assessments over time (Haedt-Matt \& Keel, 2011). Previous research already showed that a mEMA application (app) is a feasible, usable and ecologically valid tool to monitor SC, indicating that a mEMA app can be used to monitor this complex health behavior (Spook et al., 2013). Therefore, mEMA has been used as research method in the present study.

The mEMA questionnaire included assessment of: (1) Snack consumption - a 12-item scale preceded by a stem: Which and how many snacks did you have during the previous 3.5 hours? Response options were nuts, chips, popcorn, cheese/sausage, pastry, cookies, sweets, chocolate, fastfood, kebab/Turkish pizza, icecream, and other. Additionally, each response included the possibility to enter the exact number of snacks they had consumed; cf. (Adriaanse et al., 2009; Spook et al., 2013) (2) Intention - a 1-item scale operationalized as: Are you planning to eat a snack during the upcoming 3.5 hours?. Response options ranged from ' 1 ', definitely not to ' 5 ', definitely; cf. (Carels et al., 2001) (3) Self-Reported craving - a 1-item scale operationalized as: Did you feel the need for a snack during the previous 3.5 hours?. Response options were dichotomous, "no"/"yes" (0/1); cf. (White, Whisenhunt, Williamson, Greenway, \& Netemeyer, 2002) (4) Mood - a 10-item scale preceded by a stem: At the moment, I'm feeling.. Items were operationalized as happy, tired, stressed, bored, satisfied, sad, frustrated, angry, lonely, and lively. Response options to each item contained a 5-point semantic differential scale, ranging from ' 1 ', not at all to ' 5 ', very much. All scales were merged into 1 mood scale, and response options were dichotomized as "mainly negative mood"/"mainly positive mood" for the analyses; cf. (Carels et al., 2001) (5) Hunger - a 1-item scale operationalized as: How hungry were you during the previous 3.5 hours?. Response options to each item contained a 5-point semantic differential scale, ranging from ' 1 ', not at all to ' 5 ', very much. Response options were dichotomized as "not hungry"/"hungry" for the analyses; cf. (Carels et al., 2001) (6) Physical setting - a 1-item scale operationalized as: Where were you when having a snack?. Response options included at home, school, work, a friends' place, outdoors, a restaurant, cafeteria, supermarket, sports club, and else- 
where. Response options were dichotomized as "at home"/"elsewhere" for the analyses; cf. (Spook et al., 2013) (7) Physical activity - a 1-item scale operationalized as: What were you doing when having a snack?. Response options included watching TV, relaxing, working, phone, playing games on the PC, doing homework, got a class, doing groceries, travel, clubbing, shopping, chatting, texting, nothing, other. Response options were dichotomized as "inactive"/"active" for the analyses; cf. (Adriaanse et al., 2009) (8) Availability - a 1-item scale operationalized as: How many snacks were available during the previous 3.5 hours?. Response options to each item contained a 5-point semantic differential scale, ranging from ' 1 ', not at all to ' 5 ', very much. Response options were dichotomized as “low availability"/"high availability" for the analyses; cf. (Thomas, 2009) (9) Presence of others (social context) a 1-item scale operationalized as: How many peers were with you while having a snack? Response options were alone, with one other person, and with multiple other persons Response options were dichotomized as "alone"/"with others" for the analyses; cf. (Spook et al., 2013).

\section{Data Analyses}

All statistical multilevel analyses were carried out using SPSS 20 General Linear Mixed Models (GLMM). Data was nested in timeslots (i.e., time of day) within participants. After model comparison, descriptive statistics, and univariate analyses, multivariate analyses were conducted including significant correlates of SC. Also cross-time associations are analyzed by means of GLMM.

\section{Results}

\section{Compliance with the Study Protocol}

Out of 2240 initial prompts ( $N=80,4$ prompts a day during 7 consecutive days) sent to participants, $32 \%$ of these prompts were answered. On average, the students participated for 3 days $(M=3.12, S D$ 1.98). Compliance rates per day decreased significantly over a 1 -week period from $99 \%$ to $25 \%$, indicating that at day one, $99 \%$ of all participants filled in at least one mEMA questionnaire, and one out of four students filled out at least one questionnaire at day seven.

\section{Descriptive Statistics}

Of all 663 reported time points, 31\% was answered with "yes, I have consumed a snack during the previous 3.5 hours". Most frequently reported snacks were small cookies (14\%), chips (12\%), chocolate $(12 \%)$, sweets $(11 \%)$, and fastfood (11\%). Participants also reported that snacks in general were 
consumed more often when they were hungry (33\%), away from home (37\%), had not been physically active during the same time interval (34\%), snacks were highly available (44\%), when they were alone (40\%), when they had strong intentions to consume a snack $(34 \%)$, and especially when they experience food craving (70\%). Participants did not report more snack consumption when they were positive or negative mooded (both $33 \%$ ).

\section{Within-Person Fluctuations}

Results indicated a significant main effect for time of day $(F(1,659)=15.21, p=.000)$. Snack consumption (SC) increased during the day, respectively during the early afternoon ( $\mathrm{OR}=1.9,95 \% \mathrm{Cl} 1.07-3.34$ ), late afternoon ( $\mathrm{OR}=4.1,95 \% \mathrm{Cl} 2.32-7.29)$, and evening $(\mathrm{OR}=5.0,95 \% \mathrm{Cl}$ 2.96-8.30). Therefore, we controlled for time effects in all further analyses. We also checked for several background variables (i.e., BMI, age, gender, and ethnicity), but none of these effects was significant.

\section{Univariate Associations Between Intrapersonal and Contextual Factors Associated with SC}

First, we assessed univariate effects of all predictors on SC controlled for time effects (Table 1). Results indicated (borderline) significant main effects for self-reported craving $(F(1,658)=162.74, p$ $=.000)$, physical activity $(F(1,567)=6.58, p \leq .05)$, the presence of others $(F(1,658)=3.96, p \leq .05)$ and availability of snacks $(F(1,411)=3.82, p=.05)$. Snack consumption among students was more likely when they experienced food craving, $(\mathrm{OR}=16.3,95 \% \mathrm{Cl} 10.59-24.98)$, when snacks were available $(\mathrm{OR}=$ 1.7, $\mathrm{Cl} 1.00-2.74)$, or when they had been less physically active ( $\mathrm{OR}=0.5,95 \% \mathrm{Cl} .31-.86)$, but less likely when they were in the presence of other people ( $\mathrm{OR}=0.6,95 \% \mathrm{Cl} .40-.99)$. No significant main effects were found for mood, hunger, and intention. 
Table 1: Univariate analyses of all predictors on snack consumption, intention, and self-reported craving $(N=80)$

\begin{tabular}{|c|c|c|c|c|c|c|c|}
\hline Outcome variable & Predictor variable & $\mathbf{F}$ & df & $\mathbf{p}$ & B & $\mathbf{O R}^{*}$ & $95 \% \mathrm{Cl}$ \\
\hline \multirow[t]{5}{*}{ Snack consumption } & Time of day & 15.21 & 3,659 & .000 & & & \\
\hline & (Morning; REF) & & & & 1.00 & & \\
\hline & (Early afternoon) & & & & .64 & 1.9 & $1.07-3.34$ \\
\hline & (Late afternoon) & & & & 1.41 & 4.1 & $2.32-7.29$ \\
\hline & (Evening) & & & & 1.60 & 5.0 & $2.96-8.30$ \\
\hline \multirow[t]{5}{*}{ Intention } & Time of day & 7.30 & 3,611 & .000 & & & \\
\hline & (Morning; REF) & & & & 1.00 & & \\
\hline & (Early afternoon) & & & & .00 & 1.0 & $.61-1.62$ \\
\hline & (Late afternoon) & & & & .14 & 1.2 & $.68-1.92$ \\
\hline & (Evening) & & & & $(-.81)$ & 0.4 & $.28-.70$ \\
\hline \multirow[t]{5}{*}{ Craving } & Time of day & 11.79 & 3,707 & .000 & & & \\
\hline & (Morning; REF) & & & & 1.00 & & \\
\hline & (Early afternoon) & & & & .57 & 1.8 & $1.04-3.03$ \\
\hline & (Late afternoon) & & & & 1.35 & 3.9 & $2.27-6.58$ \\
\hline & (Evening) & & & & 1.22 & 3.4 & $2.11-5.49$ \\
\hline \multirow[t]{21}{*}{ Snack Consumption } & Physical setting & .79 & 1,633 & .38 & & & \\
\hline & (Home; REF) & & & & 1.00 & & \\
\hline & (Elsewhere) & & & & $(-.17)$ & 0.8 & $.58-1.23$ \\
\hline & Physical activity & 6.58 & 1,567 & .01 & & & \\
\hline & (Inactive; REF) & & & & 1.00 & & \\
\hline & (Active) & & & & $(-.67)$ & 0.5 & $.31-.86$ \\
\hline & Presence of others & 3.96 & 1,658 & .05 & & & \\
\hline & (Alone; REF) & & & & 1.00 & & \\
\hline & (With others) & & & & $(-.46)$ & 0.6 & $.40-.99$ \\
\hline & Availability & 3.82 & 1, 411 & .05 & & & \\
\hline & (Not available; REF) & & & & 1.00 & & \\
\hline & (Available) & & & & .50 & 1.8 & $1.00-2.74$ \\
\hline & Mood & .97 & 1,628 & .32 & & & \\
\hline & (Negative affect; REF) & & & & 1.00 & & \\
\hline & (Positive affect) & & & & $(-.27)$ & 0.8 & $.45-1.31$ \\
\hline & Hunger & .02 & 1,613 & .89 & & & \\
\hline & (Not hungry; REF) & & & & 1.00 & & \\
\hline & (Hungry) & & & & .03 & 1.0 & $.70-1.52$ \\
\hline & Intention & .28 & 1,609 & .60 & & & \\
\hline & (Low intention; REF) & & & & 1.00 & & \\
\hline & (High intention) & & & & $(-.11)$ & 0.9 & $.60-1.35$ \\
\hline
\end{tabular}




\begin{tabular}{|c|c|c|c|c|c|c|c|}
\hline Outcome variable & Predictor variable & $\mathbf{F}$ & df & $\mathbf{p}$ & B & $\mathbf{O R}^{*}$ & $95 \% \mathrm{Cl}$ \\
\hline & Self-Reported craving & 162.74 & 1,658 & .000 & & & \\
\hline & (No craving; REF) & & & & 1.00 & & \\
\hline & (Craving) & & & & 2.79 & 16.3 & $10.59-24.98$ \\
\hline \multirow[t]{21}{*}{ Intention } & Physical setting & .00 & 1,607 & .95 & & & \\
\hline & (Home; REF) & & & & 1.00 & & \\
\hline & (Elsewhere) & & & & $(-.01)$ & 1.0 & $.68-1.43$ \\
\hline & Phyiscal activity & .30 & 1,546 & .58 & & & \\
\hline & (Inactive; REF) & & & & 1.00 & & \\
\hline & (Active) & & & & .13 & 1.1 & $.72-1.79$ \\
\hline & Presence of others & 5.06 & 1,610 & .025 & & & \\
\hline & (Alone; REF) & & & & 1.00 & & \\
\hline & (With others) & & & & $(-.54)$ & 0.6 & $.36-.93$ \\
\hline & Availability & 10.08 & 1,406 & .002 & & & \\
\hline & (Not available; REF) & & & & 1.00 & & \\
\hline & (Available) & & & & .81 & 2.3 & $1.36-3.74$ \\
\hline & Mood & 6.50 & 1,584 & .01 & & & \\
\hline & (Negative affect; REF) & & & & 1.00 & & \\
\hline & (Positive affect) & & & & .68 & 2.0 & $1.17-3.32$ \\
\hline & Hunger & 48.14 & 1,602 & .000 & & & \\
\hline & (Not hungry; REF) & & & & 1.00 & & \\
\hline & (Hungry) & & & & 1.36 & 3.9 & $2.65-6.72$ \\
\hline & Self-Reported craving & 3.14 & 1,610 & .08 & & & \\
\hline & (No craving; REF) & & & & 1.00 & & \\
\hline & (Craving) & & & & .35 & 1.4 & $.96-2.11$ \\
\hline \multirow[t]{13}{*}{ Craving } & Physical setting & .87 & 1,636 & .35 & & & \\
\hline & (Home; REF) & & & & 1.00 & & \\
\hline & (Elsewhere) & & & & $(-.17)$ & 0.8 & $.59-1.21$ \\
\hline & Phyiscal activity & .29 & 1,571 & .59 & & & \\
\hline & (Inactive; REF) & & & & 1.00 & & \\
\hline & (Active) & & & & .13 & 1.1 & $.72-1.79$ \\
\hline & Presence of others & .96 & 1,706 & .33 & & & \\
\hline & (Alone; REF) & & & & 1.00 & & \\
\hline & (With others) & & & & .20 & 1.2 & $.82-1.84$ \\
\hline & Availability & .23 & 1,412 & .63 & & & \\
\hline & (Not available; REF) & & & & 1.00 & & \\
\hline & (Available) & & & & .11 & 1.1 & $.70-1.78$ \\
\hline & Mood & .04 & 1,561 & .85 & & & \\
\hline
\end{tabular}




\begin{tabular}{|c|c|c|c|c|c|c|c|}
\hline Outcome variable & Predictor variable & $\mathbf{F}$ & df & $\mathbf{p}$ & B & $\mathbf{O R}^{*}$ & $95 \% \mathrm{Cl}$ \\
\hline & (Negative affect; REF) & & & & 1.00 & & \\
\hline & (Positive affect) & & & & .05 & 1.1 & $.64-1.74$ \\
\hline & Hunger & 12.87 & 1,614 & .000 & & & \\
\hline & (Not hungry; REF) & & & & 1.00 & & \\
\hline & (Hungry) & & & & .69 & 2.0 & $1.37-2.91$ \\
\hline & Intention & 3.94 & 1,610 & .05 & & & \\
\hline & (Low intention; REF) & & & & 1.00 & & \\
\hline & (High intention) & & & & .40 & 1.5 & $1.00-2.20$ \\
\hline
\end{tabular}

* Controlled for time of day; bold Odd Ratios are significant $(p \leq .05)$

\section{Multivariate Associations Between Intrapersonal and Contextual Factors With SC}

Next, a multivariate multilevel regression model was examined (see Table 2). Physical activity, the presence of others, availability of snacks, and self-reported craving were included in the multivariate model as they were found to be (borderline) significantly related to snack consumption. Using a stepwise approach, we first entered all (borderline) significant background variables in the multivariate model (i.e., physical activity, presence of others, and availability of snacks). Results only indicated a borderline significant main effect for the presence of others, indicating that snack consumption among students was less likely to occur when they were in the presence of others $(\mathrm{OR}=0.5,95 \% \mathrm{CI}$ .29-1.00). When self-reported craving was added to the model, results indicated that participants were approximately 15 times more likely to consume snacks when they experienced food craving (OR $=14.8,95 \% \mathrm{Cl} 8.56-25.72)$. Physical activity, the presence of others and availability of snacks were not significantly associated with snack consumption ( $n s)$. 
Table 2: Stepwise multivariate analyses of physical activity, presence of others, and availability of snacks on snack consumption $(N=80)$

\begin{tabular}{|c|c|c|c|c|c|c|c|c|}
\hline Step & Outcome variable & Predictor variable & $\boldsymbol{F}$ & df & $p$ & B & OR & $95 \% \mathrm{Cl}$ \\
\hline \multirow[t]{9}{*}{1} & Snack consumption & Physical activity & .78 & 1,353 & .38 & & & \\
\hline & & (Inactive; REF) & & & & 1.00 & & \\
\hline & & (Active) & & & & $(-.27)$ & 0.8 & $.41-1.40$ \\
\hline & & Presence of others & 3.93 & 1,353 & .05 & & & \\
\hline & & (Alone; REF) & & & & 1.00 & & \\
\hline & & (With others) & & & & $(-.63)$ & 0.5 & $.29-1.00$ \\
\hline & & Availability & 3.93 & 1,353 & .25 & & & \\
\hline & & (Not available; REF) & & & & 1.00 & & \\
\hline & & (Available) & & & & .32 & 1.4 & $.80-2.36$ \\
\hline \multirow[t]{12}{*}{2} & Snack consumption & Physical activity & 2.94 & 1,352 & .09 & & & \\
\hline & & (Inactive; REF) & & & & 1.00 & & \\
\hline & & (Active) & & & & $(-.63)$ & 0.5 & $.26-1.10$ \\
\hline & & Presence of others & 3.09 & 1,352 & .08 & & & \\
\hline & & (Alone; REF) & & & & 1.00 & & \\
\hline & & (With others) & & & & $(-.64)$ & 0.5 & $.26-1.08$ \\
\hline & & Availability & 1.35 & 1,352 & .25 & & & \\
\hline & & (Not available; REF) & & & & 1.00 & & \\
\hline & & (Available) & & & & .37 & 1.5 & .78-2.69 \\
\hline & & Craving & 92.98 & 1,352 & .000 & & & \\
\hline & & (No craving; REF) & & & & 1.00 & & \\
\hline & & (Craving) & & & & 2.70 & 14.8 & $8.56-25.72$ \\
\hline
\end{tabular}

\section{Predictors of Intention and Self-Reported Craving}

In additional analyses the background variables and intention were regressed on self-reported craving. Results only showed a significant association between hunger and self-reported craving (OR $=2.0,95 \% \mathrm{Cl}$ 1.37-2.91), and a borderline significant association between snack intention and self-reported craving $(\mathrm{OR}=1.5,95 \% \mathrm{Cl} 1.00-2.20)$. Snack intention was less likely to occur when students were in the presence of others $(\mathrm{OR}=0.6,95 \% \mathrm{Cl} .36-.93)$, but more likely when snacks were available $(\mathrm{OR}=2.3,95 \% \mathrm{Cl} 1.36-3.74)$, students were in a positive mood $(\mathrm{OR}=2.0,95 \% \mathrm{Cl} 1.17-3.32)$, or were hungry $(\mathrm{OR}=3.9,95 \% \mathrm{Cl} 2.65-6.72)$. 


\section{Order Effects of Intrapersonal Factors for Snack Consumption}

Finally, we explored order effects of SC associated with intrapersonal factors (i.e., cognitive factors, affective factors, and the perceived environmental factors) across time by means of multilevel time lagged analyses. By calculating this mean, we sought to understand the direction of the relationships between intention, self-reported craving, and SC. Table 3 shows associations between (1) factors and the outcome variable at a specific time slots $\left(T_{0}\right),(2)$ factors at a specific time slots and the outcome criterion at the first following time slots $\left(T_{+1}\right)$, and (3) outcome criterion at a specific time slot and factors at the first following time slot $\left(T_{-1}\right)$. Results indicate that snack intention $\left(T_{0}\right)$ was positively associated with subsequent $\mathrm{SC}\left(\mathrm{T}_{1}\right)(\mathrm{OR}=2.5,95 \% \mathrm{Cl} 1.65-3.68)$, and self-reported craving was associated with SC during the same timeslot $\left(\mathrm{T}_{0}\right)(\mathrm{OR}=16.3,95 \% \mathrm{Cl} 2.36-3.22)$. Other cross-time associations were not significant, indicating that snack consumption was not associated with subsequent snack intentions or self-reported craving.

Table 3: Order Effects of Intrapersonal Factors for Snack Consumption

\begin{tabular}{|c|c|c|c|c|c|c|}
\hline \multirow[b]{2}{*}{ Predictor Variable } & \multicolumn{6}{|c|}{ Snack Consumption } \\
\hline & $\mathbf{O R}_{\mathrm{T}+1}$ & $95 \% \mathrm{Cl}_{\mathrm{T}+1}$ & $\mathrm{OR}_{\mathrm{TO}}$ & $95 \% \mathrm{Cl}_{\mathrm{TO}}$ & $O R_{T-1}$ & $95 \% \mathrm{Cl}_{\mathrm{T}-1}$ \\
\hline Intention & 2.5 & $1.65-3.68$ & 0.9 & $-.52-.30$ & 1.0 & $.65-1.40$ \\
\hline Craving & 1.2 & $.82-1.72$ & 16.3 & $2.36-3.22$ & 0.8 & $.54-1.15$ \\
\hline
\end{tabular}

* bold is significant $(p \leq .05)$

\section{Discussion}

We examined if snack consumption could be explained by intentions and craving. In addition, we assessed the influence of psychological, physical and social factors on snack consumption (SC), self-reported craving and intentions, and cross-time associations between intention, selfreported craving, and SC. The present study showed that SC increased significantly during the day, and that SC was most likely to occur when students experienced food craving, and less likely when they were in the presence of other people. We also showed that snack intention predicted subsequent SC, whilst self-reported craving was associated with SC during the same timeslot. Of notice, snack intention was positively related to food craving, as well as with various motivational and contextual factors (i.e., the availability of snacks, presence of others, negative affect, and feeling hungry). 
It is worth noticing that snack consumption increased during the day. A possible explanation can be derived from the strength model of self-control by Baumeister and colleagues. This model suggest that people have a limited energy source to actively engage in self-control. Once people are depleted, they loose the capacity to regulate their behavior. Our finding may suggest that people become depleted over time, and are therefore more likely to have a snack later on (e.g., in the evening) (Baumeister, Vohs, \& Tice, 2007). As our finding shows that snack consumption is more likely to be explained by self-reported craving than intentions, people might be more likely to go with their impulses when they are depleted.

Moreover, the fluctuation in self-reported snack consumption during the day is of interest as it underscores the need for more precise measurements of behaviors, other than the more traditional self-report questionnaires. Clearly, self-reports may be highly dependent on the time of day and, therefore, self-reports in traditional questionnaires may include an over- or underestimation of the behavior as they reflect on, for example, snack consumption over the last seven days. Traditional self-report questionnaires are also commonly constructed from a social cognitive perspective, indicating that health behavior is mainly predicted by intention (Webb \& Sheeran, 2006). However, no clear evidence was found for the direct association of intention and snack consumption within the present mEMA study. Therefore, future research including a more hybrid approach (i.e., survey and mEMA) might be desirable, as it would allow for a comparison between survey and real-time data.

Despite the methodological benefits of using mEMA to measure determinants of secondary vocational education students' snack consumption, the present study has some limitations. First, results might not be generalizable to the total student population, as the target population specifically involves low SES students (i.e., secondary vocational education students). Previous research by Conner et al. (2013) indicated that the intention-health behavior relationship can be attenuated in lower SES samples, because they are less engaged with health behaviors and poorer at translating their healthy intentions into behavior (Conner et al., 2013). Second, craving was operationalized by means of self-reported craving, possibly resulting in more reflective craving as we explicitely asked students about their craving. Although, self-reported craving is a common operationalization in dietary research (Grenard et al., 2013; Pelchat \& Schaefer, 2000), it is still likely that craving remains partly unexplained because it occurred impulsively. Third, due to a right-skewed distribution of the dependent variable (i.e. snack consumption), and the assumption that consuming (unhealthy) snacks 
increases the chance of weight gain in general, the dependent variable was dichotomized for all analyses. Consequently, no differentiations can be made between small and large snack episodes in certain situations. Therefore, future research on a subsample of snack consumption (e.g., small portions versus large portions) might contribute to our overall understanding of associations between individual and contextual factors and snack consumption proportions.

A final limitation was students' lack of compliance. As mentioned in our previous feasibility study, monitoring burden might be a cause of students' noncompliance, but might also be attributable to their educational level as noncompliance appears more frequent among lower educated students (Crutzen \& Göritz, 2010). During the development of the mEMA app, seven behavioral science principles were used to enhance subject compliance to EMA protocols: build compliance into the protocol, subject training, user interface issues, programmed reminders, guide subjects through assessments, incorporate data collection systems into their daily lives ("livability" functions), and create a sense of accountability (Stone et al., 2007). We anticipated accurately to the first six principles, but as the mEMA app was initially developed as a research tool, no feedback system was incorporated within the app. According to the seventh principle, compliance rates increase when participants feel accountable for the data, when they are aware of the fact that data collection is being tracked, and they receive real-time feedback regarding their compliance. Because the mEMA app lacked any feedback loops, compliance in future mEMA research might increase if feedbackmechanisms are included.

Nevertheless, the present study contributes to the theoretical debate concerning the explanation of health behavior and provides support for a real-time ecological approach. Snack consumption is mainly determined by the impulsive system which, in turn, is stronger when students intend to have a snack. When students are in the presence of others they have lower snack intentions and are less likely to consume a snack. Therefore, desirable components for future overweight prevention and intervention programs should focus on the development and/or improvement of selfregulatory skills in order to withstand food temptations and to prevent impulsive snack consumption.

\section{Conclusion}

The present mEMA study showed that snack consumption increases during the day and is mainly predicted by self-reported craving. This reflection of the impulsive system, however, is partly 
explained by the controlled system (i.e., intention), which in turn seems to be predicted by contextual factors such as the availability of snacks and the presence of others. Therefore, we conclude that the presence of others can play an important role in the prevention of snack consumption and that the controlled and impulsive system within dual process models do not operate fully independent, but snack intention rather operates as a food craving facilitator.

\section{Endnotes}

${ }^{1} 13 \%$ of participants $(N=80)$ did not report their age. 


\section{References}

Adriaanse, M.A., de Ridder, D.T.D., \& de Wit, J.B.F. (2009). Finding the Critical Cue: Implementation Intentions to Change One's Diet Work Best when Tailored to Personally Relevant Reasons for Unhealthy Eating. PSPB, 35(1), 60-71.

Ajzen, I. (1991). The theory of planned behavior. Organizational Behavior and Human Decision Processes, 50, 179-211.

Baumeister, R.F., Vohs, K.D., \& Tice, D.M. (2007). The strength model of self-control. Psychological Science, 16, 351-355.

Broderick, J.E., Schwartz, J.E., Shiffman, S., Hufford, M.R., \& Stone, A.A. (2003). Signaling does not adequately improve diary compliance. Annals of Behavioral Medicine, 26,139-148.

Carels, R.A., Hoffman, J., Collins, A., Raber, A.C., Cacciapaglia, H., \& O’Brien, W.H. (2001). Ecological momentary assessment of temptation and lapse in dieting. Eating Behaviors, 2, 307-321.

Clendenen, V. I., Herman, P. C., \& Polivy, J. (1994). Social facilitation of eating among friends and strangers. Appetite, 23, 1-13.

Conner, M., McEachan, R., Jackson, C., McMillan, B., Woolridge, \& M., Lawton, R. (2013). Moderating effect of socioeconomic status on the relationship between health cognitions and behaviors. Annals Behavioral Medicine, 46, 19-30.

Crutzen, R., \& Göritz, A.S. (2010). Social desirability and self-reported health risk behaviors in web-based research: Three longitudinal studies. BMC Public Health, 10, 72.

De Castro, J. M. (1991). Social facilitation of the spontaneous meal size of humans occurs on both weekdays and weekends. Physiology \& Behavior, 49, 1289-1291.

De Castro, J. M. (1994). Family and friends produce greater social facilitation of food intake than other companions. Physiology \& Behavior, 56(445), 455.

Finucane, M.M., Stevens, G.A., Cowan, M.J., Danaei, G., Lin, J.K., Paciorek, C.J., Singh, G.M., Gutierrez, H.R., Lu, Y., Bahalim, A.M., Farzadfar, F., Riley, L.M., Ezzati, M., \& Global Burden of Metabolic Risk Factors of Chronic Diseases Collaborating Group (Body Mass Index) (2011). National, regional, and global trends 
in body-mass index since 1980: systematic analysis of health examination suveys and epidemiological studies with 960 country-years and 9.1 million participants. Lancet, 377, 557-567.

Fishbein, M., \& Ajzen, I. (2010). Predicting and changing behavior: The reasoned action approach. New York: Psychology Press (Taylor \& Francis).

Formiguera, X., \& Canton, A. (2004). Obesity: Epidemiology and clinical aspects. Best Practice and Research Clinical Gastroenterol, 18(6), 1125-1146.

Franken, I.H.A., \& Muris, P. (2005). Individual differences in reward sensitivityare are related to food craving and relative body weight in healthy women. Appetite, 45, 198-201.

Grenard, J.L., Stacy, A.W., Shiffman, S., Baraldi, A.N., MacKinnon, D.P., Lockhart, G., Kisbu-Sakarya, Y., Boyle, S., Beleva, Y., Koprowski, C., Ames, S.L., \& Reynolds, K.D. (2013). Sweetened drink and snack cues in adolescents. A study using ecological momentary assessment. Appetite, 67, 61-73.

Haedt-Matt, A.A., \& Keel, P.K. (2011). Revisiting the affect regulation model of binge eating: a meta-analysis of studies using ecological momentary assessment. Psychological Bulletin, 137(4), 660-681.

Hendriksen, M.A.H., Boer, J.M.A., Du, H., Feskens, E.J.M., \& van der A, D.L. (2011). No consistent association between consumption of energy-dense snack foods and annual weight and waist circumference changes in Dutch adults. American Journal of Clinical Nutrition, 94, 19-25.

Herman, C.P., \& Polivy, J. (2004). The self-regulation of eating: Theoretical and practical problems. In: Baumeister, R.F., \& Vohs, K.D., editors. Handbook of self-regulation: Research, theory, and applications. New York: Guilford.

Hill, A.J., Weaver, C.F., \& Blundell, J.E. (1991). Food craving, dietary restraint and mood. Appetite, 17:187197.

Hofmann, W., Friese, M., \& Wiers, R.W. (2008). Impulsive versus reflective influences on health behavior: a theoretical framework and empirical review. Health Psychology Review, 2(2), 111-137.

McEachan, R.R.C., Conner, M., Taylor, N., \& Lawton, R.J. (2011). Prospective prediction of health-related behaviors with the Theory of Planned Behavior: A meta-analysis. Health Psychology Review, 5, 97-144. 
Neumark-Sztainer, D., French, S.A., Hannan, P.J., Story, M., Fulkerson, J.A. (2005). School lunch and snacking pattern among high school students: Associations with school food environment and policies. International Journal of Behavioral Nutrition and Physical Activity, 2, 14.

Nijs, I.M.T., Franken, I.H.A., \& Muris, P. (2007). The modified Trait and State Food-Cravings Questionnaire: Development and validation of a general index of food craving. Appetite, 49, 38-46.

Patel, K. A., \& Schlundt, D. G. (2001). Impact of moods and social context on eating behavior. Appetite, $36,111-118$.

Pearson, N., Biddle, S.J.H., Williams, L., Worsley, A., Crawford, D., \& Ball, K. (2012). Adolescent television viewing and unhealthy snack food consumption: the mediating role of home availability of unhealthy snack foods. Public Health Nutrition, 17(2), 317-323.

Pelchat, M.L., \& Schaefer, S. (2000). Dietary monotony and food cravings in young and elderly adults. Physiology \& Behavior, 68, 353-359.

Phillips, S. M., Bandini, L. G., Naumova, E. N., Cyr, H., Colclough, S., Dietz, W. H., \& Must, A. (2004). Energy-dense snack food intake in adolescence: Longitudinal relationship to weight and fatness. Obesity Research, 12(3), 461-472.

Polder, J.J., \& Takkern, J. (2002). Kosten van Ziekten in Nederland - De zorgeuro ontrafeld [Cost of illness in the Netherlands], p. 304.

Roberts, R.E., Deleger, S., Strawbridge, W.J., \& Kaplan, G.A. (2003). Prospective association between obesity and depression: evidence from the Alameda County Study. International Journal of Obesity Related Metabolism Disorders, 27(4), 514-521.

Rogers, P., Anderson, A., Finch, G.M., Jas, P., \& Gatenby, S.J. (1994). Relationships between food craving and anticipatory salivation, eating patterns, mood and body weight in women and men. Appetite, 23, 319.

Ronda, G., van Assema, P., \& Brug, J. (2001). Stages of change, psychological factors and awareness of physical activity levels in the Netherlands. Health Promotion International, 16(4), 305-314.

Rozin, P., Levine, E., \& Stoess, C. (1991). Chocolate craving and liking. Appetite, 17, 199-212. 
Schönbeck, Y., \& van Buuren, S. (2010). Factsheet resultaten vijfde landelijke groeistudie. Leiden, TNO.

Slater, J., Green, C. G., Sevenhuysen, G., Edginton, B., O’Neil, J., \& Heasman, M. (2009). The growing canadian energy gap: More the can than the couch? Public Health Nutrition, 12(11), 2216-2224.

Spook, J.E., Paulussen, T., Kok, G., \& van Empelen, P. (2013). Monitoring dietary intake and physical activity electronically: feasibility, usability, and sensitivity of a mobile-based Ecological Momentary Assessment (mEMA) tool. Journal of Medical Internet Research, 15(9), e214.

Stone, A.A., Shiffman, S., Atienza, A.A., \& Nebeling L. (2007). The science of real-time data capture. Self-reports in health research. New York: Oxford University Press.

Stone, A. A., Shiffman, S., \& de Vries, M. W. (2000). Ecological momentary assessment. In D. Kahneman, E. Diener \& N. Schwarz (Eds.), Well-being: The foundations of hedonic psychology. (pp. 26-39). New York: Russell Sage Foundation.

Swinburn, B.A., Caterson, I., Seidell, J.C., \& James, W.P. (2004). Diet, nutrition and the prevention of excess weight gain and obesity. Public Health Nutrition, 7, 123-146.

Swinburn, B.A., Sacks, G., Lo, S.K., Westerterp, K.R., Rush, E.C., Rosenbaum, M., Luke, A., Schoeller, D.A., DeLany, J.P., Butte, N.F., Ravussin, E. (1998). Estimating the changes in energy flux that characterize the rise in obesity prevalence. American Journal of Clinical Nutrition, 89(6), 1723-1728.

Thomas, J.G. (2009). Toward a better understanding of the development of overweight: a study of eating behavior in the natural environment using ecological momentary assessment (PhD thesis). Drexel University, Philadelphia.

Wang, Y., Liang, H., Tussing, L., Braunschweig, C., Cabellero, B., \& Flay, B. (2007). Obesity and related risk factors among low socio-economic status minority students in Chicago. Public Health Nutrition, 10(9), 927-938.

Wang, Y., Monteiro, C., \& Popkin, B.M. (2002). Trends of obesity and underweight in older children and adolescents in the United States, Brazil, China, and Russia. The American Journal of Clinical Nutrition, 75, 971-977.

Wang, Y.C., McPherson. K., Marsh, T., Gortmaker, S.L., Brown, M. (2011). Health and economic burden of the projected obesity trends in the USA and the UK. Lancet, 378, 815-825. 
Webb, T.L., \& Sheeran, P. (2006). Does changing behavioral intentions engender behavior change? A meta-analysis of the experimental evidence. Psychological Bulletin, 132(2), 249-268.

Weingarten, H.P., \& Elston, D. (1991). Food cravings in a college population. Appetite, 17, 167-175.

White, M.A., Whisenhunt, B.L., Williamson, D.A., Greenway, F.L., Netemeyer, R.G. (2002). Development and validation of the Food-Craving Inventory. Obesity Research, 10(2), 107-114.

World Health Organization [WHO] (2003). Diet, nutrition and the prevention of chronic diseases. Geneva.

Zenk, S.N., \& Powell, L.M. (2008). US secondary schools and food outlets. Health \& Place, 14(2), 336-346. 



\section{CHAPTER 4}

Factors Determining Physical Activity of Emerging Young Adults:

A mobile-based Ecological Momentary Assessment (mEMA) Study

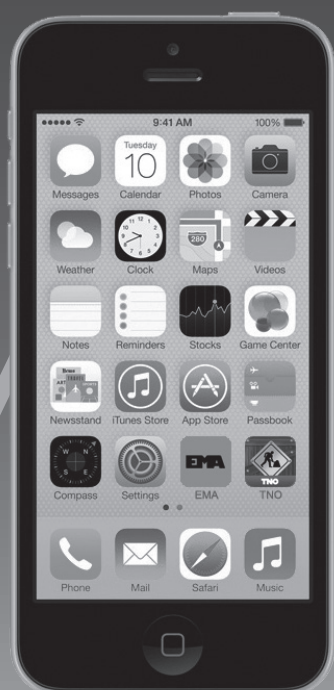




\section{Factors Determining Physical Activity of Emerging Young Adults: A mobile-based Ecological Momentary Assessment (mEMA) Study}

\section{Abstract}

Background: Many emerging young adults struggle to carry out a sufficient amount of physical activity (PA). Therefore, the aim of the present mobile-based Ecological Momentary Assessment (mEMA) study was to gain a better understanding of the underlying motivational (cognitive and affective) and contextual (physical and social environmental) determinants of walking, cycling, and exercising, and to find out when these effects occur.

Methods: Over the course of seven consecutive days, 71 participants (64\% female) received prompts from the mEMA-app four times a day, containing questions regarding cognitive (i.e., intention, perceived behavioral control (PBC), perceived environment), affective, and contextual factors (i.e., physical and social environment) that may have influenced their PA during the previous 3.5 hours. Multilevel analyses (i.e., time of day nested within individuals) and exploratory multilevel timelagged analyses were conducted.

Results: PA behaviors were associated with mood (e.g., walking $\left(\mathrm{OR}_{\text {feelinglively }}=1.7,95 \% \mathrm{Cl} 1.05-2.87\right)$, the physical setting (e.g., cycling $\left(\mathrm{OR}_{\text {schoolsetting }}=1.8,95 \% \mathrm{Cl} 1.02-3.18\right)$, and the social environment (e.g., exercise $(\mathrm{OR}=1.8,95 \% \mathrm{Cl}$ 1.06-3.19). Time-lagged analyses revealed that although intention and $\mathrm{PBC}$ were not directly associated with PA, they did predict subsequent PA (e.g., exercise $\left(\mathrm{OR}_{\text {intention } \mathrm{T}+1}=2.7\right.$, 95\% Cl 1.56-4.60).

Conclusions: Although contextual factors are related to concurrent PA behaviors, motivational factors (intention, PBC and affect) determine subsequent PA behaviors. As such, it is important to take into account that cognitive factors and behaviors fluctuate over time, which may explain differences in relationships between cognitions and PA behaviors as shown in previous cross-sectional studies.

Keywords: Physical Activity, mobile-based Ecological Momentary Assessment (mEMA), Cross-Time Effects, Socioecological Model 


\section{Introduction}

Regular physical activity (PA, i.e. walking, cycling and exercising) is associated with multiple health benefits, such as reduced risk of becoming overweight or obese, musculoskeletal health improvement (i.e., higher bone density), improvement of psychological well-being, and healthier cardiovascular risk profiles (Boreham \& Riddoch, 2001; The European Heart Initiative, 2001). Additionally, it has been shown that physically active youth are more likely to become physically active adults (Telama et al., 2005). However, PA declines strikingly with age (Physical activity and fitness, 2010), especially during adolescence and young adulthood (Caspersen, Pereira, \& Curran, 2000). Approximately 65\% of high school students (14-18 years old) engage in regular vigorous activity, as compared to $32 \%$ of 18-24-year-olds and $23 \%$ of adults (Physical activity and fitness, 2010). In a similar way, approximately $27 \%$ of high school students engage in moderate activity; again, this figure drops to $17 \%$ of 18-24-year-olds, and to 15\% of adults (Physical activity and fitness, 2010; Rovniak, Anderson, Winett, \& Stephans, 2002). This decline in PA occurs during a transition stage from adolescence to adulthood, which has also been referred to as emerging adulthood (Arnett, 2000). The aim of the present study was to understand which factors might promote PA among this group of emerging adults.

A considerable number of studies have used social-cognitive theories to explain PA behavior (e.g., (Ajzen, 1991). These motivational models generally assume that behavior is guided by intentions and perceived behavioral control (PBC). In other words, these models assume that conscious decision making and the perceived ability to perform a behavior guide PA. Although these models have been useful in explaining health behavior, only $12 \%$ of objectively measured PA among youth can be explained by PA intentions (Hagger, Chatzisarantis, \& Biddle, 2002; Motl et al., 2002; Prins, 2012; Sallis \& Owen, 2002). As such, most behaviors in everyday life (e.g., PA) are most likely influenced by other factors, both motivational (e.g., affect and the perceived environment) and contextual (i.e., the physical and social environment) (Ajzen, 2011; Davidson \& Lawson, 2006; Ekkekakis \& Petruzzello, 2000; Prins, Oenema, van der Horst, \& Brug, 2009; Prins, 2012; Sallis, Johnson, Calfas, Caparosa, \& Nichols, 1997; Spence \& Lee, 2003). Although a significant number of studies have explored these associations between motivational factors and PA, and contextual factors and PA, it should be noted that these studies have targeted different populations (e.g., adolescents; (Motl, Birbaum, Kubik, \& Dishman, 2004), and have mainly relied on retrospective self-reports (e.g., Penedo \& Dahn, 2005; Prins et al., 2009; Romero, 2005). 
Retrospective reporting on complex behaviors such as PA is relatively difficult, as it is subject to inaccuracy, systematically biased recall, and social desirability effects (Stone, Shiffman, \& de Vries, 2000). Recall problems are more likely for behaviors that are frequent and irregular, intensive, and that change over time, and for those that have to be reflected upon within the specific context in which the behavior was performed (Stone, Shiffman, Atienza, \& Nebeling, 2007). Furthermore, studies that rely on retrospective self-reported data are generally subject to poor or faked compliance, and incomplete data (e.g., missing data / ambiguous responses) (Broderick, Schwartz, Shiffman, Hufford, \& Stone, 2003; Ronda, van Assema, \& Brug, 2001; Stone et al., 2007). With regard to affective states, there may also be a discrepancy between affect at the time of measurement and at the time of behavioral performance (Ajzen, 2011). One methodological tool that addresses some of these limitations is mobile-based Ecological Momentary Assessment (mEMA).

\section{The Present mEMA Study}

The present study uses mobile-based Ecological Momentary Assessments (mEMA) to study the effect of motivational (i.e., cognitive and affective) and contextual (i.e., physical and social environmental) factors on emerging young adults' PA in real-time and in real-world settings. This extends previous cross-sectional research suggesting that motivational factors (e.g., affect) and PA are interrelated (Motl et al., 2004; Penedo \& Dahn, 2005) Importantly, by using mEMA, we attempted to minimize the methodological limitations of retrospective self-reported data and to provide more accurate measures of a complex behavior (i.e., PA) that fluctuates within an individual and also across situations and individuals (Dunton, Whalen, Jamner, Henker, \& Floro, 2005; Spook, Paulussen, Kok, \& van Empelen, submitted for publication; Stone et al., 2007). As the Dutch guidelines for PA are different for moderate PA (e.g., walking and cycling) and vigorous PA (e.g. exercising; see (Bernaards \& van Buuren, 2012), we studied the determinants of emerging young adults' walking, cycling, and exercising separately (instead of studying emerging young adults' total PA).

\section{Materials and Methods}

\section{Participants}

A total of 71 students participated in the present mEMA study. The majority of the students (64\%) were female, mean age 21 years old $(S D=3.3)$. Of all participants, $96 \%$ reported that they were Dutch. $71 \%$ had a normal Body Mass Index (BMI; $M=22.9 ; S D=3.77)$. 


\section{Procedure}

Students were recruited online and at schools, and gave their consent for participation prior to the study. In cases where students were under 18 years old, passive consent forms for their parents or caregivers were provided. Students started with an online baseline survey about PA-behaviors and PA-related determinants. Upon completion of the survey, students received an email with a description of download and log-in procedures of the mEMA app. If their smartphone operated on Android, iOS, or Blackberry, they were able to download the app and to log in. Next, participants received a short training session on how to use the app and what was expected from them during the following seven days. In order to encourage participation, ten incentives of $€ 20$ were randomly distributed among participants (chance of winning 1:7). The procedure was approved by The Research Ethics Board of the School of Psychology \& Neuroscience (Maastricht University).

\section{The mEMA App}

The mEMA app prompted students four times a day over seven consecutive days. All prompts were formatted as auditory signals and were displayed on participants' smartphone screens. Time-based prompts were set around four anchor time points (i.e., 08:00 a.m., 12:00 noon, 05:00 p.m., and 09:00 p.m.) with one hour intervals, meaning that with regard to the 08:00 a.m. anchor, for example, participants could receive prompts between 07:00 a.m. and 09:00 a.m. Students were asked to reflect on their PA behaviors and associated factors during the previous 3.5 hours. When an entry was missed or rejected, up to two reminders were sent, the first after 30 minutes and the second after 60 minutes. In addition to these time-based prompts, it was possible for students to use the mEMA app whenever they wanted to (event-based entries).

\section{Measures}

The mEMA questionnaire included items regarding PA (type and frequency), intention and PBC, affect, the perceived environment, the physical environment, and the social environment. All questions were administered in Dutch.

Physical Activity (PA). PA items contained multiple choice response categories including walking, biking, and exercising. Each response category was supplemented with an additional field for recording how long (the number of minutes) the participant performed the particular behavior. 
Items were derived from previous research investigating PA using ecological momentary assessment (Dunton, Whalen, Jamner, \& Floro, 2007; Dunton, Liao, Intille, Spruijt-Metz, \& Pentz, 2011).

Motivational Factors. Perceived Behavioral Control was operationalized as: “Do you feel capable of engaging in physical activity within the next three and half hours?" A 5-point scale with response categories ranging from 1 ("certainly not") to 5 ("yes, certainly") was used (following (Prins et al., 2013). Intention was operationalized as: "Do you intend to star engaging in / remain engaged in physical activity within the next three and half hours?" Again, a 5-point scale with response categories ranging from 1 ("certainly not") to 5 ("yes, certainly") was used (Prins et al., 2013).

The Perceived Environment. Perceived availability of PA facilities was operationalized as: "Which facilities were available for you to engage in physical activity in the last three and half hours?" Multiple choice answers included "a forest", "a park", "paths", "fitness equipment", and "other facilities, namely.." (following (Dunton et al., 2007; Popkin, Duffey, \& Gordon-Larsen, 2005). Perceived safety was operationalized as: "Did you feel safe in the neighborhood that you were in during the last three and half hours?", and contained a dichotomous response category ("no/yes") (Andersen, Crespo, Bartlett, Cheskin, \& Pratt, 1998; Prins et al., 2013).

Affective Factors. Affect was assessed using a 10-item scale preceded by a question stem: At the moment, I'm feeling... Items were related to happiness, fatigue, stress, boredom, satisfaction, sadness, frustration, anger, loneliness, and liveliness. These items were adapted from a mood scale that has been used in prior mEMA research (Carels et al., 2001). Responses to all items were measured using 5-point semantic differential scales, ranging from 1 ("not at all") to 5, ("very much"), but were dichotomized for all analyses due to skewed distributions. Response categories were visualized by sliders (see Spook et al. 2013).

The Physical Environment. Physical environment was operationalized as: "Where were you while performing the behavior?" Response options included the following settings: home, school, work, a friend's place, outdoors, restaurant, cafeteria, supermarket, sports club, and elsewhere. Rainy weather was operationalized as: "Did it rain in the last three and half hours?" and contained a dichotomous response category ("no/yes"). An additional option was to enter "I don't know". This item was 
derived from coping plans as applied by the YouRAction intervention protocol (Prins, van Empelen, Beenackers, Brug, \& Oenema, 2010).

The Social Environment. The social environment was operationalized as: "How many peers were present while performing the behavior?" Response categories included: "alone", "with one other person", and "with multiple others". Responses were dichotomized into: "alone" and "with others" for the analyses (cf. Spook et al., 2013).

Data Analyses. SPSS 20.0 was used to carry out all statistical multilevel analyses using General Linear Mixed Models (GLMM). The study had a between person design in which data was nested within timeslots (i.e. time of day) within participants. Statistical models were compared prior to the analyses. Descriptive statistics as well as correlations between all factors are reported, as well as crosstime associations.

\section{Results}

\section{Compliance With the Study Protocol}

Initially, 71 participants were prompted four times a day over the course of seven consecutive days. In total, they responded to 868 out of the 1988 prompts that were sent (44\% compliance). On average, the students participated for 3.36 days $(S D=2.03)$. Over the seven days, compliance rates decreased from $71 \%$ on day one to $27 \%$ on day seven. Over the course of the day, the amount of responses varied from $43 \%$ in the morning, to $39 \%$ in the early afternoon, $32 \%$ in the late afternoon, and $61 \%$ in the evening.

\section{Descriptive Statistics}

Overall, of the 868 entries made by the total sample $(N=71), 506$ prompts were answered with "no $P A "$. The other entries encompassed 169 walking entries ( $M=35.35, S D=31.18, M d n=25$ minutes), 149 cycling entries $(M=24.40, S D=16.18, M d n=20$ minutes $)$, and 82 exercise entries $(M=63.32, S D=37.26$, $M d n=60$ minutes). It should be noted that there is an overlap between these PA frequencies as 47 participants reported multiple activities in a single time slot. Next, all outcome variables and all independent variables are dichotomized due to a violation of the normality assumption.

Students reported that they were most likely to walk during the late afternoon $(\mathrm{OR}=3.0,95 \% \mathrm{CI}$ 
1.90-6.67), to cycle during the late afternoon ( $\mathrm{OR}=2.4,95 \% \mathrm{Cl} 1.39-4.29)$, and to exercise during the late afternoon and evening $\left(\mathrm{OR}_{\text {late afternoon }}=3.5,95 \% \mathrm{Cl} 1.69-7.20 ; \mathrm{OR}_{\text {evening }}=3.6,95 \% \mathrm{Cl} 1.90-6.67\right)$. These results did not differ between subgroups (e.g., gender, age, educational level, BMI (healthy versus unhealthy weight)). All contextual factors reported are displayed in Table 1.

Table 1: Frequencies of Reported Environmental Factors

\begin{tabular}{llllllllll}
\hline & \multicolumn{1}{l}{ Factor } & \multicolumn{2}{c}{ No PA } & \multicolumn{2}{c}{ Walking } & \multicolumn{3}{c}{ Cycling } & \multicolumn{2}{c}{ Exercising } \\
\hline \multirow{2}{*}{$\begin{array}{l}\text { The Physical } \\
\text { Environment }\end{array}$} & The home environment & 373 & $(63 \%)$ & 51 & $(21 \%)$ & 42 & $(21 \%)$ & 24 & $(22 \%)$ \\
\cline { 2 - 9 } & The school environment & 61 & $(10 \%)$ & 25 & $(10 \%)$ & 26 & $(13 \%)$ & 6 & $(5 \%)$ \\
\cline { 2 - 9 } & The work environment & 77 & $(13 \%)$ & 33 & $(14 \%)$ & 21 & $(10 \%)$ & 13 & $(12 \%)$ \\
\cline { 2 - 9 } & Elsewhere & 85 & $(14 \%)$ & 134 & $(55 \%)$ & 114 & $(56 \%)$ & 67 & $(61 \%)$ \\
\cline { 2 - 9 } & Total (Physical settings) & 596 & $(100 \%)$ & 243 & $(100 \%)$ & 203 & $(100 \%)$ & 110 & $(100 \%)$ \\
\cline { 2 - 9 } $\begin{array}{l}\text { The Social } \\
\text { Environment }\end{array}$ & Rain & 99 & $(100 \%)$ & 43 & $(100 \%)$ & 36 & $(100 \%)$ & 15 & $(100 \%)$ \\
\hline
\end{tabular}

\section{Univariate Associations Between Intrapersonal and Contextual Factors Associated With PA}

Walking. Of the 22 factors included, 6 were significantly associated with walking. The results showed that walking was associated with feelings of happiness ( $O R=1.5,95 \% \mathrm{Cl} 1.05-2.19)$ and liveliness ( $O R=1.7,95 \% \mathrm{Cl} 1.14-2.42)$, the work environment ( $\mathrm{OR}=1.7,95 \% \mathrm{Cl} 1.06-2.77)$, and the presence of others (OR $=1.6,95 \% \mathrm{Cl} 1.09-2.34)$. In turn, students were less likely to walk in the perceived availability of parks ( $\mathrm{OR}=0.6,95 \% \mathrm{Cl} .37-.99)$ and in the home environment (OR $=0.1,95 \% \mathrm{Cl} .10-.22)$. Walking was not associated with intention and PBC, the perceived availability of forests, perceived safety, other affect factors (i.e., fatigue, stress, boredom, satisfaction, sadness, frustration, anger, and loneliness), or with the contextual factors school environment, and rain.

Cycling. Of the 21 factors included, 4 determinants were significantly associated with cycling. Results showed that cycling was associated with feelings of happiness ( $O R=1.5,95 \% \mathrm{Cl} 1.01-2.27$ ) and loneliness $(\mathrm{OR}=1.8,95 \% \mathrm{Cl} 1.15-2.75)$, and the school environment $(\mathrm{OR}=1.9,95 \% \mathrm{Cl} 1.06-3.29)$. Students were less likely to cycle in the perceived availability of parks (OR $=0.44,95 \% \mathrm{Cl} .25-.80)$. The home environment was not included in the analyses, as it is evident that cycling does not take place in the home setting. No other factors were associated with cycling (i.e., intention, PBC, the perceived availability of forests, perceived safety, fatigue, stress, boredom, satisfaction, sadness, frustration, anger, liveliness, the work environment, rain, or the social environment). 
Exercising. Of the 22 factors included, 5 determinants were significantly associated with exercising. Results showed that exercise was associated with feelings of happiness ( $\mathrm{OR}=1.9,95 \% \mathrm{Cl} 1.15-3.01$ ), satisfaction ( $\mathrm{OR}=2.7,95 \% \mathrm{Cl} 1.65-4.53)$, and liveliness ( $\mathrm{OR}=1.9,95 \% \mathrm{Cl} 1.15-3.01)$. Moreover, exercise was associated with being in the presence of others ( $O R=3.6,95 \% \mathrm{Cl} 2.10-6.03)$. Students were less likely to exercise in the home environment ( $\mathrm{OR}=0.1,95 \% \mathrm{Cl} .08-.21)$. Exercising was not associated with intention, PBC, the perceived availability of forests or parks, perceived safety, fatigue, stress, boredom, sadness, frustration, or anger, the work environment or rain.

\section{Multivariate Associations Between Intrapersonal and Contextual Factors With PA}

Multivariate models were separately conducted for each outcome variable (i.e., walking, cycling and exercising), based on the significant findings of the univariate models. The results of these full multivariate models are visualized in Table 2).

Walking. The multivariate model for walking included the six variables "perceived availability of parks", "feeling happy", "feeling lively", "the home environment", "the work environment", and "the social environment". Of these, only feeling lively was positively associated with walking (OR = 1.7, 95\% $\mathrm{Cl}$ 1.05-2.87), and a significant negative relationship was found between the home environment and walking (OR $=0.1,95 \% \mathrm{Cl} .06-.16)$.

Cycling. The multivariate model for cycling included the factors: "perceived availability of parks" "feeling happy", "feeling lonely", and "the school environment". Results showed that feeling happy $(\mathrm{OR}=1.6,95 \% \mathrm{Cl} 1.05-2.38)$, feeling lonely $(\mathrm{OR}=1.9,95 \% \mathrm{Cl} 1.21-3.18)$, and the school setting $(\mathrm{OR}=1.8$, $95 \% \mathrm{Cl} 1.02-3.18$ ) were positively associated with cycling, and that cycling was negatively associated with the perceived availability of parks (OR $=0.5,95 \% \mathrm{Cl} .28-.85)$.

Exercising. The multivariate model for walking included the five variables: "feeling happy", "feeling satisfied", "feeling lively", "the home environment", and "the social environment". Of these, feeling satisfied was positively associated with exercising ( $\mathrm{OR}=2.7,95 \% \mathrm{Cl} 1.47-4.94)$, as was the social context $(\mathrm{OR}=1.8,95 \% \mathrm{Cl} 1.06-3.19)$. On the other hand, however, a negative association with exercising was found for the home environment $(\mathrm{OR}=0.1,95 \% \mathrm{Cl} .06-.18)$. 
Table 2: Multivariate Associations Between Significant Intrapersonal and Contextual Factors and $P A(N=72)^{1}$

\begin{tabular}{llcc}
\hline Outcome Measure & Determinant & OR & $\mathbf{9 5 \% ~ C l}$ \\
\hline Walking & Perceived availability of parks & 0.9 & $.50-1.61$ \\
& Feeling happy & 0.9 & $.59-1.52$ \\
& Feeling lively & $\mathbf{1 . 7}$ & $1.05-2.87$ \\
& In the home environment & $\mathbf{0 . 1}$ & $.06-.16$ \\
& In the work environment & 0.7 & $.38-1.23$ \\
& In the presence of others & 1.0 & $.64-1.58$ \\
& & & \\
\hline Cycling & Perceived availability of parks & $\mathbf{0 . 5}$ & $.27-.85$ \\
& Feeling happy & $\mathbf{1 . 6}$ & $1.05-2.38$ \\
& Feeling lonely & $\mathbf{1 . 9}$ & $1.21-2.93$ \\
& In the school environment & $\mathbf{1 . 8}$ & $1.02-3.18$ \\
& & & \\
\hline Exercising & Feeling happy & 0.9 & $.51-1.66$ \\
& Feeling satisfied & $\mathbf{2 . 7}$ & $1.47-4.94$ \\
Feeling lively & 1.5 & $.86-2.63$ \\
In the home environment & $\mathbf{0 . 1}$ & $.06-.18$ \\
In the presence of others & $\mathbf{1 . 8}$ & $1.06-3.19$ \\
\hline
\end{tabular}

${ }^{1}$ bold ORs indicate significant findings $(p \leq .05)$

\section{Order Effects of Intrapersonal Factors for Each PA Behavior}

Next, we explored order effects of the PA behaviors associated with intrapersonal factors (i.e., cognitive factors, affective factors, and the perceived environmental factors) across time by means of multilevel time lagged analyses. By calculating this mean, we sought to understand the direction of the relationships between these factors and PA. Table 3 shows associations between (1) factors and the outcome variable at a specific time slots $\left(T_{0}\right),(2)$ factors at a specific time slots and the outcome criterion at the first following time slots $\left(T_{+1}\right)$, and (3) outcome criterion at a specific time slot and factors at the first following time slot $\left(T_{-1}\right)$.

Walking. As can be seen in Table 3, PA intentions ( $O R=2.6,95 \% \mathrm{Cl} 1.70-3.95)$ and $\mathrm{PBC}(\mathrm{OR}=3.2,95 \% \mathrm{Cl}$ 2.13-4.95) were positively associated with walking during a subsequent timeslot. In addition to these cognitive factors, walking was also more likely to occur when students had felt stressed during the 
previous timeslot $(\mathrm{OR}=1.6,95 \% \mathrm{Cl} 1.07-2.41)$. When students felt tired, they were less likely to walk during the following timeslot $(\mathrm{OR}=0.7,95 \% \mathrm{Cl} .47-.99)$. In other words, students were more likely to go for a walk if they had PA intentions, felt in control, or had felt stressed during the previous timeslot, but they were less likely to go for a walk if they felt tired prior to the activity. Moreover, after students had gone for a walk, they were more likely to feel tired $(\mathrm{OR}=1.5,95 \% \mathrm{Cl} 1.05-2.25)$ and less likely to feel lively $(\mathrm{OR}=0.6,95 \% \mathrm{Cl} .42-.94)$.

Cycling. Exploratory time lag analyses showed that PA intentions, PBC, and feeling stressed were positively associated with subsequent cycling $\left(\mathrm{OR}_{\text {intention }}=3.6,95 \% \mathrm{Cl} 2.24-5.68 ; \mathrm{OR}_{\mathrm{PBC}}=4.3,95 \% \mathrm{Cl}\right.$ 2.72-6.92; $\left.\mathrm{OR}_{\text {stressed }}=1.6,95 \% \mathrm{Cl} 1.03-2.46\right)$. Perceived safety was negatively associated with subsequent cycling ( $\mathrm{OR}=0.4,95 \% \mathrm{Cl} .23-.75)$. In turn, cycling was positively associated with subsequent perceptions of safety $(\mathrm{OR}=3.0,95 \% \mathrm{Cl} 1.16-7.73)$ and feelings of boredom $(\mathrm{OR}=1.9,95 \% \mathrm{Cl} 1.24-2.94)$, and negatively associated with subsequent perceptions of the availability of forests $(\mathrm{OR}=0.5,95 \% \mathrm{Cl}$ .28-.99). In other words, students were more likely to cycle if they had PA intentions, felt in control, or had felt stressed during the previous timeslot, but they were less likely to cycle if they felt unsafe prior to the activity. After students had cycled, they were more likely to feel safe and bored, and they perceived forests as less available.

Exercising. Finally, cross-time effects showed that students were more likely to exercise if they had $\mathrm{PA}$ intentions and felt in control during the previous timeslot $\left(\mathrm{OR}_{\text {intention }}=2.7,95 \% \mathrm{Cl} 1.56-4.60 ; \mathrm{OR}_{\mathrm{PBC}}=\right.$ $3.3,95 \% \mathrm{Cl} 1.90-5.70$ ). If they were tired, they were less likely to exercise during the following timeslot $(\mathrm{OR}=0.6,95 \% \mathrm{Cl} .34-.90)$. In other words, students were more likely to go exercise if they had PA intentions and felt in control during the previous timeslot, but they were less likely to exercise if they felt tired prior to the activity. Moreover, after students had exercised, they were more likely to perceive forests as less available $(\mathrm{OR}=0.4,95 \% \mathrm{Cl} .20-.87)$. 
Table 3: Order Effects of Cognitive and Affective Factors for Each PA Behavior ${ }^{1}$

\begin{tabular}{|c|c|c|c|c|c|c|c|c|}
\hline \multirow{3}{*}{ Predictor Variable } & \multicolumn{6}{|c|}{ Walking } & \multirow{3}{*}{$\begin{array}{l}\text { OR } \\
\mathrm{T}+1\end{array}$} & \multirow{3}{*}{$\begin{array}{c}95 \% \mathrm{Cl} \\
\mathrm{T}+1\end{array}$} \\
\hline & OR & $95 \% \mathrm{Cl}$ & OR & $95 \% \mathrm{Cl}$ & OR & $95 \% \mathrm{Cl}$ & & \\
\hline & $\mathrm{T}+1$ & $\mathrm{~T}+1$ & то & то & $\mathrm{T}-1$ & $T-1$ & & \\
\hline Intention & 2.6 & $1.70-3.95$ & 1.2 & $.75-1.79$ & 0.8 & $.53-1.18$ & 3.6 & 2.24-5.68 \\
\hline PBC & 3.2 & 2.13-4.95 & 0.9 & $.57-1.30$ & 1.0 & $.68-1.51$ & 4.3 & 2.72-6.92 \\
\hline Perceived Availability of Forests & 0.5 & $.28-1.06$ & 1.0 & $.56-1.89$ & 0.7 & $.38-1.24$ & 1.3 & $.65-2.55$ \\
\hline Perceived Availability of Parks & 0.7 & $.40-1.09$ & 0.7 & $.40-1.06$ & 1.1 & $.72-1.77$ & 1.4 & $.82-2.37$ \\
\hline Perceived Safety & 0.6 & $.21-1.62$ & 0.9 & $.29-2.99$ & 1.4 & $.75-2.64$ & 0.4 & $.23-.75$ \\
\hline Happy & 0.9 & $.66-1.37$ & 1.5 & $1.05-2.19$ & 0.9 & $.61-1.27$ & 1.0 & $.69-1.54$ \\
\hline Tired & 0.7 & $.47-.99$ & 1.4 & $.92-1.98$ & 1.5 & $1.05-2.25$ & 0.9 & $.59-1.35$ \\
\hline Stressed & 1.6 & $1.07-2.41$ & 1.0 & $.63-1.44$ & 1.3 & $.85-1.87$ & 1.6 & $1.03-2.46$ \\
\hline Bored & 1.0 & $.67-1.47$ & 1.2 & $.82-1.80$ & 1.0 & $.69-1.52$ & 1.2 & $.78-1.83$ \\
\hline Satisfied & 1.1 & $.75-1.56$ & 1.2 & $.80-1.68$ & 0.9 & $.63-1.35$ & 1.1 & $.75-1.71$ \\
\hline Sad & 1.1 & $.70-1.58$ & 1.1 & $.73-1.67$ & 1.0 & $.69-1.56$ & 0.9 & $.59-1.48$ \\
\hline Frustrated & 1.2 & $.77-1.72$ & 0.9 & $.60-1.37$ & 0.8 & $.57-1.27$ & 0.9 & $.58-1.42$ \\
\hline Angry & 0.7 & $.41-1.06$ & 1.1 & $.68-1.74$ & 0.8 & $.49-1.24$ & 0.7 & $.42-1.24$ \\
\hline Lonely & 1.0 & $.70-1.52$ & 1.2 & $.82-1.81$ & 1.0 & $.71-1.54$ & 1.2 & $.80-1.90$ \\
\hline Lively & 1.4 & $.95-2.01$ & 1.7 & $1.14-2.42$ & 0.6 & $.42-.94$ & 1.0 & $.68-1.61$ \\
\hline
\end{tabular}

${ }^{1}$ bold ORs indicate significant findings $(p \leq .05)$ 


\section{Exercising}

\begin{tabular}{|c|c|c|c|c|c|c|c|c|c|}
\hline OR & $95 \% \mathrm{Cl}$ & OR & $95 \% \mathrm{Cl}$ & OR & $95 \% \mathrm{Cl}$ & OR & $95 \% \mathrm{Cl}$ & OR & $95 \% \mathrm{Cl}$ \\
\hline то & то & $\mathrm{T}-1$ & $\mathrm{~T}-1$ & $\mathrm{~T}+1$ & $\mathrm{~T}+1$ & T0 & T0 & $\mathrm{T}-1$ & $\mathrm{~T}-1$ \\
\hline 0.8 & $.50-1.27$ & 0.8 & $.51-1.22$ & 2.7 & $1.56-4.60$ & 0.8 & $.46-1.34$ & 1.1 & $.66-1.85$ \\
\hline 0.7 & $.46-1.15$ & 1.0 & $.65-1.57$ & 3.3 & $1.90-5.70$ & 0.7 & $.39-1.13$ & 1.3 & $.74-2.12$ \\
\hline 0.7 & $.33-1.39$ & 0.5 & $.28-.99$ & 0.9 & $.43-1.85$ & 1.2 & $.59-2.42$ & 0.4 & $.20-.87$ \\
\hline 0.7 & $.33-1.39$ & 1.3 & $.83-2.15$ & 1.2 & $.66-2.12$ & 0.6 & $.35-1.17$ & 1.2 & $.71-2.04$ \\
\hline 0.4 & $.23-.75$ & 3.0 & $1.16-7.73$ & 0.9 & $.25-3.14$ & 2.6 & $.38-17.62$ & 1.5 & $.67-3.47$ \\
\hline 1.5 & $1.01-2.27$ & 0.7 & $.50-1.09$ & 0.8 & $.48-1.25$ & 1.9 & $1.15-3.01$ & 0.9 & $.55-1.43$ \\
\hline 1.1 & $.71-1.66$ & 1.4 & $.94-2.09$ & 0.6 & $.34-.90$ & 1.3 & $.77-2.04$ & 1.3 & $.83-2.15$ \\
\hline 1.1 & $.71-1.75$ & 1.2 & $.81-1.90$ & 1.2 & $.69-2.02$ & 0.9 & $.51-1.51$ & 1.1 & $.64-1.88$ \\
\hline 1.0 & $.68-1.62$ & 1.9 & $1.24-2.94$ & 1.0 & $.60-1.61$ & 0.9 & $.54-1.47$ & 1.0 & $.56-1.63$ \\
\hline 1.2 & $.76-1.76$ & 0.7 & $.49-1.13$ & 0.9 & $.54-1.40$ & 2.7 & $1.65-4.53$ & 0.9 & $.54-1.49$ \\
\hline 1.0 & $.65-1.66$ & 0.8 & $.55-1.31$ & 0.9 & $.50-1.53$ & 1.2 & $.67-1.99$ & 1.1 & $.61-1.84$ \\
\hline 1.1 & $.68-1.67$ & 0.8 & $.52-1.23$ & 0.8 & $.47-1.40$ & 0.7 & $.40-1.21$ & 0.8 & $.48-1.47$ \\
\hline 1.1 & $.63-1.84$ & 1.3 & $.83-1.89$ & 0.7 & $.38-1.40$ & 0.8 & $.42-1.52$ & 0.9 & $.48-1.65$ \\
\hline 1.8 & $1.15-2.75$ & 1.3 & $.83-1.89$ & 1.1 & $.68-1.94$ & 1.3 & $.75-2.20$ & 1.4 & $.84-2.21$ \\
\hline 1.4 & $.91-2.18$ & 1.0 & $.68-1.57$ & 0.7 & $.42-1.18$ & 1.9 & $1.19-3.09$ & 0.7 & $.40-1.15$ \\
\hline
\end{tabular}




\section{Discussion}

The aim of the present study was to understand the relative contribution of motivational (cognitive and affective) and contextual (physical and social environmental) factors on the walking, cycling, and exercising behaviors of emerging young adults within their natural environment (situated in time and place). Affect and (perceived) environmental factors were all expected to be key factors in explaining the inconsistency between previously observed motivational factors and PA (Davidson \& Lawson, 2006; Prins et al., 2009; Prins, 2012; Sallis et al., 1997; Spence \& Lee, 2003). First, findings showed that when emerging young adults reported higher PA intentions and/or feelings of control (Perceived Behavioral Control, PBC), they were more likely to perform subsequent PA behaviors, but that these factors were not associated with PA during the same timeslot. Neither did PA show cross-time effects on subsequent PA intentions and PBC. Affective factors showed associations with PA behaviors across time. For example, when emerging young adults felt stressed, they were more likely to cycle during the following timeslot $\left(T_{+1}\right)$, when they walked they were also more likely to feel happy $\left(T_{0}\right)$, and they were more likely to feel tired after they had walked $\left(T_{-1}\right)$. Finally, with regard to environmental factors, multivariate models showed that PA was less likely to occur in the home environment, and only exercising was more likely to occur when others were present (instead of when the participant was alone).

Overall, the assessment of cognitive, affective and environmental factors in a singular mEMA study, and the use of both multivariate and time lagged analyses both extended and confirmed some previous findings. First, the exploratory cross-time associations found between cognitive factors and PA support many motivational PA models, showing that intentions and PBC are associated with subsequent PA behaviors, or in other words, that reasoned decision making underlies PA behaviors (Ajzen, 2011; McEachan, Conner, Taylor, \& Lawton, 2011). Second, affect was associated with PA behaviors across time, indicating that the two are interrelated. However, it should be noted that we only found an association between positive affect (e.g., happiness) and PA within a particular timeslot, and not over time. This is in contrast with previous studies indicating an interrelationship between the two (Ajzen, 2011; Motl, Birnbaum, Kubik, \& Dishman, 2004; Penedo \& Dahn, 2005). Albeit the difference in targeted populations (e.g., Motl. et al. (2004) investigated PA in adolescents), other methodological differences might also account for this disparity. We used mEMA rather than surveys to investigate the socio-ecological model proposed in the present study, unlike the studies included in the review by Penedo and Dahn (2005). The repeated assessment of behaviors and determinants in real-time 
and real-world settings arguably increase the accuracy of these measures (Haedt-Matt \& Keel, 2011; Stone et al., 2007). This increase in accuracy is of considerable importance in reducing limitations that are associated with retrospective self-report, such as memory limitations and cognitive biases (Haedt-Matt \& Keel, 2011). Subsequently, overestimation of PA is less likely using these methods as compared to retrospective self-reports, as recall periods are shorter and social desirability effects are less likely to occur (Adams et al., 2005). The frequency (and duration) of reported PA behaviors in the present study were, therefore, expected to be more precise as compared to previously self-reported PA frequencies. In fact, in the present study, 39\% of emerging young adults reported that they had been physically active. This is lower than the $56 \%$ of youth who previously self-reported that they had reached the Dutch PA norms (aged 15-28 years; Rijpstra \& Bernaards, 2011). As this 39\% does not take into account the duration of PA, the true percentage of emerging young adults that reach the Dutch PA norms (i.e., 30 minutes of moderate vigorous physical activity at least five days a week, and/ or at least 20 minutes of vigorous physical activity three times a week) may be even lower.

\section{Conclusion}

The present mobile-based Ecological Momentary Assessment (mEMA) study targeting determinants of physical activity (PA) among emerging young adults showed that intention and perceived behavioral control (PBC) determine subsequent PA behavior, but that these cognitive factors are not associated with PA during the same timeslot $\left(T_{0}\right)$. As such, these fluctuations over time may explain the differences in relationships between cognitions and PA behaviors as shown in previous cross-sectional studies. 


\section{List of abbreviations}

mEMA, mobile-based Ecological MomentaryAssessment; PA, Physical Activity; OR, Odds Ratio; Cl, Coincidence Interval; PBC, Perceived Behavioral Control; GLMM, General Linear Mixed Models; M, Mean; SD, Standard Deviation; Mdn, Median; BMI, Body Mass Index.

\section{Competing interests}

The authors declare that they have no competing interests.

\section{Author's contributions}

JS conceptualized and designed the study, carried out the mEMA study, performed the initial statistical analyses, and drafted the initial manuscript, and approved the final manuscript as submitted. PvE conceptualized and designed the study, reviewed and revised the manuscript, and approved the final manuscript as submitted. TP and GK conceptualized and designed the study, reviewed and revised the manuscript, and approved the final manuscript as submitted. All authors read and approved the final manuscript. 


\section{References}

Adams, S.A., Matthews, C.E., Ebbeling, C.B., Moore, C.G., Cunningham, J.E., Fulton, J., et al., 2005. The Effect of Social Desirability and Social Approval on Self-Reports of Physical Activity. American Journal of Epidemiology 161(4), 390-398.

Ajzen, I., 1991. The theory of planned behavior. Organizational Behavior and Human Decision Processes 50, 179-211. doi:10.1016/0749-5978(91)90020-T

Ajzen, I., 2011. The theory of planned behaviour: reactions and reflections. Psychology \& Health. 26(9), 1113-27.

Andersen, R.E., Crespo, C.K., Bartlett, S.J., Cheskin, L.J., Pratt, M., 1998. Relationship between physical activity and television watching with body weight and level of fatness among children. JAMA 279(12), 938-42.

Arnett, A.J., 2000. Emerging adulthood: A theory of development from the late teens through the twenties. American Psychologist 55(5), 469-80.

Bernaards C., van Buuren, S., 2012. Rapportage veranderingen in het beweeggedrag van mbo studenten. Leiden, the Netherlands: TNO Behavioural and Societal Sciences Report No.: R10185.

Boreham C., Riddoch C., 2001. The physical activity, fitness and health of children. Journal of Sports Science $19,915-29$.

Broderick, J.E., Schwartz, J.E., Shiffman, S., Hufford, M.R., Stone, A.A., 2003. Signaling does not adequately improve diary compliance. Annals of Behavioral Medicine 26, 139-48.

Carels, R.A., Hoffman, J., Collins, A., Raber, A.C., Cacciapaglia, H., O'Brien, W.H., 2001. Ecological momentary assessment of temptation and lapse in dieting. Eating Behaviors 2, 307-21.

Caspersen C.J., Pereira, M.A., Curran, K.M., 2000. Changes in physical activity patterns in the United States, by sex and cross-sectional age. Medicine \& Science in Sports \& Exercise 32, 1601-9.

Davidson, K.K., Lawson, C., 2006. Do attributes in the physical environment influence children's physical activity? A review of the literature. International Journal of Behavioral Nutrition and Physical Activity 3(19). 
Dunton, .GF., Liao, Y., Intille, S.S., Spruijt-Metz, D., Pentz, M., 2011. Investigating Children's Phychical Activity and Sedentary Behavior Using Ecological Momentary Assessment with Mobile Phones. Obesity 19, 1206-1212.

Dunton, G.F., Whalen, C.K., Jamner, L.D., Floro, J.N., 2007. Mapping the social and physical contexts of physical activity across adolescence using ecological momentary assessment. Behavioral Medicine 34(2), 144-53.

Dunton, G.F., Whalen, C.K., Jamner, L.D., Henker, B., Floro, J.N., 2005. Using Ecologic Momentary Assessment to Measure Physical Activity During Adolescence. Am J Prev Med. 29(4), 281-7.

Ekkekakis, P., Petruzzello, S.J., 2000. Analysis of the affect measurement conundrum in exercise psychology. Psychology of Sport and Exercise 1, 71-88.

Haedt-Matt, A.A., Keel, P.K., 2011. Revisiting the affect regulation model of binge eating: a meta-analysis of studies using ecological momentary assessment. Psychological Bulletin 137(4), 660-81.

Hagger, M.S., Chatzisarantis, N.L.D., Biddle, S.J.H., 2002. A meta-analytic review of the theories of reasoned action and planned behavior in physical activity: Predictive validity and the contribution of additional variables. Journal of Sport and Exercise Psychology 24, 3-32.

Healthy People. Physical Activity and Fitness. 2010. Downloaded from http://web.health.gov/healthypeople/document/HTML/Volume2/22Physical.htm [Downlaoded at 23th June, 2015].

McEachan, R.R.C., Conner, M., Taylor, N., Lawton, R.J., 2011. Prospective prediction of health-related behaviors with the Theory of Planned Behavior: A meta-analysis. Health Psychology Review 5, 97-144.

Motl, R.W., Birnbaum, A.S., Kubik, M.Y., Dishman, R.K., 2004. Naturally occurring changes in physical activity are inversely related to depressive symptoms during early adolescence. Psychosomatic Medicine 66(3), 336-42.

Motl, R.W., Dishman, R.K., Saunders, R., Dowda, M., Felton, G., Ward, D.S., et al., 2002. Examining social-cognitive determinants of intention and physical activity among black and white adolescent girls using structural equation modeling. Health Psychology 21, 459-67.

Penedo, F.J., Dahn, J.R.., 2005. Exercise and well-being: A review of mental and physical health benefits associated with physical activity. Current Opinion in Psychiatry 18(2), 189-93. 
Popkin, B.M., Duffey, K., Gordon-Larsen, P., 2005. Environmental influences on food choice, physical activity and energy balance. Physiology \& Behavior 86(5), 603-13.

Prins, R.G., 2012. Environmental influences on physical activity among adolescents [Dissertation]. Rotterdam: Erasmus Universiteit Rotterdam.

Prins, R.G., van Empelen, P., Beenackers, M.A., Brug, J., Oenema, A., 2010. Systematic development of the YouRAction program, a computer-tailored physical activity promotion intervention for Dutch adolescents, targeting personal motivations and environmental opportunities. BMC Public Health 10, 474.

Prins, R.G., Kamphuis, C.B.M., van Empelen, P., Beenackers, M.A., Brug, J., Mackenbach, J.P., et al., 2013. Explaining socio-demographic differences in disengagement from sports in adolescence. European Journal of Public Health 23(5), 811-6.

Prins, R.G., Oenema, A., van der Horst, K., Brug, J., 2009. Objective and perceived availability of physical activity opportunities: differences in associations with physical activity behavior among urban adolescents. International Journal of Behavioral Nutrition and Physical Activity 6, 70.

Rijpstra, A., Bernaards, C., 2005. De leefstijl van MBO studenten in Nederland 2009/2010. Leiden, the Netherlands: TNO; 2011. Report No.: TNO/LS 2011.014.21. Romero AJ. Low-income neighborhood barriers and resources for adolescents' physical activity. Journal of Adolescent Health 36, 253-9.

Ronda, G., van Assema, P., Brug, J., 2001. Stages of change, psychological factors and awareness of physical activity levels in the Netherlands. Health Promotion International 16(4), 305-14.

Rovniak, L.S., Anderson, E.S., Winett, R.A., Stephans, R.S., 2002. Social cognitive determinants of physical activity in young adults: A prospective structural equation analysis. Annual Behavioral Medicine 24(2), 149-56.

Sallis, J.F., Johnson, M., Calfas, K., Caparosa, S., Nichols, J., 1997. Assessing the perceived physical environmental variables that may influence physical activity. Research Quarterly for Exercise and Sport $68,345-51$.

Sallis, J.F., Owen, N., 2002. Ecological models of health behavior. In: Glanz, K., Rimer, B.K., Marcus, L.F., (Eds), Health behavior and health education. San Fransisco: Jossey-Bass, pp. 462-84. 
Spence, J.C., Lee, R.E., 2003. Toward a comprehensive model of physical activity. Psychology of Sport and Exercise 4, 7-24.

Spook, J.E., Paulussen, T., Kok, G., van Empelen, P., 2013. Monitoring dietary intake and physical activity electronically: feasibility, usability, and sensitivity of a mobile-based Ecological Momentary Assessment (mEMA) tool. Journal of Medical Internet Research 15(9), e214.

Spook, J.E., Paulussen, T., Kok, G., van Empelen, P., submitted for publication. The role of motivational and contextual factors on intentional and craving-induced snack consumption: A mobile-based Ecological Momentary Assessment study.

Stone, A.A., Shiffman, S., de Vries, M.W., 2000. Ecological Momentary Assessment. In: Kahneman, D., Diener, E., Schwarz, N., (Eds), Well-being: the foundations of hedonic psychology. New York: Russell Sage Foundation, pp. 26-39.

Stone, A.A., Shiffman, S., Atienza, A.A., Nebeling, L., 2007. The science of real-time data capture. Self-reports in health research. New York: Oxford University Press.

Telama, R., Yang, X., Viikari, J., Valimaki, I., Wanne, O., Raitakari, O., 2005. Physical activity from childhood to adulthood: A 21-year tracking study. American Journal of Preventive Medicine 28, 267-73.

The European Heart Initiative. Children and young people - the importance of physical activity. 2001. http:/ /ec.europa.eu/health/ph_projects/2000/promotion/fp_promotion_2000_frep_11_en.pdf 



\section{CHAPTER 5}

Design Rationale Behind the Serious Self-Regulation Game Intervention

"Balance It": Overweight Prevention Among Secondary Vocational

Education Students in The Netherlands

Published: Spook, J. E., Paulussen, T., Kok, G., \& van Empelen, P.

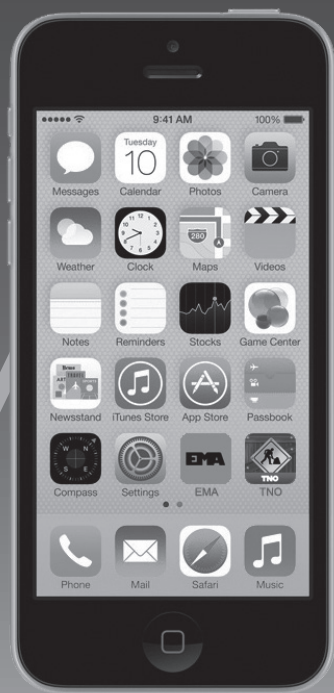

intervention "balance it": Overweight prevention among secondary

vocational education students in the Netherlands. Games for Health

Journal: Research, Development, and Clinical Applications, 4(6) 


\title{
Design Rationale Behind the Serious Self-Regulation Game Intervention "Balance It": Overweight Prevention Among Secondary Vocational Education Students in The Netherlands
}

\begin{abstract}
Objective: This article describes the design rationale behind a serious self-regulation game intervention. The aim of the game is to promote healthy dietary intake and physical activity among secondary vocational educational students in The Netherlands (approximately 16-20 years of age).

Materials and Methods: The game "Balance It" was developed according to the intervention mapping (IM) protocol. Following IM, we specified health promotion and usability objectives and linked these objectives to selected behavior change and persuasive methods. Accordingly, these methods were translated into a coherent intervention program.

Results: The health behavior change objectives were derived from the determinants of the behavior and from Self-Regulation Theory (e.g., students are asked to set goals, monitor and evaluate their behavior, and create coping plans). Usability objectives were derived from the RE-AIM model (i.e., Reach, Effectiveness, Adoption, Implementation, and Maintenance). Next, behavior change and persuasive techniques were selected (e.g., goal setting and prompting, respectively) that did fit with the targeted change objectives and were translated in the design of a (mobile) serious self-regulation game intervention. Subsequently, three concept and usability tests were performed to improve intervention usability, and an evaluation plan was developed.

Conclusion: The aim of this study was to provide a design rationale for game interventions targeting health-related behaviors. We developed a coherent program design in which both health behavior change and usability factors are addressed. The IM protocol can serve as a useful guide for decision making in program development and evaluation.
\end{abstract}

Keywords: Serious Self-Regulation Game; Intervention Mapping; Design Rationale; Overweight Prevention 


\section{Introduction}

One of the largest health concerns in Western countries is the prevalence of overweight and obesity, especially among youth from low socioeconomic (SES) families and minority groups (Bernaards \& Buuren van, 2012; Wang et al., 2007). In The Netherlands, being overweight is especially prevalent among low SES youth and youth from minority groups (Bernaards \& Buuren van, 2012; Schönbeck \& van Buuren, 2010). Overweight percentages among youth vary between 16 percent (Dutch origin) to 34 percent (Moroccan origin) to 40 percent (Turkish origin) (Schönbeck \& van Buuren, 2010), and about $20 \%$ of low SES youth in general (16-20 years of age) is overweight or obese (Bernaards \& Buuren van, 2012). Yet these percentages are still rising (Schönbeck \& van Buuren, 2010), along with the health risks associated with being overweight or obese.

There are various short- and long-term health-related and financial consequences associated with being overweight or obese, including cardiovascular disease, diabetes, musculoskeletal disorders, and cancer (Polder \& Takkern, 2002; World Health Organization, 2013). Moreover, being overweight or obese during adolescence is a strong predictor of being overweight or obese during adulthood, and yet the effectiveness of treatment of adult overweight and obesity is limited (Maffeis \& Tato, 2001; Must, 2003; Seidell, 2000; van Genugten, 2011). Bearing in mind that being overweight is generally the result of an energy imbalance, whereby energy intake is higher than energy expenditure (Kumanyika et al., 2008), our main focus was to improve dietary intake (DI) (fruit, vegetable, snack, and soft drink consumption) and increase physical activity (PA) among low SES youth in The Netherlands.

Previous evaluation studies have shown that the effects of electronic health (e-health) interventions, targeting PA and/or DI, are generally small and are hampered by poor intervention use and large dropout rates (Crutzen, Kohl, \& de Vries, 2013). Serious gaming may be a means to increase the persuasive appeal, engagement, and reward of the (sustained) use of an intervention (DeSmet et al., 2014). Serious games are visually attractive, are highly interactive, and enable digital gamebased learning (DeSmet et al., 2013; Prensky, 2007). These games may also promote learning, increase personal relevance, promote mastery learning of challenging but feasible goals, and increase interactivity (DeSmet et al., 2014; Kreps \& Neuhauser, 2010; World Health Organization, 1998). Young (low SES) people are often active gamers and smartphone users (Lu, Baranowski, Thompson, \& Buday, 2012; Spook, Paulussen, Kok, \& van Empelen, 2013), and as such, serious gaming might be suitable for reaching at-risk youth, who are generally difficult to reach and often show poor compliance with 
intervention programs (Crutzen et al., 2013). As low SES adolescents in The Netherlands often participate in secondary vocational education (Ministery of Education, Culture, and Science, 2012), the smartphone-based serious self-regulation game intervention "Balance It" was targeted at secondary vocational education students (16-20 years of age).

This article describes the rationale behind the systematic development of "Balance It." "Balance It" was designed to be implemented within the context of an existing student tracking system called “Lifestyle Reality Check" (in Dutch, Test Je Leefstijl [TJL]) (Bernaards, van Keulen, Rijpstra, \& Paulussen, 2011). TJL currently provides tailored feedback based on students' self-reported DI and PA, but TJL only provides behavioral feedback and references to interventions. We developed "Balance It" to promote student engagement and to provide support to actively change their DI and/or PA. In order to ensure a solid theoretical and empirical foundation underlying the game-based intervention, we used a protocol to systematically plan evidence-based health interventions, called intervention mapping (IM) (Bartholomew, Parcel, Kok, Gottlieb, \& Fernández, 2011).

\section{Methods}

In order to maximize the effectiveness and usability of the intervention, appropriate consideration and application of theory and empirical evidence are required in the selection of intervention objectives and methods. The IM protocol describes the iterative path from problem identification to problem solving (Bartholomew et al., 2011). IM contains six steps, and each step comprises several theory- and evidence-based tasks. The completion of the tasks in each step creates a product that is the guide for the subsequent step. The completion of all of the steps serves as a blueprint for designing, implementing, and evaluating an intervention that is based on a foundation of theoretical, empirical, and practical information. The six steps of the IM protocol (and the tasks related to each of them) are as follows: 1) Conduct a needs assessment to identify the health problem, the behavioral and environmental causes of this problem, and related determinants; 2) Create matrices of change objectives, specifying what the target population needs to learn or change in order to reach the overall (behavioral) goal of the intervention. These change objectives are the product of (sub-) behaviors (i.e., the so-called performance objectives) and determinants, the factors that mediate the changes in performance objectives; 3) Select theory-based intervention methods (i.e., behavior change techniques [BCTs] and persuasive techniques [PTs]) that match the determinants into which the identified health performance and usability objectives aggregate and translate these into prac- 
tical applications that satisfy the requirements for effectiveness of the selected methods; 4) Integrate methods and practical applications into a coherent intervention program (e.g., game mechanics); 5) Plan for the adoption, implementation, and sustainability of the intervention in the real-life contexts to ensure program use, paying attention to usability factors (e.g., sending reminders) and program implementation factors (e.g., embedding the intervention in an existing structure); 6) Generate a plan to evaluate the procedure and the effects of the intervention (in this case, the game "Balance It"). To clarify the IM process, all steps are visualized in Figure 1, and definitions of all concepts can be found in the Appendix.

Figure 1: Design rationale

\begin{tabular}{|c|c|c|c|}
\hline & IM Step & Health Promotion Goal & Usability Goal \\
\hline \multirow{8}{*}{$\uparrow$} & 1 & \multicolumn{2}{|c|}{ Needs Assessment } \\
\hline & \multirow[b]{2}{*}{2} & \multicolumn{2}{|c|}{ Matrices } \\
\hline & & $\begin{array}{l}\text { Performance Objectives - Health } \\
\text { Determinants - Health } \\
\text { Change Objectives - Health }\end{array}$ & $\begin{array}{c}\text { Performance Objectives - Usability } \\
\text { Determinants - Usability } \\
\text { Change Objectives - Usability }\end{array}$ \\
\hline & \multirow{2}{*}{3} & \multicolumn{2}{|c|}{ Methods \& Practical Applications } \\
\hline & & Behavior Change Techniques & Persuasive Techniques \\
\hline & \multirow[b]{2}{*}{4} & \multicolumn{2}{|c|}{ Concept Intervention Program } \\
\hline & & \multicolumn{2}{|c|}{$\begin{array}{c}\text { Game Genre \& Concept } \\
\text { Game Elements } \\
\text { Game Process } \\
\text { Concept and Usability Testing }\end{array}$} \\
\hline & 5 & \multicolumn{2}{|c|}{ Program Adoption and Implementation Plan } \\
\hline 4 & 6 & \multicolumn{2}{|c|}{ Evaluation Plan } \\
\hline
\end{tabular}

It is important to note that the systematic development of a serious game intervention entails the consideration of both health performance-related goals and usability goals. This is because both behavior change and intervention use are mutually important in such interventions. In the present article, our aim is to illustrate how this process resulted in the development of a serious game called "Balance It" and how this can be embedded within an existing system (e.g., the current student tracking system of TJL). 


\section{Results}

\section{IM Step 1: needs assessment}

Following IM Step 1, we established a needs assessment in order to specify the health problem, the behavioral and environmental causes of this problem, and the determinants of these causes. About 20 percent of secondary vocational education students in The Netherlands are overweight or obese (Bernaards \& Buuren van, 2012; Ministery of Education, Culture, and Science, 2012), meaning approximately 106,000 students in 2010-2011. Students enrolled in vocational education are generally at risk for being/becoming overweight, as a majority of these students come from low SES families or ethnic minority groups with a higher prevalence of overweight and obesity (e.g., Turkish and Moroccan youth; (Schönbeck \& van Buuren, 2010)). Considerable numbers of secondary vocational education students have an unhealthy eating pattern and do not reach the recommended PA norms (Bernaards \& Buuren van, 2012). In a previous mobile-based ecological momentary assessment study, we found that snack consumption among these students was more likely to occur at the end of the day or when experiencing cravings (Spook, Paulussen, Kok, \& van Empelen, submitted for publication). It has also been shown that these students are more likely to engage in PAs when they have PA intentions and feel capable of executing the specific behavior, as well as do sports mostly together with their peers (Spook, Paulussen, Kok, \& van Empelen, submitted for publication).

\section{IM Step 2: matrices of change objectives}

IM Step 2 specifies who and what will change as a result of the intervention (Bartholomew et al., 2011). The overall health-related goal was to prevent overweight by improving DI and increasing PA among secondary vocational education students. The overall usability goal was to optimize initial and continued use of the intervention.

Performance objectives for health. Performance objectives for health were derived from Self-Regulation Theory (Zeidner, Boekaerts, \& Pintrich, 2000). Self-Regulation Theory provides the basis of purposeful action and describes subbehaviors that are necessary to initiate, continue, and maintain both a healthy diet and a healthy level of PA. These subbehaviors can be categorized as goal establishment, goal setting, goal planning, goal striving, goal revision, and maintenance (Maes \& Karoly, 2005; van Genugten, van Empelen, Flink, \& Oenema, 2010). It has been shown that individuals with good self-regulation skills are more successful in changing their DI and PA (Chambers \& Swanson, 
2012; Schnoll \& Zimmerman, 2001; van Genugten, 2011). Moreover, self-regulatory skills are key to decreasing the risk of regaining weight after weight loss and to maintaining a healthy diet (Wing, Tate, Gorin, Raynor, \& Fava, 2006). Nine performance objectives for health were specified for both DI and PA program objectives (Table 1); of these, the goal establishment objectives ( $1 A$ and $B$ ) were already covered within the present student tracking system of TJL. As such, the main focus of our intervention was on goal setting, planning, striving, revision, and maintenance (i.e., referring to the self-regulation subbehaviors). However, without initial motivation to change behavior and to actually use the intervention, there is slim chance for success (Crutzen et al., 2013; Ryan \& Deci, 2000). Therefore, we focused on performance objectives for usability and for health simultaneously.

Performance objectives for usability. Performance objectives for usability were derived from the RE-AIM model proposed by Glasgow et al. (Glasgow, Vogt, \& Boles, 1999). RE-AIM is an acronym for Reach, Effectiveness, Adoption, Implementation, and Maintenance, referring to the key factors that contribute to the public health impact of an intervention. As smartphone-delivered interventions can be used in a stand-alone fashion, adoption, implementation, and maintenance occur at the individual level (Crutzen et al., 2008; Crutzen et al., 2009; Crutzen et al., 2013). In relation to our game, this means that individuals can decide for themselves if they want to start playing "Balance It," if they want to continue playing the game as intended, and if they want to maintain use of "Balance It." In order to encourage the target population to start using the intervention, both motivation for behavior change and curiosity regarding the content of the intervention play important roles (Brouwer et al., 2009; Crutzen et al., 2013). Based on these usability factors of the RE-AIM model, we specified as performance objectives that the target population should (1) be aware of the intervention, (2) accept the intervention, (3) use the intervention as intended, and (4) use the intervention over a prolonged period of time. Seven performance objectives for usability are specified and reported in Table 1. 
Table 1: Performance Objectives based on self-regulation theory and the RE-AIM model

\begin{tabular}{|c|c|c|}
\hline \multicolumn{3}{|c|}{ Performance Objectives } \\
\hline Dietary Intake (DI) & Physical Activity (PA) & Usability \\
\hline $\begin{array}{l}\text { 1. GOAL ESTABLISHMENT: } \\
\text { A. Students decide to maintain a } \\
\text { healthy weight } \\
\text { B. Students decide to improve } \\
\text { their DI } \\
\text { I) Eat more vegetables } \\
\text { II) Eat more fruit } \\
\text { III) Consume less snacks }\end{array}$ & $\begin{array}{l}\text { 1. GOAL ESTABLISHMENT: } \\
\text { A. Students decide to maintain a } \\
\text { healthy weight } \\
\text { B. Students decide to improve } \\
\text { their PA } \\
\text { I) Walk more (often) } \\
\text { II) Bike more (often) } \\
\text { III) Exercise more (often) }\end{array}$ & $\begin{array}{l}\text { 1. REACH: } \\
\text { Students become familiar with } \\
\text { the intervention }\end{array}$ \\
\hline $\begin{array}{l}\text { 2. GOAL SETTING: } \\
\text { A. Students set a specific, } \\
\text { achievable goal to change } \\
\text { their DI } \\
\text { B. Students select strategies to } \\
\text { change their DI: } \\
\text { I) Avoid possible snacks } \\
\text { II) Reduce amount of snacks } \\
\text { III) Substitute (caloric) snacks } \\
\text { for healthier alternatives }\end{array}$ & $\begin{array}{l}\text { 2. GOAL SETTING: } \\
\text { A. Students set a specific, } \\
\text { achievable goal to increase } \\
\text { PA } \\
\text { B. Students select strategies to } \\
\text { increase PA } \\
\text { I) Increase PA duration } \\
\text { II) Increase PA frequency } \\
\text { III) Substitute "passive" } \\
\text { modes of transport for } \\
\text { active transport }\end{array}$ & $\begin{array}{l}\text { 2. ADOPTION: } \\
\text { Students adopt the intervention }\end{array}$ \\
\hline
\end{tabular}

3. GOAL PLANNING:

Students plan when, where, and how to achieve their selected strategy (see GOAL SETTING 2B, I $\mathrm{t} / \mathrm{m}$ III)
3. GOAL PLANNING:

Students plan when, where, and how to achieve their selected strategy (see GOAL SETTING 2B, I t/m III)

\section{IMPLEMENTATION:}

A. Students use the intervention as intended

B. Students keep using the intervention

C. Implementers implement the intervention correctly in order to realize the effectiveness of the intervention

4. MAINTENANCE:

4. GOAL STRIVING:

Students perform the selected strategy (see GOAL SETTING 2B, I t/m III)
Students perform the selected strategy (see GOAL SETTING 2B, $\mathrm{It} / \mathrm{m}$ III)

\section{A. Students frequently use the intervention over a longer period of time \\ B. Students maintain interven-} tion usage
5. GOAL REVISION \& MAINTENANCE:

A. Students anticipate situations that are likely to be challenging (and create coping strategies)

B. Students monitor and evaluate their own DI and goal progress

C. Depending on goal progression (failure):

I) They adapt their goal

II) They maintain their goal

III) They start with a new goal
5. GOAL REVISION \& MAINTENANCE:

A. Students anticipate situations that are likely to be challenging (and create coping strategies)

B. Students monitor and evaluate their PA and goal progress

C. Depending on goal progression (failure):

I) They adapt their goal

II) They maintain their goal

III) They start with a new goal 
Determinants. The most relevant and changeable determinants concerning DI and PA (i.e., health determinants), and intervention implementation (i.e., implementation determinants) were selected based on a review of the literature and mobile-based ecological momentary assessment studies (Spook et al., submitted for publication).

Determinants targeting health behavioral outcomes. In order to achieve the health performance objectives, determinants were derived from health promotion theories, such as Self-regulation Theory (Zeidner et al., 2000), the Theory of Planned Behavior (Ajzen, 1991), the Precaution Adoption Process Model (Weinstein \& Sandman, 2002), implementation intentions and goal setting (Gollwitzer \& Sheeran, 2006), Relapse Prevention Theory (Marlatt \& Gordon, 1985), and Self-Determination Theory (Ryan \& Deci, 2000). The following determinants were included in "Balance It": attitude, awareness, self-efficacy, skills, perceived barriers, and social support. The selection of these determinants was based on consideration of the aforementioned theories as well as empirical research on overweight prevention (Adriaanse, Ridder de, \& Wit de, 2009; Kremers et al., 2006; van Genugten et al., 2010). We also took into account the findings of Prins (Prins, 2012), who highlighted the importance of perceived physical opportunities for behavior engagement. He found that the stronger the link was between perceived and actual PA opportunities, the more likely young people were to act on their intention to engage in PA activities. Hence, increased awareness of opportunities is likely to result in the actual uptake of the behavior. In relation to our game, opportunities for DI and PA were derived from ecological assessment studies, in which we identified potential physical, social, and motivational triggers (Spook et al., 2013; Spook et al., submitted for publication; Spook et al., submitted for publication)

Determinants targeting usability. The selection process regarding determinants of usability was also based on theory and empirical evidence. An examination of possible usability factors showed that three types of determinants should be taken into account when considering serious game design: motivation, ability (simplicity) and effective triggers of behavior (see the behavior model proposed by Fogg (Fogg, 2009)). For the behavior to occur, all three factors need to be sufficiently present. This means that when the player perceives the game as simple but challenging enough (or highly motivating), the player will be triggered to execute the desired behavior (i.e., game use) (Fogg, 2009). This is in line with the need for competence and autonomy derived from Self-Determination Theory (Ryan \& Deci, 2000). Together with the need for relatedness, stressing the importance of social support, these three basic needs improve internal motivation to perform behavior. 
Change objectives. Finally, change objectives for health and usability were defined based on the combination of performance objectives and the determinants that were selected. Examples of the change objectives for health and change objectives for usability can be found in Tables 2 and 3 .

Table 2: Examples of Change Objectives for Health

\begin{tabular}{llll}
\hline $\begin{array}{l}\text { Determinants } \rightarrow \\
\downarrow \text { Performance Objective }\end{array}$ & Awareness & Self-efficacy & Skills \\
\hline $\begin{array}{l}\text { Students set a specific, } \\
\text { achievable goal with } \\
\text { regard to changing their DI }\end{array}$ & $\begin{array}{l}\text { AW1: they give reasons as } \\
\text { to why setting specific, } \\
\text { challenging, achievable } \\
\text { goals is important }\end{array}$ & $\begin{array}{l}\text { SE1: they express } \\
\text { confidence that they can } \\
\text { set goals }\end{array}$ & $\begin{array}{l}\text { SK1: they demonstrate the } \\
\text { ability to set goals }\end{array}$ \\
& & $\begin{array}{l}\text { SK2: they demonstrate the } \\
\text { ability to estimate whether } \\
\text { a goal is achievable or not }\end{array}$
\end{tabular}

Table 3: Examples of Change Objectives for Usability

\begin{tabular}{llll}
\hline \multirow{2}{*}{$\begin{array}{c}\text { Determinants } \rightarrow \\
\downarrow \text { Performance Objective }\end{array}$} & \multicolumn{2}{c}{ Usability } \\
\cline { 2 - 4 } $\begin{array}{l}\text { Students keep using the } \\
\text { intervention }\end{array}$ & $\begin{array}{l}\text { M1: they evaluate the } \\
\text { intervention as engaging }\end{array}$ & $\begin{array}{l}\text { A1: they return to the } \\
\text { intervention multiple } \\
\text { times }\end{array}$ & $\begin{array}{l}\text { T1: they report that they } \\
\text { receive prompts }\end{array}$ \\
& $\begin{array}{l}\text { M2: they experience feel- } \\
\text { ings of mastery }\end{array}$ & $\begin{array}{l}\text { T2: they report that they } \\
\text { programmed prompts } \\
\text { themselves }\end{array}$ \\
& $\begin{array}{l}\text { M3: they express feelings } \\
\text { of control }\end{array}$ & \\
\hline
\end{tabular}

\section{IM Step 3: methods and practical applications}

Having specified the change objectives in terms of what needed to be achieved in the self-regulation game, BCTs and PTs were selected that would facilitate these changes.

BCTs. When considering effective BCTs, we relied on two approaches: a selection of methods related to the targeted determinants and a selection of techniques found to be effective in changing DI or PA behavior. Based on a meta-analytic review that assessed effective combinations of BCTs in e-health interventions to increase levels of DI or PA (Dusseldorp, van Genugten, van Buuren, Verheijden, \& van Empelen, 2013), it was concluded that the following combinations of BCTs were most effective in changing DI or PA: (1) information about health-related behaviors with prompt intention formation and (2) information about health-related behaviors combined with information about their consequences and the use of follow-up prompts. These BCTs did fit with the selected determinants 
and health performance objectives. Interventions that provide information on performance without providing instructions were the least effective. In addition, alternative theoretical methods were selected that targeted a specific determinant (Bartholomew et al., 2011). For each of the methods that we selected, parameters for use (i.e., the conditions under which the theoretical method will be effective) were identified, in order to ensure the effective translation of a BCT into its practical application (Bartholomew et al., 2011). Table 4 provides an example of the selection of a technique, identification of the parameters of use, and their application within the game.

Table 4: Example of the Translation of a Change Objective for Health into Its Practical Application

\begin{tabular}{|c|c|c|c|c|c|}
\hline $\begin{array}{l}\text { Performance } \\
\text { Objective }\end{array}$ & $\begin{array}{l}\text { Change } \\
\text { Objective }\end{array}$ & Determinant & $\begin{array}{l}\text { Behavior } \\
\text { Change } \\
\text { Technique }\end{array}$ & Parameter for Use & Application \\
\hline $\begin{array}{l}\text { Students set } \\
\text { a specific, } \\
\text { achievable } \\
\text { goal in order to } \\
\text { change their DI }\end{array}$ & $\begin{array}{l}\text { They } \\
\text { demonstrate } \\
\text { the ability to } \\
\text { set goals }\end{array}$ & Skills & Goal setting & $\begin{array}{l}\text { Commitment to } \\
\text { the goal; goals } \\
\text { that are difficult } \\
\text { but achievable } \\
\text { considering } \\
\text { the individual's } \\
\text { skill level } \\
\text { (Bartholomew et } \\
\text { al., 2011) }\end{array}$ & $\begin{array}{l}\text { Students set } \\
\text { specific goals within } \\
\text { the intervention. } \\
\text { Commitment is } \\
\text { established through } \\
\text { game-engaging } \\
\text { elements (e.g., } \\
\text { triggers). } \\
\text { New goals could be } \\
\text { set (if not challenging) }\end{array}$ \\
\hline
\end{tabular}

In addition, we selected BCTs aimed at promoting actual use of the self-regulation game intervention. These BCTs were derived from the taxonomy of Abraham and Michie and mapped onto Self-Determination Theory (Abraham \& Michie, 2008; Ryan \& Deci, 2000). BCTs that were selected to enhance the need for competence encompassed prompt practice, self-monitoring of behavior, and the provision of feedback on performance (e.g., "You did it!" [repeats the goal that was set by the student], "How are you feeling?"). To create feelings of relatedness, the present self-regulation intervention includes a social support system enabling players to provide and receive social support from peers. The possibility of playing the game together with peers might further increase feelings of relatedness. Finally, to facilitate users' need for autonomy, the self-regulation game intervention encourages players to set graded tasks, providing them with the opportunity to decide for themselves how they want to work on DI or PA. In addition to motivational purposes, our intervention provides instructions about how the game should be played (i.e., provided instructions) and prompts players to trigger intervention usage (i.e., prompted practice). In addition to BCTs, PTs have been associated with an increase in intervention effectiveness (Fogg, 2009). 
PTs. PTs were derived from Fogg (2009), Fogg et al. (2002), and DeSmet et al. (2013) and mapped onto the usability objectives and usability determinants (DeSmet et al., 2013; Fogg, Grudin, Nielsen, \& Card, 2002; Fogg, 2009). In general, models for persuasive game design focus on motivation, ability (simplicity), and effective triggers for behavior (Fogg, 2009). These factors are described as usability objectives within the present study. It is also important to note that game enjoyment and commitment have been found to increase as a result of including immersive features within a game (DeSmet et al., 2013). For example, immersion in the game context is facilitated by enjoyable narratives that stimulate cognitive and attitudinal change and therefore potentially change health behaviors (Escalas, 2004; Lu et al., 2012; Slater \& Rouner, 2002). However, it has been reported that if too many immersive features are included in the game, then these features can have a counteractive effect on students' performance on the educational task (i.e., parameter for use; (DeSmet et al., 2013)).

An example of an important PT is the use of prompts, as they can trigger certain behaviors-for example, a prompt may be given for the individual to return to the intervention. In "Balance It," students are able to set reminders about the strategies they choose to accomplish their goals at any point in time. As tempting situations are person-specific, it makes sense that tailored prompts are the key to behavior change (Abraham \& Michie, 2008; Bartholomew et al., 2011; Dusseldorp et al., 2013). In line with technological development, triggers on smartphones are even more likely to increase persuasiveness, as they allow for an immediate response. However, certain parameters should be taken into account in order to increase the effectiveness of such prompts (Table 5).

Table 5: Example of the Translation of a Change Objective for Usability into Its Practical Application

\begin{tabular}{|c|c|c|c|c|c|}
\hline $\begin{array}{l}\text { Performance } \\
\text { Objective }\end{array}$ & $\begin{array}{l}\text { Change } \\
\text { Objective }\end{array}$ & Determinant & $\begin{array}{l}\text { Persuasive } \\
\text { Technique }\end{array}$ & Parameter for Use & Application \\
\hline $\begin{array}{l}\text { Students keep } \\
\text { using the inter- } \\
\text { vention }\end{array}$ & $\begin{array}{l}\text { They report } \\
\text { that they have } \\
\text { programmed } \\
\text { prompts them- } \\
\text { selves }\end{array}$ & Trigger & Prompts & $\begin{array}{l}\text { Triggers (signals) } \\
\text { should be } \\
\text { noticed, asso- } \\
\text { ciated with a } \\
\text { behavior, and the } \\
\text { user should be } \\
\text { motivated and } \\
\text { able to perform } \\
\text { the behavior }\end{array}$ & $\begin{array}{l}\text { Triggers are trans- } \\
\text { lated into prompts } \\
\text { sent by a smartphone } \\
\text { application. Prompts } \\
\text { are related to players' } \\
\text { behavioral strategies } \\
\text { and can be actuated } \\
\text { by the individual } \\
\text { player }\end{array}$ \\
\hline
\end{tabular}




\section{IM Step 4: concept intervention program}

In this (final) step of the intervention program development, we integrated all of the previously selected methods (i.e., BCTs and PTs) into the intervention program plan. As this process is very versatile, we will describe the development team, the game elements, the game process, and what resulted from the concept and usability tests that were performed among the target population, respectively.

Development team. An intervention development team was assembled, including behavior change experts, implementation experts, serious game experts, interaction designers, and software engineers. Interaction between team members took place during all stages of concept design, development, and testing. Feedback from members of the user population concerning feasibility, usability, and attractiveness was gathered during all different phases and is described in the paragraph on concept and usability testing.

Game elements. The intervention program ("Balance It") took the form of a serious game design (as they are more likely to increase interactive learning processes and engagement), and it encompasses both educational content (digital game-based learning; (Prensky, 2007) and game elements (Jarvinen, 2007). These game elements can be categorized as systematic elements (i.e., components and the game environment), compound elements (i.e., rule set, game mechanics, theme, interface, and information), and behavioral elements (i.e., players and contexts; see the Appendix for definitions of all game elements).

Systematic game elements. Systematic game elements include game components and the game environment. In "Balance It," the game components are designed as cards that represent "Tetrisshaped" building blocks made out of wood, stone, or metal (i.e., objects that the player is able to manipulate and possess). These cards can be obtained by DI or PA goal accomplishment and/or goal evaluation. By playing these cards, the construction worker piles up the blocks on a small island in the middle of a lake (i.e., the game environment).

Compound game elements. Rule sets, game mechanics, a theme, the interface, and information about the game together form the compound game elements. The game rules of "Balance It" define the goal of the game by producing players' possibilities and constraints (Jarvinen, 2007). This goal could be to 
obtain as many points as possible by building (game mechanic) the highest tower, but the cards that represent the blocks to build this tower can only be obtained by accomplishing a DI- or PA-related task and/or by evaluating one's own behavior. Building this tower and keeping it balanced is used as a metaphor in "Balance It" (theme), referring to the struggle to develop and maintain a healthy lifestyle. The look of the theme is adapted from "cartoony games," as the game experts and user experts suggested that games such as "Angry Birds," "Candy Crush," and "FarmVille" were popular among the target population. The interface of "Balance It" constituted of a smartphone application available on Android $^{\mathrm{TM}}$ (Google, Mountain View, CA) and iOS (Apple, Cupertino, CA) operating systems and connection to the Internet. Finally, a tutorial was embedded in the game to provide information about the outcome, player roles, attributes, and within-game procedures. The specific game procedures are described in the section Game process.

Behavioral game elements. The third category includes the two behavioral game elements players and contexts. "Balance it" targets secondary vocational education students, and the behavioral game element "players" refers to their behavior in terms of health-related goal accomplishment and gameplay, as well as their emotions affected by the level of game difficulty or easiness (Jarvinen, 2007). If the players of "Balance It" experience rewarding positive emotions as a result of gameplay, they may be more motivated to play the game (Jarvinen, 2007). Finally, "Balance It" can be played anywhere and at any time, as long as players are connected to the Internet (context). These and other contextual factors, such as players' personal histories, can possibly affect the game experience. Therefore, it is important to evaluate these behavioral game elements afterward.

Game process. As mentioned previously, "Balance It" will be connected to the present student tracking system of TJL (Bernaards et al., 2011). After students complete a survey on their DI or PA (Figure 2a), they will be referred to the "Balance it" Web site (www.balanceitthegame.nl) (Figure 2b) where they can find information on the health-behavior link, find a forum for social support, download instructions for the "Balance It" application (Figure 2c), and find out how to get a general overview of their progression (Figure $2 \mathrm{~d}$ ). 
Figure 2: Screenshots of (a) "Lifestyle Reality Check", ( $b$ and d) the "Balance It" Website, and (c) the "Balance It" Application
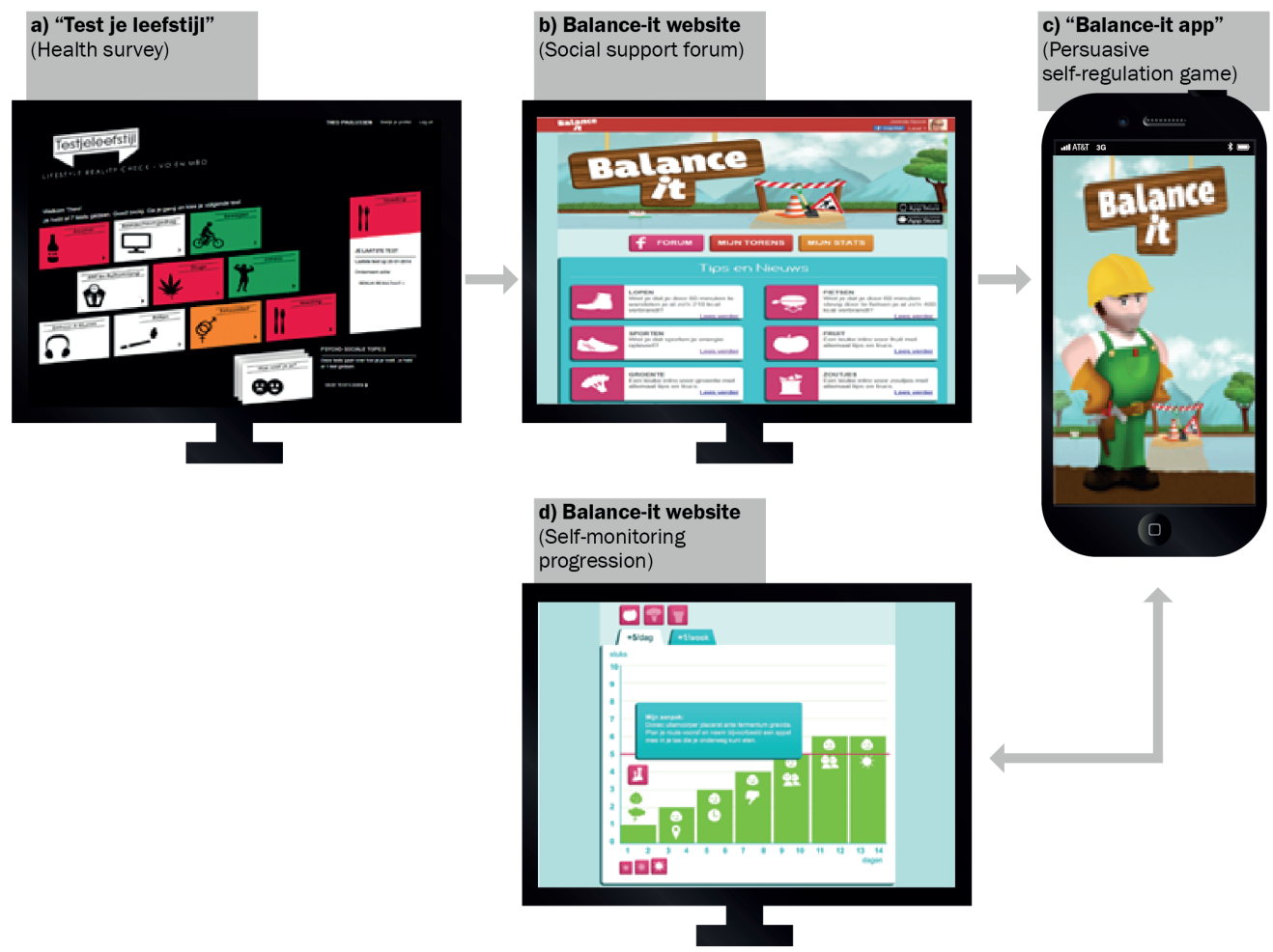

When students first open the "Balance It" application (without any charge), they are given a tutorial, explaining the aim of the game (Figure 3a). Within the tutorial, a construction worker character explains the game to the player (i.e., that he will build the building blocks that the player can earn by accomplishing his or her tasks and by reflecting on his or her own behavior). To tailor gameplay to individual player needs, both individual as well as competitive play options are embedded within the game (Figure 3b). Players are able to invite friends via Facebook to connect with and play against. Each game containing daily tasks (e.g., eat 2 pieces of fruit a day) will be played for 2 consecutive weeks. Games containing weekly tasks (e.g., cycle for 1.5 hours a week) will be played for 6 consecutive weeks. At the start of each game, all players agree on a behavioral contract with themselves or with their opponent, depending on the type of game they decide to play. This behavioral contract is an agreement specifying the specific behavior they will perform themselves and, in case of competitive play, the planned behavior of their opponent. In this way, there is a record of their resolution, also witnessed by the opponent player in case of competitive play, designed to increase cooperation with the program (Abraham \& Michie, 2008) (Figure 3c). 
Figure 3: (a) Tutorial, (b) Individual versus Competitive Game-Play, and (c) Behavioral Contract

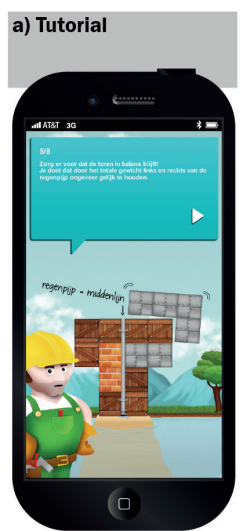

Watch the Balance-it tutorial (optional).

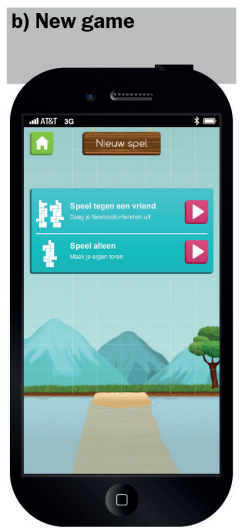

Start an individual or competitive game with a friend.

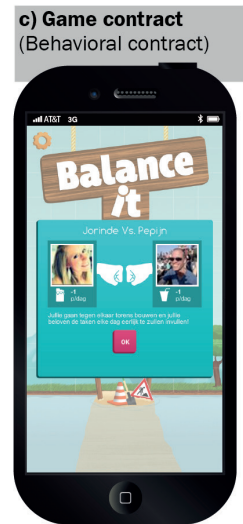

Receive a behavioral contract with self, or opponent player:

Figures 4 (starting a new game) and 5 [daily play of ongoing game(s)] visualize the game process from the moment that a student opens the "Balance It" application. From the start, they can set up a new personal goal, depending on the behavior they would like to work on (e.g., walk more, or eat less candy; Figure 5). They can also go to their game overview to monitor their progression, build blocks, and evaluate their daily or weekly behaviors (according to the goal already set; Figure 5). The self-reevaluation track (i.e., daily evaluation part of the flow chart; Figure 5) encourages players to monitor their own behavior and perceived barriers, create proactive coping plans or, in case of failure, coping plans to reach their goal, and activate prompt notifications to remind them of their coping plans. Examples of coping plans (as implementation intentions) are provided on the Web site linked to "Balance It." Once students have accomplished their goal, they receive two cards that they can use toward building their tower. They receive one card in instances where they have not managed to accomplish their goal but have made an effort to reevaluate their behavior. 
Figure 4: Screenshots of the Game Process when Starting a New Game within the "Balance It" Application and Associated Behavior Change and Persuasive Techniques

\section{STARTING A NEW GAME}

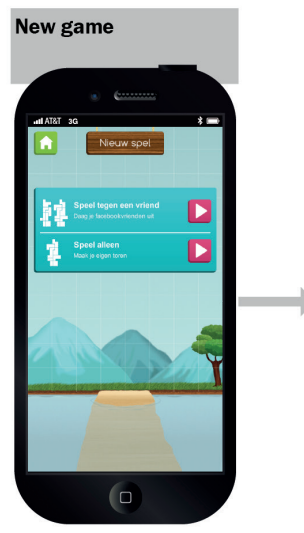

Start an individual or competitive game with a friend.

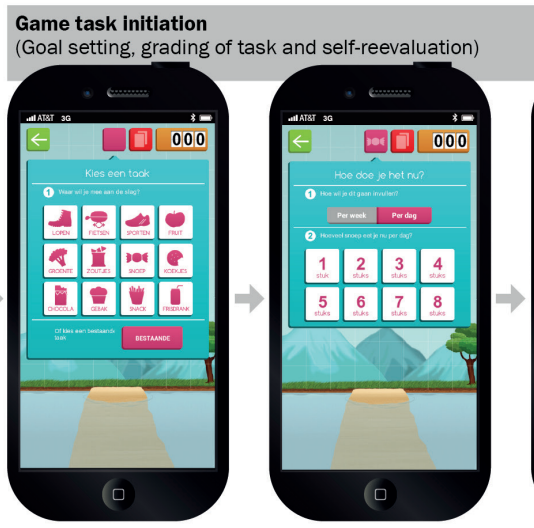

Select a task to work on (e.g. eating candy).
Specify current behavior per week or day (e.g. how much candy do you currently eat per day).

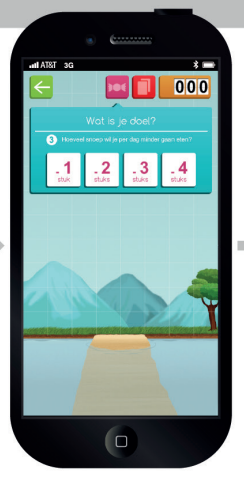

Specify target goal (e.g. how much less candy do you want to eat per day).

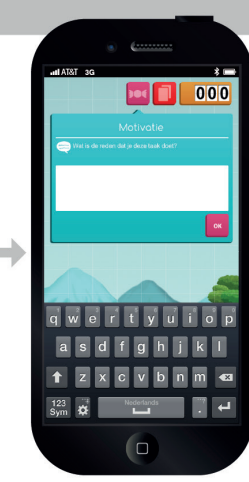

Adda personal motivation to reach goal (optional).

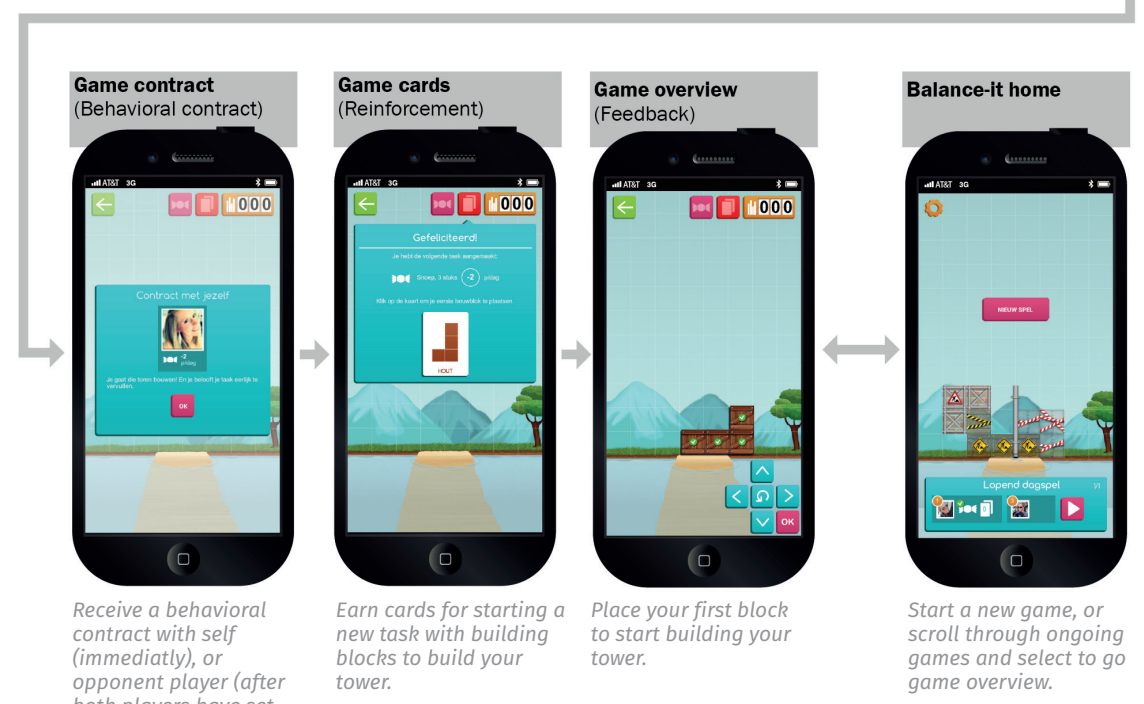
their goal). 
Figure 5: Screenshots of the Game Process for Daily Play Ongoing Game(s) Within the "Balance It" Application and Associated Behavior Change and Persuasive Techniques

\section{DAILY PLAY ONGOING GAME(S)}

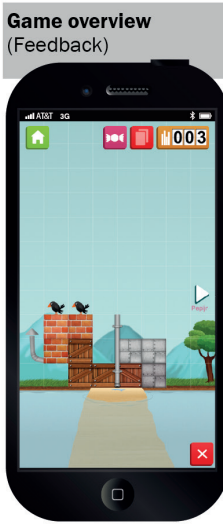

View tower progress and daily reevaluate and monitor your behavior. Build your tower with cards you have earned.

Daily evaluation

(Self-reevaluation and planning of coping responses)

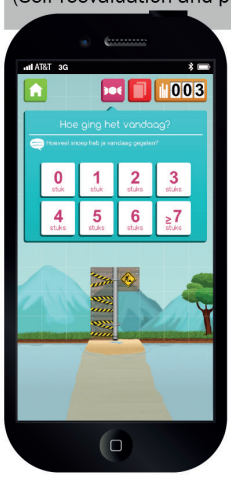

Specify how the day has gone (e.g. how much candy did you eat today?).

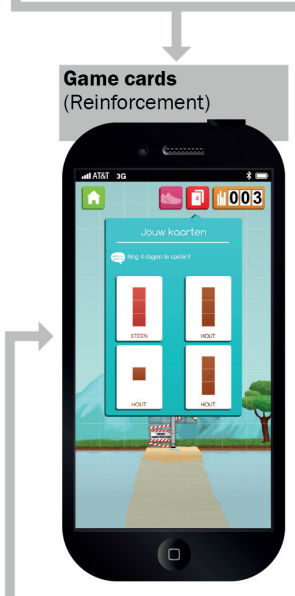

Earn cards for reevaluating your behavior with building blocks to build your tower.

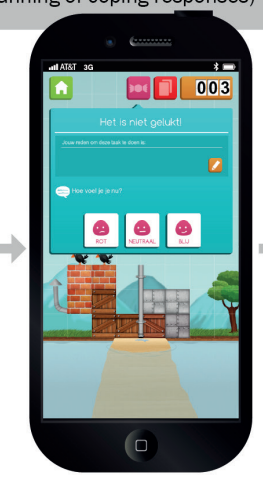

Specify how you feel about this result.

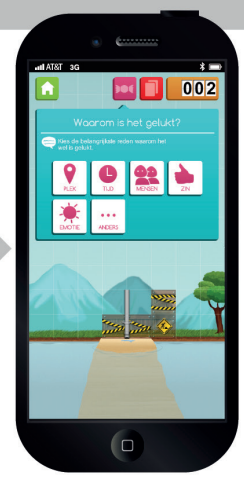

Specify why you did, or did not, reach your goal.

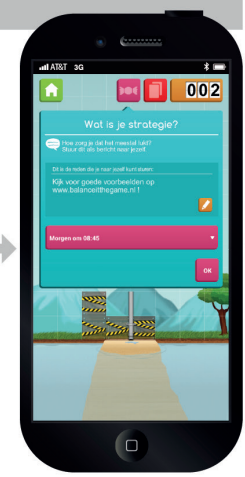

Specify a strategy that will help you reach your goal and set prompts to remind yourself of this strategy (optional).

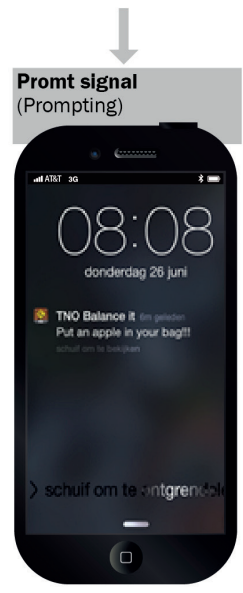

Receive prompt signals with your strategy at set times.

Concept and usability testing. In order to implement the game within an existing student tracking system, the game needed to be proven effective prior to its implementation (DeSmet et al., 2013). To improve the chance of developing an effective intervention program, three concept and usability tests were conducted sequentially and simultaneously to the development of "Balance It" (i.e., tests 
were realized at different points in time but occurred concurrently with the development of "Balance (t"), and students' input was used to optimize the final intervention program, as formative research has been shown to enhance user experience (Crutzen et al., 2013). Results from all three tests were studied, translated, and integrated within the game. In total, 23 secondary vocational education students (74 percent female, 16-18 years of age) from a large city in The Netherlands provided feedback on the program.

The main findings included that the "Balance It" application was perceived as "good-looking," usable, and pleasant, but that a peer support system and information on the health-behavior link were still lacking. In response to this feedback, the "Balance It" Web site was built (www.balanceitthegame.nl) (Figures $2 b-d$ ). Finally, all program objectives and methods were reviewed and were fully integrated into the intervention program.

Only the parameters for the self-monitoring of behavior ("the monitoring must be of the specific behavior; the data must be interpreted and used; the reward must be reinforcing to the individual") are difficult to check within the "Balance it" application, as the data do not provide insight into how exactly students interpret and use the self-monitoring option. In order to tackle this issue, students will be informed about the self-monitoring option within the game prior to gameplay. An overview of the game specifics can be found in Table 6 . 
Table 6: Characteristics of a Self-Regulation Game for Health ("Balance It")

Health topic(s) Improvement of overweight-related behaviors (e.g., dietary intake (DI) and
physical activity (PA))

Targeted age group(s)

Secondary vocational education students

Other targeted group Aged 16-20 years, mainly from low socioeconomic families and minority groups

characteristics

\begin{tabular}{ll}
$\begin{array}{l}\text { Short description of game } \\
\text { idea }\end{array}$ & $\begin{array}{l}\text { Improving students' health-related behaviors by teaching them to regulate their } \\
\text { own behavior (i.e., DI and PA) }\end{array}$ \\
\hline
\end{tabular}

Target player(s) Individual and competitive game players

Guiding knowledge or Self-Regulation Theory, RE-AIM model, FOGG behavioral model

behavior change theory(ies), models, or conceptual

framework(s)

Intended health behavior Dietary intake and physical activity changes

Knowledge element(s) to be Knowledge about health dietary intake and physical activity, benefits, conselearned quences, barrier identification and coping

Behavior change techniques/ Provide information on behavior - health link and consequences, prompt intenmethods tion formation, prompt barrier identification, set graded tasks, provide instructions, prompt specific goal setting, prompt self-monitoring of behavior, provide feedback on behavior, provide contingent rewards, agree on behavioral contract, use follow-up prompts, plan social support, relapse prevent/planning coping responses, implementation intentions, tailoring

Clinical or parental support No

needed?

Data shared with parent or No

clinician

Type of game: Educational

Story

Synopsis (including story arc) The game challenge is to build the highest tower and to keep it in balance, which is challenging as building blocks are shaped as 'Tetris blocks' and are difficult to pile up. Blocks can only be obtained after self-evaluation of the targeted behavior and goal accomplishment

How the story relates to targeted behavior change Building a tower and keeping it balanced is a representation of working on your own health and keep it in balance

Game components

Player's game goal/ Build the highest tower and keep it balanced

objective(s)

Rules

Cards can be obtained by self-reevaluation and goal accomplishment. These cards represent building blocks ('tiles') that can be placed upon their tower by the construction worker. They receive action points for every 'row' of the tower, so the higher the tower is, the more points they will get. Players can also obtain points if they have 4 blocks from the same material in a row

Game mechanic(s) Building, contracting, information-seeking, placing, storytelling, upgrading/ downgrading, prompting 
Procedures to generalize or transfer what has been learned in the game to outside the game
Set a goal, perform the targeted behavior in daily life, evaluate behavior within the game and receive feedback on their 'real time' behavior

Virtual environment

Setting (describe)

Players can start building their towers on a small island in the middle of a lake, surrounded by mountains. As they get higher, the surroundings transform into the universe

Avatar

Characteristics

Construction worker

Abilities

The construction worker builds their tower if they manage to get the cards that tell him which blocks he should use (these blocks have different shapes and are made from different materials)

Game platform(s) needed to Android or iOS (specifically for smartphones)

play the game

Sensors used: No

Estimated play time 2-4 weeks per game

\section{IM Step 5: adoption and implementation plan}

Within this step, we planned strategies that would facilitate the adoption and implementation of the serious self-regulation game intervention as developed in Step 4. It should be noted that considerations for program implementation had already been taken into account during the needs assessment but were revised and elaborated further into clear implementation objectives in IM Step 5. These implementation objectives focus on the adoption, implementation, and sustainability of the game intervention. More specifically, these implementation objectives refer to the tasks of the implementers: the "Lifestyle Reality Check" Foundation and The Netherlands Association of Vocational Education and Training Colleges (in Dutch, MBO Raad). These two implementation agents decided (1) to adopt the program, (2) to implement the program within their existing student tracking system, and (3) to keep the program available to their students to enable continued program usage (cf. (Rogers, 1995)).

With the aim of increasing program usage and achieving intervention effectiveness (DeSmet et al., 2013), we developed an implementation plan in consultation with the "Lifestyle Reality Check" Foundation and the Netherlands Associations of Vocational Education and Training Colleges. This plan referred to the establishment of a link between the current system of TJL and "Balance It" and tackled how survey and process data should be merged (i.e., data from TJL and "Balance It," respectively). 
Once this technical link is established, students will be able to use the intervention independently, without further assistance from third parties. In order to establish this link, the development team had to provide the necessary information about the parameters for use to the engineers behind these two systems.

\section{IM Step 6: evaluation plan}

In this final step of IM we developed a plan to evaluate intervention effectiveness. ${ }^{21}$ This plan includes an evaluation of effectiveness to determine whether "Balance It" successfully changes DI and PA behaviors among secondary vocational education students in The Netherlands. Differences in DI and PA will be measured by means of a pre-/posttest randomized controlled trial, including one intervention group and one waiting list control group. Both groups will be asked to fill out a questionnaire at baseline and during posttest at 4 weeks of follow-up. This questionnaire will assess validated frequency and quantity measures concerning the primary outcomes of dietary behaviors (intake of fruits, vegetables, snacks, and soft drinks) and PA (moderate and vigorous). Secondary outcome measures concerning determinants of DI and PA will also be included (e.g., awareness, intention, attitudes, descriptive and subjective norms, social support, self-efficacy, barrier identification, action planning, coping planning, and self-regulation skills). With an additional process evaluation we aim to determine which mechanisms are effective in improving a healthy diet, increasing PA levels, and ensuring implementation success. With this process evaluation we also aim to collect data on the feasibility and usability of "Balance It." The study protocol of "Balance It" has already been approved by the Ethics Committee of Psychology, Maastricht University, Maastricht, The Netherlands.

\section{Discussion}

The present article describes the design rationale behind a serious self-regulation game intervention called "Balance It." During the development of this intervention we have tried to tackle two important challenges related to intervention effectiveness: accurate application of BCTs and actual intervention use. On the one hand, there are e-health intervention studies that have adequately formulated their objectives, selected their behavior change methods, and translated these methods into online program design, but these studies often report low intervention compliance and high dropout rates (Crutzen et al., 2013). On the other hand, recently developed serious games have shown great potential to be immersive by adopting multiple gamification principles but fall short in applying effective behavior change methods (Crutzen et al., 2013). In order to circumvent these problems, our aim was 
to integrate behavior change methods into serious game design during the systematic development of a serious self-regulation game intervention. In this way, we hope to increase overall effectiveness and intervention use.

A serious game design to change health behavior and to improve intervention usability may have great potential to target low health literacy youth. As suggested by Kreps and Neuhauser, effective e-health interventions are designed to match the unique characteristics, interests, and cultural orientations of the target population (Kreps \& Neuhauser, 2010). The present intervention was designed to tackle this challenge, taking into account the cognitive and social skills and ability of secondary vocational education students, to initiate and maintain healthy behavior. Therefore, multiple concept and usability tests were conducted in which the target population provided feedback on the design and usability of the game "Balance It," while the parameters for BCTs and PTs were taken into account. With a team of behavior change experts, implementation experts, serious game experts, interaction designers, and software engineers, we have sought to develop an effective serious self-regulation game intervention, which will be evaluated soon (i.e., summer 2015).

It should be noted that this integration process of behavior change methods into serious game design can result in incompatibility issues within the design process. In the development of "Balance It," a trade-off had to be made between the choice of behavior change methods and gamification elements. For instance, whereas behavior change experts stressed the importance of a social support system for the students, interaction design experts were concerned with the game process if multiple layers would be added to the game and preferred to focus on game simplicity. As a result, an additional but separate Web site (www.balanceitthegame.nl) was built to stimulate social support and the exchange of game player (coping) strategies regarding health behavior goals. A clear link to the Web site is provided within both the tutorial and the game settings menu. So to create the best of both worlds, trade-offs had to be made.

In line with these trade-offs, we conceptually altered the IM framework (see Figure 1) to emphasize the equal importance of both behavior change processes and intervention use. As suggested by IM, intervention implementation should be anticipated throughout the planning process, even though it is conceptually defined as IM Step 5. However, in practice, intervention studies do not explicitly show how the two are interrelated. E-health intervention implementation is generally described in 
IM Step 5 and focuses on the accessibility and availability of the intervention based on evidence obtained from pilot studies. Even though we advocate the use of pilot studies to identify preferences and needs of the target population regarding the intervention content and design, more needs to be done to enhance intervention use. With the alterations applied to the IM framework, we emphasized the importance of these usability determinants and how we used PTs to facilitate these changes in terms of intervention use. By describing the complete process systematically, we hope to have provided more transparency with regard to intervention development, and serious game intervention development in particular (Baranowski, 2014).

\section{Acknowledgments}

We would like to thank Ijsfontein for the development of "Balance It." The study was funded by TNO, The Netherlands Organisation for Applied Scientific Research.

\section{Author Disclosure Statement}

No competing financial interests exist.

\section{Ontology}

Intervention mapping: A protocol to systematically plan a theory- and evidence-based intervention in six steps: (1) a needs assessment, (2) matrices of change objectives, (3) methods and applications, (4) an intervention program, (5) program adoption and implementation plan, and (6) an evaluation plan (Bartholomew et al., 2011).

- Needs assessment: Identifying what, if anything, needs to be changed and for whom (Bartholomew et al., 2011).

- Performance objectives: Specifying the performance expected from someone affected by the intervention (Bartholomew et al., 2011).

- Determinants: Factors that have been found to be associated with the performance of the behavior of the at-risk population (Bartholomew et al., 2011).

- Change objectives: The determinants of the (sub-)behaviors targeted at identifying who and what will change as a result of the intervention (Bartholomew et al., 2011).

- Behavior change techniques or methods: Techniques derived from theory and research to reach a proximal program objective (Bartholomew et al., 2011). 
- Persuasive techniques: Techniques affecting people's behavioral determinants (Fogg, 2009).

- Intervention program: An organized program in which methods and the practical applications of these methods are integrated (Bartholomew et al., 2011).

- Adoption and implementation plan: A plan for the adoption, implementation and sustainability of the intervention program in real-life contexts (Bartholomew et al., 2011).

- Evaluation plan: A plan to conduct effect and process evaluations (Bartholomew et al., 2011).

Game elements: Generic element classes that get realized as various instances and forms in particular games, specifying systematic, compound and behavioral game elements (Jarvinen, 2007).

- Systematic game elements: Specify the components and the environment of the game (Jarvinen, 2007).

- Components: Objects that the player is able to manipulate and possess in the course of the game (Jarvinen, 2007).

- Environment: The physical or virtual constraints of the game system, embodying rules that specify the spatial arrangement of a game (Jarvinen, 2007).

- Compound game elements: Elements that create connections between game elements (Jarvinen, 2007).

- Rule set: The game rules producing individual possibilities and constraints for game players defining the goal of the game (Jarvinen, 2007).

- Game mechanics: Player action and performance, best described with verbs (e.g., choosing, building, moving, collecting, etc.) (Jarvinen, 2007).

- Theme: The metaphor for the rule set, contextualizing the rule set and its game elements to other meanings than what the game system as an information system requires (Jarvinen, 2007).

- Interface: The medium through which players produce input to the system (Jarvinen, 2007).

- Information: What the system and the player need to know (Jarvinen, 2007).

- Behavioral game elements: Specify the players and the context of a game (Jarvinen, 2007).

- Players: The human factor in the game: their behavior, mood, relationship with games, and game tastes (Jarvinen, 2007).

- Context: The physical location of the game, the time, players' personal histories, and other informal, external aspects to the game system that possibly affect the game experience (Jarvinen, 2007). 


\section{References}

Abraham, C., \& Michie, S. (2008). A taxonomy of behavior change techniques used in interventions. Health Psychology, 27(3), 379-387.

Adriaanse, M. A., Ridder de, D. T. D., \& Wit de, J. B. F. (2009). Finding the critical cue: Implementation intentions to change one's diet work best when tailored to personally relevant reasons for unhealthy eating. PSPB, 35(1), 60-61-71.

Ajzen, I. (1991). The theory of planned behavior. Organizational Behavior and Human Decision Processes, 50, 179-211.

Baranowski, T. (2014). Description for articles introducing a new game for health. Games for Health Journal: Research, Development, and Clinical Applications, 3(2), 55-56.

Bartholomew, L. K., Parcel, G. S., Kok, G., Gottlieb, N. H., \& Fernández, M. E. (Eds.). (2011). Planning health promotion programs: An intervention mapping approach. San Francisco: Jossey-Bass.

Bernaards, C., \& Buuren van, S. (2012). Rapportage veranderingen in het beweeggedrag van mbo studenten. (No. R10185). Leiden, the Netherlands: TNO Behavioural and Societal Sciences.

Bernaards, C., van Keulen, H., Rijpstra, A., \& Paulussen, T. (2011). Beschrijving van de ontwikkeling van een automatische test je leefstijl rapportage per school. (No. 2011.040) TNO.

Brouwer, W., Oenema, A., Crutzen, R., de Nooijer, J., de Vries, N. K., \& Brug, J. (2009). What makes people decide to visit and use an internet-delivered behavior-change intervention? A qualitative study among adults. Health Education, 109, 460-473.

Chambers, J. A., \& Swanson, V. (2012). Stories of weight management: Factors associated with successful and unsuccessful weight maintenance. Britisch Journal of Health Psychology, 17, 223-243.

Crutzen, R., de Nooijer, J., Brouwer, W., Oenema, A., Brug, J., \& de Vries, N. K. (2008). Internet-delivered interventions aimed at adolescents: A delphi study on dissemination and exposure. Health Education Research, 23(3), 427-439. 
Crutzen, R., de Nooijer, J., Brouwer, W., Oenema, A., Brug, J., \& de Vries, N. K. (2009). A conceptual framework for understanding and improving adolescents' exposure to internet-delivered interventions: An example on sexual health. Health Promotion International, 24, $277-284$.

Crutzen, R., Kohl, L., \& de Vries, N. K. (2013). Kennissynthese online preventie. Maastricht, The Netherlands: Universiteit Maastricht.

DeSmet, A., Bastiaensens, S., van Cleemput, K., Poels, K., Vandebosch, H., \& de Bourdeaudhuij, I. (2013). Importance of game and behavioral change theory in creating effective serious video games for health promotion. Gent.

DeSmet, A., van Ryckeghem, D., Compernolle, S., Baranowski, T., Thompson, D., Crombez, G., ... de Bourdeaudhuij, I. (2014). A meta-analysis of serious digital games for healthy lifestyle promotion. Preventive Medicine, 69, 95-107.

Dusseldorp, E., van Genugten, L., van Buuren, S., Verheijden, M. W., \& van Empelen, P. (2014). Combinations of techniques that effectively change health behavior: Evidence from Meta-CART analysis. Health Psychology, 33(12), 1530-1540.

Escalas, J. E. (2004). Imagine yourself in the product: Mental simulation, narrative transportation, and persuasion. Journal of Advertising, 33, 37-48.

Fogg, B. J. (2009). A behavior model for persuasive design. Proceedings of the 4 th International Conference on Persuasive Technology, ACM.

Fogg, B. J., Grudin, J., Nielsen, J., \& Card, S. (2002). Persuasive technology: Using computers to change what we think and do. Science \& Technology Books.

Glasgow, R. E., Vogt, T. M., \& Boles, S. M. (1999). Evaluating the public health impact of health promotion interventions: The RE-AIM framework. American Journal of Public Health, 89(9), 1322-1327.

Gollwitzer, P. M., \& Sheeran, P. (2006). Implementation intentions and goal achievement: A meta-analysis of effects and processes. Advances in Experimental Social Psychology, 38, 69-119.

Jarvinen, A. (2007). Games without frontiers, theories and methods for game studies and design [PhD Dissertation]. Tampere, Finland: University of Tampere. 
Kremers, S. P. J., de Bruin, G., Visscher, T. L. S., van Mechelen, W., de Vries, N., \& Brug, J. (2006). Environmental influences on energy balance-related behaviors: A dual-process view. International Journal of Behavioral Nutrition and Physical Activity, 3(9).

Kreps, G. L., \& Neuhauser, L. (2010). New directions in eHealth communication: Opportunities and challenges. Patient Education and Counseling, 78, 329-336.

Kumanyika, S. K., Obarzanek, E., Stettler, N., Bell, R., Field, A. E., Fortmann, S. P., . . Hong, Y. (2008). Population-based prevention of obesity. the need for comprehensive promotion of healthful eating, physical activity, and energy balance: A scientific statement from american heart association council on epidemiology and prevention, interdisciplinary committee for prevention (formerly the expert panel on population and prevention science). Circulation, 118, 428-464.

Lu, A. S., Baranowski, T., Thompson, D., \& Buday, R. (2012). Story immersion of videogames for youth health promotion: A review of literature. Games for Health Journal: Research, Development, and Clinical Applications, 1(3), 199-204.

Maes, S., \& Karoly, P. (2005). Self-regulation assessment and intervention in physical health and illness: A review. Applied Psychology, 54, 267-299.

Maffeis, C., \& Tato, L. (2001). Long-term effects of childhood obesity on morbidity and mortality. Hormone Research, 55, 42-45.

Marlatt, G. A., \& Gordon, J. R. (1985). Relapse prevention: Maintenance strategies in the treatment of addictive behaviors. New York: The Guilford Press.

Ministery of Education, Culture, and Science. (2012). Kerncijfers 2007-2011. (No. 9879059101562). Kelpen-Oler: Hub Tonnaer B.V.

Must, A. (2003). Does overweight in childhood have an impact on adult health? Nutrition Reviews, 61, $139-142$.

Polder, J. J., \& Takkern, J. (2002). Kosten van ziekten in nederland - de zorgeuro ontrafeld [cost of illness in the netherlands]. (No. 304). Bilthoven: RIVM.

Prensky, M. (2007). Digital game-based learning. St. Paul, Minnesota: Paragon House. 
Prins, R. G. (2012). Environmental influences on physical activity among adolescents [PhD Dissertation]. Rotterdam: Erasmus University.

Rogers, T. (1995). Diffusion of innovations. New York: Free Press.

Ryan, R. M., \& Deci, E. L. (2000). Intrinsic and extrinsic motivations: Classic definitions and new directions. Contemporary Educational Psychology, 25(1), 54-67.

Schnoll, R., \& Zimmerman, B. J. (2001). Self-regulation training enhances dietary self-efficacy and dietary fiber consumption. Journal of American Diet Association, 101, 1006-1011.

Schönbeck, Y., \& van Buuren, S. (2010). Resultaten vijfde landelijke groeistudie. (factsheet). Leiden: TNO.

Seidell, J. C. (2000). The current epidemic of obesity. In C. (. ). Bouchard (Ed.), Physical activity and obesity (2nd ed., pp. 21-30). Champaign, IL: Human Kinetics.

Slater, M. D., \& Rouner, D. (2002). Entertainment-education and elaboration likelihood: Understanding the processing of narrative persuasion. Commun Theory, 12, 173-191.

Spook, J. E., Paulussen, T., Kok, G., \& van Empelen, P. (2013). Monitoring dietary intake and physical activity electronically: Feasibility, usability, and sensitivity of a mobile-based ecological momentary assessment (mEMA) tool. Journal of Medical Internet Research, 15(9), e214.

Spook, J. E., Paulussen, T., Kok, G., \& van Empelen, P., submitted for publication). Factors determining physical activity of emerging young adults: A mobile-based Ecological Momentary Assessment (mEMA) study.

Spook, J.E., Paulussen, T., Kok, G., van Empelen, P., submitted for publication. The role of motivational and contextual factors on intentional and craving-induced snack consumption: A mobile-based Ecological Momentary Assessment study.

Van Genugten, L., van Empelen, P., Flink, I., \& Oenema, A. (2010). Systematic development of a self-regulation weight-management intervention for overweight adults. BMC Public Health, 10, 649.

Van Genugten, L. (2011). Prevention of weight gain among adults: Development and evaluation of a computer-tailored self-regulation intervention [PhD Dissertation]. Rotterdam: Erasmus University. 
Wang, Y., Liang, H., Tussing, L., Braunschweig, C., Cabellero, B., \& Flay, B. (2007). Obesity and related risk factors among low socio-economic status minority students in chicago. Public Health Nutrition, 10(9), 927-938.

Weinstein, N. D., \& Sandman, P. M. (2002). The precaution adoption process model. In K. Glanz, B. K. Rimer \& F. M. Lewis (Eds.), Health behavior and health education. (pp. 121-145). San Francisco: JosseyBass.

Wing, R. R., Tate, D. F., Gorin, A. A., Raynor, H. A., \& Fava, J. L. (2006). A self-regulation program for maintenance of weight loss. The New England Journal of Medicine, 355, 1563-1571.

World Health Organization [WHO] (1998). Health promotion glossary. (No. WHO/HPR/HEP/98.1). Retrieved from: http://www.who.int/healthpromotion/about/HPR\%20Glossary\%201998.pdf?ua=1

World Health Organization [WHO] (2013). Obesity and overweight. (Fact sheet No. 311).WHO.

Zeidner, M., Boekaerts, M., \& Pintrich, P. R. (2000). Self-regulation, directions and challenges for future research. In M. Boekaerts, P. R. Pintrich \& M. Zeidner (Eds.), Handbook of self-regulation (pp. 749-768). San Diego, CA: Academic Press. 



\section{CHAPTER 6}

Evaluation of "Balance It": A Pilot Study on the Effects of a Serious

Self-Regulation Game Intervention for Overweight-Related Behaviors

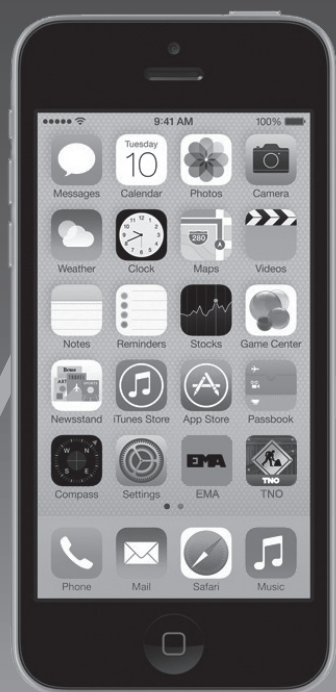




\section{Evaluation of "Balance It": A Pilot Study on the Effects of a Serious Self-Regulation Game Intervention for Overweight-Related Behaviors}

\section{Abstract}

Background: The present study evaluated the effects of "Balance It", a serious self-regulation game intervention targeting dietary intake (DI) and physical activity (PA) designed to prevent overweight among secondary vocational education students.

Objective: To examine the effects of "Balance It" on secondary vocational education students' DI and PA.

Methods: In total, 501 secondary vocational education students participated at baseline ( $N_{\text {intervention }}$ group $=250, N_{\text {control group }}=251$ ) in the present Group-Randomized Trial (GRT). After four weeks, at immediate post-test, 231 students filled in the post-test questionnaire $\left(N_{\text {intervention group }}=105, N_{\text {control group }}=\right.$ 126). The sample had an average age of $17(S D=1.30$, range $15-21), 62 \%$ were female, and $26 \%$ had a non-Dutch background. Body Mass Index (BMI) ranged from 14.4 to $31.1(M=21.1, S D=3.3)$. Behavioral outcomes were dietary intake (DI: fruit and vegetable consumption, snack consumption, and softdrink consumption), and physical activity (PA: moderate + vigorous). Determinants included attitude, self-efficacy (SE), intention, barrier identification, action planning, and action control.

Results: The intervention group showed a significantly stronger decrease in soft-drink consumption $\left(\Delta \mathrm{M}_{\text {Intervention Group }}=-.15, \Delta \mathrm{M}_{\text {Control group }}=-.04 ; P<.05\right)$, and a significant stronger increase in the use of active transport (i.e., walking or cycling as a way of transportation; $\Delta M_{\text {Intervention Group }}=.65, \Delta M_{\text {control group }}=-.12$; $P<.01)$., However, they also reported less positive healthy $\mathrm{DI}$ attitudes $\left(\Delta \mathrm{M}_{\text {Intervention Group }}=-.08, \Delta \mathrm{M}_{\text {control }}\right.$ group $=.02 ; P<.01)$, lower self-efficacy in relation to a healthy $\mathrm{DI}\left(\Delta \mathrm{M}_{\text {Intervention Group }}=-.29, \Delta \mathrm{M}_{\text {control group }}=\right.$ $-.16 ; P<.01)$, and less PA intentions $\left(\Delta M_{\text {Intervention Group }}=-.31, \Delta M_{\text {control group }}=-.19 ; P<.01\right)$. As only $28 \%$ of the intervention group reported actual intervention use, this subset of intervention users $(N=29)$ were compared with non-users $(N=200)$. Compared to non-users, intervention users increased fruit intake $\left(\beta=.40, P=.003, R^{2}\right.$ change $\left.=.04\right)$ and vegetable intake $\left(\beta=.15, P=.02, R^{2}\right.$ change $\left.=.03\right)$, and decreased snack consumption $\left(\beta=-.20, P=.02, \mathrm{R}^{2}\right.$ change $=.03$ ). Intervention users also showed increased positive attitudes towards PA $\left(\beta=.32, P=.01, \mathrm{R}^{2}\right.$ change $\left.=.03\right)$ as well as an increase in PA intentions $(\beta=.41$, $P=.05, \mathrm{R}^{2}$ change $=.02$ ). The "intervention use" group also reported enhanced action control skills for healthy eating $\left(\beta=.65, P=.01, R^{2}\right.$ change $\left.=.04\right)$, unhealthy eating $\left(\beta=.55, P=.02, R^{2}\right.$ change $\left.=.03\right)$, and $\mathrm{PA}\left(\beta=.64, P=.01, \mathrm{R}^{2}\right.$ change $\left.=.04\right)$.

Discussion: As intervention use was still not optimal, future research on the effects of "Balance It" 
should take place once the intervention has already been embedded within an existing student tracking system. In summary, "Balance It" shows promise for the further development and use of serious games designed to target health-related behaviors among youth.

\section{Introduction}

Overweight and obesity are related to various chronic health problems, including diabetes type 2 , cardiovascular disease, cancer, and also psychosocial problems (Eisenberg, Neumark-Sztainer, \& Story, 2003; Formiguera \& Canton, 2004; Roberts, Deleger, Strawbridge, \& Kaplan, 2003; Storch et al., 2007; Stunkard, Faith, \& Allison, 2004; World Health Organization, 2004). In the Netherlands, about $20 \%$ of youth (16-20 years of age) from low socioeconomic (SES) families are overweight or obese (Bernaards \& Buuren van, 2012), and prevalence is even higher among youth with Turkish or Moroccan descent (Shönbeck \& van Buuren van, 2010; Wang, Monteiro, \& Popkin, 2002; Wang et al., 2007). Treatment of overweight remains a challenge, and hence, it is important to target overweight-related behaviors (e.g., dietary intake (DI) and physical activity (PA)) in intervention studies designed to prevent overweight in low SES youth.

Recent advances in technology enable researchers to tailor DI and PA interventions to the needs of the target population. Moreover, it is possible to design a program that is cost effective, that has a wide reach, and that can function as a standalone program (Tate, 2008; van Keulen et al., 2011). Although reviews highlight the potential computer-tailored interventions show in terms of effectively changing and promoting health-related behaviors (Krebs, Prochaska, \& Rossi, 2010; Kroeze, Werkman, \& Brug, 2006; Nguyen, Kornman, \& Baur, 2011), targeting young people, immigrant groups, people with a low, primary or basic vocational education, and people with a weak health motivation can still be challenging (van't Riet, Crutzen, \& de Vries, 2010). To overcome hurdles such as low reach and limited adoption of computer-tailored interventions, several strategies have been recommended, such as increasing the interactivity and visual attractiveness of the program (Crutzen et al., 2008; Crutzen et al., 2011; DeSmet et al., 2014). Serious gaming is a promising method that can be used to stimulate intervention use, as such games are designed to be highly enjoyable, attention-grabbing and intrinsically motivating (Baranowski, Baranowski, \& Cullen, 2003; Baranowski, Buday, Thompson, \& Baranowski, 2008; Graesser, Chipman, Leeming, \& Biedenback, 2009; Prensky, 2007). In previous research (Baranowski et al., 2011), serious gaming interventions (such as "Diab" and "Nano") appeared to increase fruit and vegetable intake. Playing these games, however, did not 
increase water consumption, PA, or body composition. Thompson et al. (2015) indicated that action intentions may be an important component of successful interventions to stimulate youth fruit and vegetable intake (Thompson et al., 2015), which in combination with coping plans may also account for PA (Araújo-Soares, McIntyre, \& Sniehotta, 2009).As such, these studies showed that serious games have great potential to change health-related behaviors. According to DeSmet et al., however, serious games generally fall short in applying effective behavior change methods to change health-related behaviors (DeSmet et al., 2014). DeSmet et al. advocate the use of dual theoretical frameworks, stressing the importance of a theoretical foundation in both behavioral prediction and game theories (DeSmet et al., 2014). To this end, we combined effective behavior change techniques (as applied in computer-tailored interventions) with serious gaming strategies in order to encourage intervention use and target health behavior change simultaneously. As such, we developed a serious self-regulation game intervention called "Balance It".

"Balance it" combines behavior change techniques derived from self-regulation theory (Maes \& Karoly, 2005; Van Genugten, Van Empelen, Flink, \& Oenema, 2010) with serious game elements. It is a serious self-regulation game designed to target DI and PA among secondary vocational education students. "Balance It" was systematically developed by means of Intervention Mapping (IM), a protocol that enables the systematic planning of theory- and evidence-based interventions (Bartholomew, Parcel, Kok, Gottlieb, \& Fernández, 2011). Further elaboration on the design rationale of "Balance It" can be found elsewhere (Spook, Paulussen, Kok, \& Van Empelen, 2015). Our key research objectives in the present pilot study were to: (1) identify the effectiveness of "Balance It" on changes in (determinants) of secondary vocational education students' DI and PA, and (2) evaluate the uptake and usage of the game, and the game elements.

\section{Methods}

\section{Study Design}

A Group-Randomized Trial (GRT) was conducted in 2014/2015 with measurements taken at baseline, immediately post-test (after four weeks of game play), and at a four-week follow-up. Fifteen vocational education schools in the Netherlands were approached to participate in the present study. In total, four schools agreed to participate and were randomly assigned to the intervention or waiting list control group. To counteract contamination effects between participant groups and to increase 
participants' compliance with the study, random allocation to conditions took place at the level of schools (Donner \& Klar, 2000). All procedures were approved by The Research Ethics Board of the School of Psychology \& Neuroscience (Maastricht University).

\section{Participants}

In total, 501 secondary vocational education students participated at baseline $\left(N_{\text {intervention group }}=250\right.$, $\left.N^{\text {control group }}=251\right)$. At post-test, 231 students participated for a second time and were included in the present study $\left(N_{\text {intervention group }}=105, N_{\text {control group }}=126\right.$; see Figure 1$)$. The final sample was $62 \%$ female $(N=311)$ and $26 \%$ non-Dutch $(N=130)$, with an average age of $17(S D=1.30$, range 15-21). Based on self-reported height and weight, Body Mass Index (BMI) ranged from 14.4 to $31.1(M=22.0, S D=3.2)$. Students who had no smartphone operating on iOS or Android were exempt from participation, along with students younger than 16 or older than 21 years old. Students under 16 were exempted from participation as within the Netherlands, individuals are allowed to provide informed consent from the age of 16 . Outlier analyses indicated participants above 21 as outliers, and as such we specifically targeted students between the age of 16-21. All other students were eligible for participation. 
Figure 1: Flow Diagram of the Enrollment and Selection of Study Participants

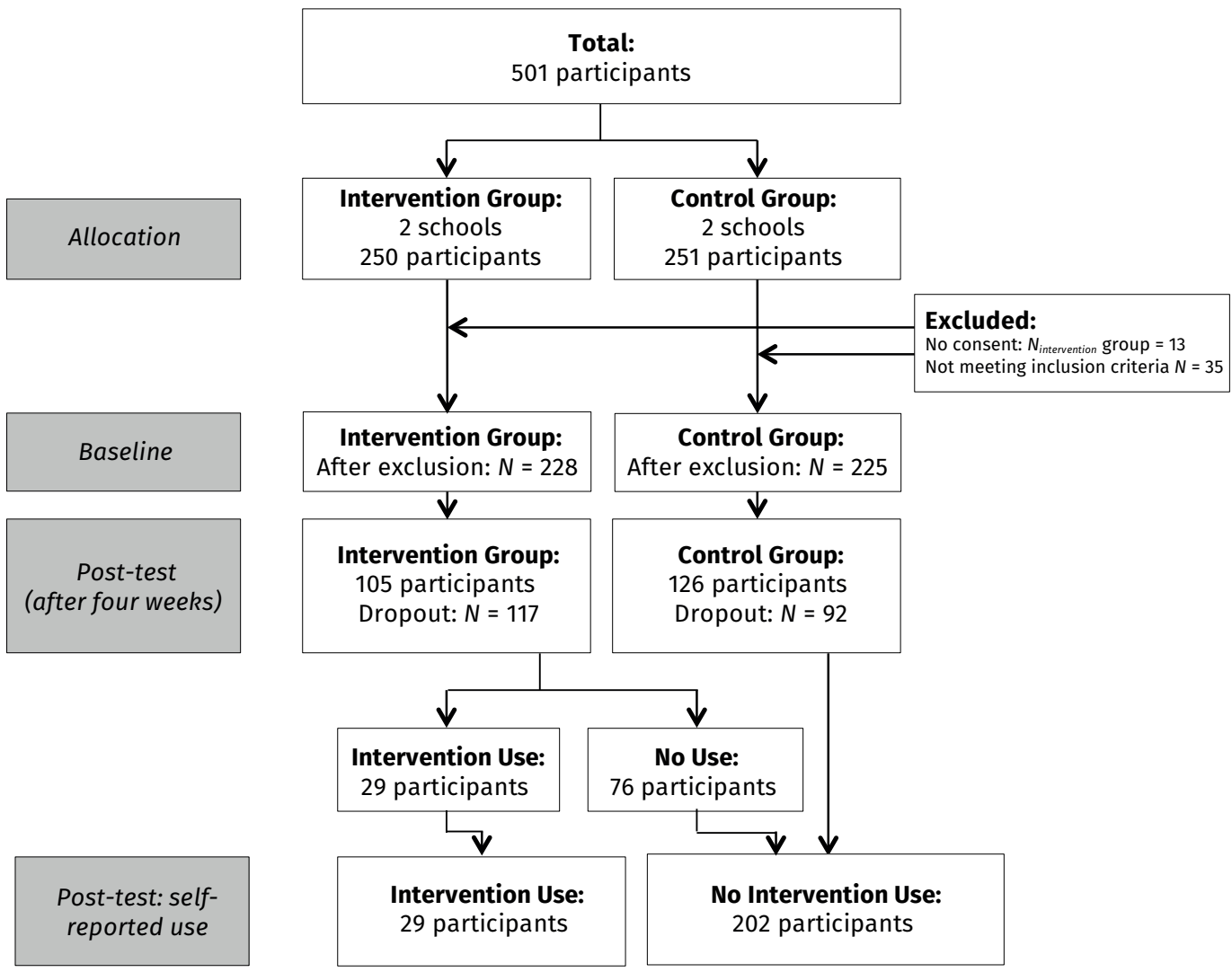

\section{Procedures}

One week prior to the study, participants received passive consent forms addressed to their parents or caregivers. At baseline, a research assistant went to the schools to introduce the study and to collect the survey data. In total, 238 students gave their consent to participate in the present study. At baseline, participants filled out an online baseline questionnaire regarding their average DI and PA, social cognitive factors (i.e., attitude, SE, intention), perceived barriers, self-regulation skills, action planning, and action control. After they finished the questionnaire, participants received a link to the "Balance It" website and further instructions about downloading the "Balance It" app from the research assistant. All students received a post-test questionnaire four weeks after the baseline measure was taken. Participatory incentives of $€ 20$ vouchers were randomly distributed among participants. The chance of winning a voucher increased with the number of measures completed (one measure 1:8, two measures 2:8). 


\section{"Balance It"}

"Balance It" was designed as a tailored, interactive multimedia game in which each game could be played either individually or competitively with others, at any time and place desired. It was designed as an educational, strategic game that could be played on a daily basis for four continuing weeks or on a weekly basis for six continuing weeks. Within each game, players set graded tasks themselves (e.g., to eat two pieces of fruit per day), which were selected from a multiple choice format (see Figure 2 and Spook et al., 2015). They monitored and evaluated these goals on a daily or weekly basis, depending on the type of game they chose to play. Each day or week, players were prompted with their goals and reminded to return to the game. Visual feedback on (self-reported) goal attainment was provided for each goal, and players were prompted to reflect on their condition and on the perceived barriers or facilitators of goal accomplishment. Finally, participants were encouraged to formulate implementation intentions. In turn, these implementation intentions, or strategies, could be set as reminder prompts at any specific time point the player preferred. Information about formulating implementation intentions was provided on the "Balance It" website. The website also provided a general overview of the participant's progress and a peer-support system (i.e., the "Balance It" forum). Reinforcement was given in the form of obtainable "Tetris-shaped" building blocks and the allocation of "super powers" after goal accomplishment and self-evaluation of the targeted behavior. With these building blocks players were encouraged to build a tower and to keep the tower in balance (see Figure 2, and for a full description of the game design and content see Spook et al., 2015). 
Figure 2: Screenshots of task initiation in the "Balance It" app

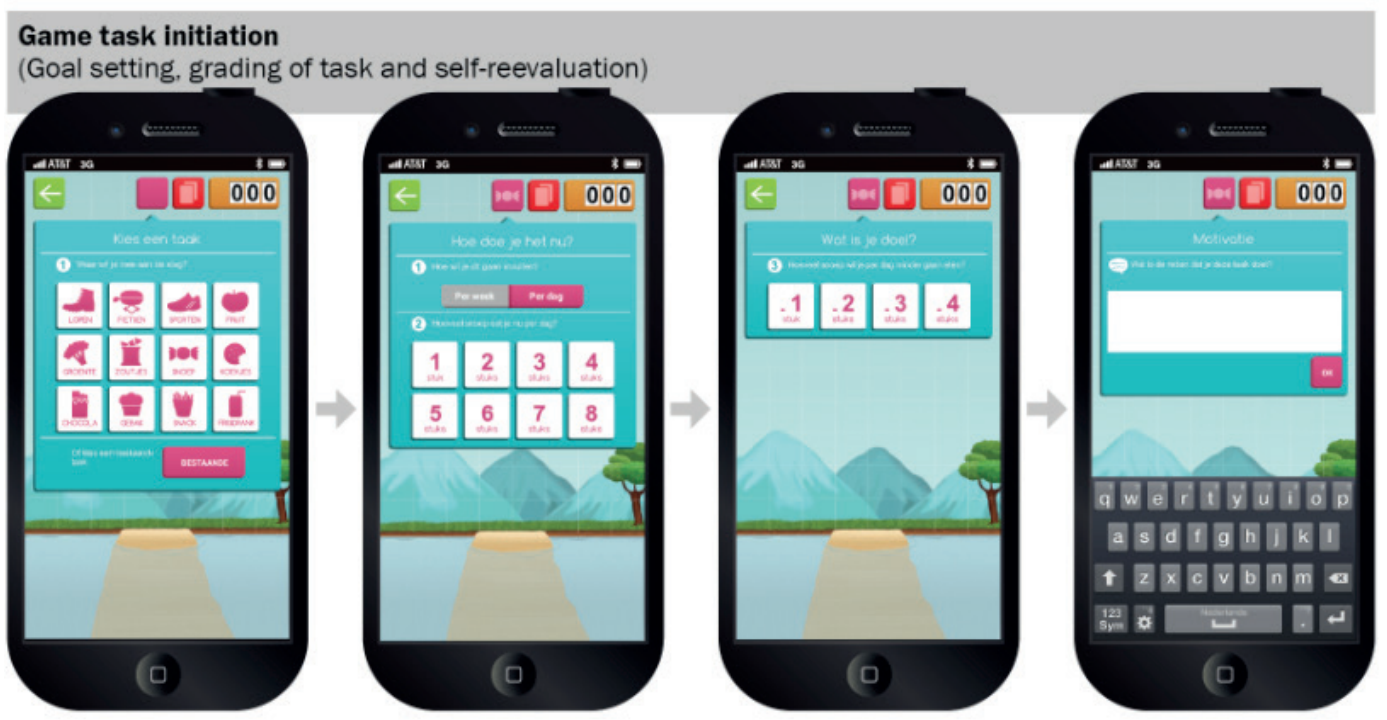

\section{Waiting List Control Group}

At baseline, the control group was instructed to fill in the baseline questionnaire and informed that the researcher would return in four weeks for a post-test measure. In between measures, no interventions were offered by the researchers. Immediately following post-test, students were provided with information about "Balance It" and given the opportunity to play.

\section{Behavioral Outcome Measures}

Dietary intake. The assessment of dietary intake was derived from a validated food frequency questionnaire (Assema van, Brug, Ronda, \& Steenhuis, 2001). Questions were related to the participant's average daily fruit and vegetable intake, snack consumption, and soft-drink consumption. Answers were given on an 8-point scale on which participants could record the number of days they consumed specific foods, ranging from 0 ((almost) never) to 7 (every day). In addition, the quantity of their dietary intake was assessed. Response categories ranged from 1 ( $1 / 2$ portion or piece a day) to 7 (3 or more portions or pieces a day). Based on these scores, the average intake per day was calculated.

Physical activity. PA measures were derived from the OBiN questionnaire (validated by (Douwes \& Hildebrandt, 2000). Questions were related to the participant's average moderate PA (walking and 
cycling) and vigorous PA (exercise). For example, moderate PA was operationalized as "During the last week, how many days did you carry out 30 minutes of moderate PA?" Answers were given on an 8-point scale on which participants could rate the number of days they were moderately or vigorously active, ranging from $0=(($ almost $)$ never $)$ to 7 = (every day $)$.

\section{Determinants of Dietary Intake}

In addition to the behavioral outcomes, social cognitive factors were measured for healthy dietary intake (fruit and vegetable intake) and unhealthy dietary intake (snacks, sweets and soft-drink consumption). All measures of determinants were preceded by a stem, followed by the behavioral outcome measures as subcategories.

Attitude -DI was assessed by three items using semantic differential response scales, e.g., "I think that eating two pieces of fruit a day is..." ( $1=$ very bad to $5=$ very good; $1=$ very unpleasant to $5=$ very pleasant; 1 = very unhealthy to 5 = very healthy; cf. Van der Horst, Oenema, Van de Looij-Jansen, \& Brug, 2008; Van Genugten, Van Empelen, \& Oenema, 2014). Cronbach's alpha for the healthy DI attitude items was .87 and Cronbach's alpha for the unhealthy DI attitude items was .87 .

Self-Efficacy (SE) - DI was assessed by one item preceded by a question stem: "If I want to, I am capable of..." Items were derived from Van der Horst. et al. (2008) and from Van Genugten et al. (2014). Response options ranged from 1 (definitely not) to 5 (definitely). Cronbach's alpha for the healthy DI SE items was .82. Cronbach's alpha for the unhealthy DI SE items was .88.

Intention -DI was assessed with one item preceded by the stem: "I planned to..." (cf. van der Horst et al., 2008; Van Genugten et al., 2014). Response options ranged from 1 (definitely not) to 5 (definitely). Cronbach's alpha for the healthy DI Intention items was .82. Cronbach's alpha for the unhealthy DI Intention items was 93 .

Barrier Identification - healthy DI was assessed separately from unhealthy DI, as different barriers influence fruit and vegetable intake and unhealthy DI. Healthy DI was assessed with five items using five-point Likert scales: "I am capable of eating sufficient fruit and vegetables, also when I am..." Response options ranged from 1 (definitely not) to 5 (definitely). Subcategories referred to: when I am alone, during the weekend, when I am in a hurry, when I experience difficulties preparing fruits and 
vegetables, and when there is a lack of choice. Items were derived from previous measures (Spook, Paulussen, Kok, \& Van Empelen, 2013; Spook, Paulussen, Kok, \& Van Empelen, submitted for publication; Spook, Paulussen, Kok, \& Van Empelen, submitted for publication). Cronbach's alpha for the unhealthy DI Barrier Identification items was .86.

Barrier Identification - unhealthy DI was assessed with 13 items using five-point Likert scales: "I am capable of eating a limited amount of unhealthy snacks, also when I am..." Response options ranged from 1 (definitely not) to 5 (definitely). Subcategories referred to physical settings (e.g., when I am at home), sedentary activities (e.g., when I am watching TV), social settings (e.g., when I am at a party), and mood (e.g., when I am sad). Items were derived from previous measures (Spook et al., 2013; Spook et al., submitted for publication; Spook et al., submitted for publication). Cronbach's alpha of the unhealthy DI Barrier Identification items was .96.

Action Planning -DI was assessed by four items, e.g., "I have a clear plan for when I..." Response options ranged from 1 (definitely not) to 5 (definitely).Subcategories referred to when, where, how, and how often participants planned to eat more healthy or less unhealthy foods (cf. (Van der Horst et al., 2008; Van Genugten et al., 2014). Cronbach's alpha for the healthy DI Action Planning items was .97. Cronbach's alpha for the unhealthy DI Action Planning items was .96.

Action Control -DI was measured with four items using five-point Likert scales, e.g., "During the last month, I have constantly monitored my..." Response options ranged from 1 (definitely not) to 5 (definitely). Subcategories referred to self-monitoring of fruit and vegetable consumption, awareness of fruit and vegetable standards, self-regulatory effort to eat more healthy and less unhealthy foods, and self-regulatory effort to conform to norm behavior (e.g., eat two pieces of fruits a day) (cf. (Sniehotta, Nagy, Scholz, \& Schwarzer, 2006). Cronbach's alpha for the healthy DI Action Planning items was .94. Cronbach's alpha for the healthy DI Action Planning items was .94.

\section{Determinants of PA Physical Activity}

Social cognitive factors were also measured for moderate physical activity (PA; i.e., walking and cycling), and vigorous PA (i.e., exercising). All measures of PA determinants were preceded by a stem, followed by the behavioral outcome measures subcategories. 
Attitude -PA was assessed by three items using semantic differential response scales, e.g., “I think that exercising is..." ( $1=$ very bad to $5=$ very good; $1=$ very unpleasant to $5=$ very pleasant; $1=$ very unhealthy to 5 = very healthy) (cf. Van der Horst et al., 2008; Van Genugten et al., 2014). Cronbach's alpha for the PA items was 79.

Self-Efficacy (SE) - PA was assessed by one item preceded by a question stem: "If I want to, I am capable of..." Items were derived from Van der Horst. et al. (2008) and from van Genugten et al. (2014) (Van der Horst et al., 2008; Van Genugten et al., 2014). Response options ranged from 1 (definitely not) to 5 (definitely). Cronbach's alpha for the PA SE items was .75.

Intention -PA was assessed with one item preceded by the stem: "I planned to..." (cf. van der Horst et al., 2008; Van Genugten et al., 2014). Response options ranged from 1 (definitely not) to 5 (definitely). Cronbach's alpha for the PA Intention items was.73.

Barrier Identification - PA was assessed with seven items using five-point Likert scales, e.g., “I am capable of being more physically active, also when I am..." Response options ranged from 1 (definitely not) to 5 (definitely). Subcategories referred to: when I am busy, when I am stressed, if I failed last time, when I am tired, when it's raining, if I don't have the time, and if I don't get social support (cf Spook et al., 2013; Spook et al., submitted for publication; Spook et al., submitted for publication). Cronbach's alpha for the PA Barrier Identification items was .93.

Action Planning -PA was assessed by four items using five-point Likert scales, e.g., "I have a clear plan for when I..." Response options ranged from 1 (definitely not) to 5 (definitely). Subcategories referred to: when, where, how and how often participants planned to be more physically active (cf. van der Horst et al., 2008; Van Genugten et al., 2014). Cronbach's alpha for the PA Action Planning items was .96.

Action Control -PA was measured with four items using five-point Likert scales, e.g., "During the last month, I have constantly monitored my..." Response options ranged from 1 (definitely not) to 5 (definitely). Subcategories referred to: self-monitoring of PA, awareness of PA standards, self-regulatory effort to be more physically active, and self-regulatory effort to conform to norm behavior (e.g., to be moderately active) (cf. Sniehotta et al., 2006). Cronbach's alpha for the PA Action Planning items was .94. 


\section{Demographics}

Items regarding gender, age, BMI, educational level, cultural background, accommodation and living situation were included at the beginning of the baseline measure. Ethnicity was defined according to the procedures of Statistics Netherlands; individuals were considered to have a Dutch background if both parents were born in the Netherlands. If one of the parents was born outside the Netherlands, the student was considered to have a non-Dutch background (Statistics Netherlands, 2000).

\section{Self-reported process measures}

To evaluate subjective experience of using the "Balance It" app and/or website, 19 items regarding the "Balance It" app in general were preceded by a stem: "What did you think of...". Response options ranged 1 (very bad) to 5 (very good) (cf. attitude measures; Van der Horst et al., 2008; Van Genugten et al., 2014). Cronbach's alpha for the attitude towards the "Balance It" app in general was .98 The following thirteen items were preceded by the same stem and referred to the specific game elements included (e.g., "What did you think of the theme of "Balance It"?). Response options ranged from 1 (very stupid) to 5 (very funny). (cf. attitude measures (Van der Horst et al., 2008; Van Genugten et al., 2014). Cronbach's alpha for the attitude towards game elements was .98.

\section{Statistical analyses}

Descriptive statistics were used to characterize both study groups at baseline (i.e., gender, age, educational level, ethnicity, and BMI). Chi-square tests and t-tests were conducted to evaluate whether participant characteristics were related to drop-out during the study. Repeated measures analyses were performed, using linear regression models to study change scores between pre- and post-test, and differences between the intervention and control groups for both behavioral and determinant measures. Second, similar analyses were conducted to study the effects of self-reported intervention use. A p-value of 0.05 or lower was considered to be statistically significant. All analyses were conducted with SPSS 20.0. 


\section{Results}

\section{Participants \& Dropout Analysis}

In total, 501 students were invited to participate in the present GRT (i.e., $N_{\text {Intervention Group }}=250, N_{\text {control }}$ Group $=251$; Figure 1). Of all students invited, 488 participated (total N, 97\%). Six students were excluded because they were under the age of 15 and 29 students were excluded because they were over 21 years of age After exclusion, 228 participants in the intervention group and 225 in the control group remained at baseline. After four weeks, 117 participants dropped out from the intervention group, and 92 participants dropped out from the control group. Logistic regression analyses revealed that participants who dropped out were significantly older $(M=18$ years, $S D=1.53)$ than non-dropouts $(M=17$ years, $S D=1.26 ; O R=0.8,95 \% \mathrm{Cl} .71-.93)$. Chi-square tests also showed that students with a non-Dutch background were more likely to dropout $(N=119,57 \%)$ than students with a Dutch background $(N=86,41 \% ; \mathrm{OR}=2.0,95 \% \mathrm{Cl} 1.31-2.92 ; 2 \%$ of student backgrounds was unknown $[N=$ 4]). We did not find any significant differences in terms of gender, level of education, year of education or BMI. The BMI distribution of the sample is comparable with previous research on secondary vocational education students' health and weight (Rijpstra \& Bernaards, 2011). At the last count, 105 participants were included in the intervention group and 126 participants were included in the control group.

Of all participants in the intervention group $(N=105), 28 \%$ reported actual intervention use $(N=29)$. Compared to the non-users (i.e., $72 \%$ of the "intervention group" + the control group; total $N=200$ ), (self-reported) intervention users were less likely to participate in the Vocational Education Sector (VES) "Care and Well-being" ( $P \leq .001)$ and more likely to participate in the VES "Economics" $(P \leq .001)$. Participants in the "intervention use" group were all in their first year, compared to $80 \%$ within the "non-use" group (i.e., $N=160 ; \mathrm{P}=.01$ ). The two groups did not significantly differ in age, gender, ethnicity, BMI, VES “Social”, VES “Technique”, or level of education.

\section{Baseline differences}

Table 1 presents the demographic background of Balance It participants at baseline $(N=231)$. Compared to the control group, participants in the intervention group were younger. They were also more likely to participate in the VES Economics, and less likely to participate in Care and wellbeing, Social Work, and Economy. Finally, they were more likely to attend the first year of secondary 
vocational education. We therefore controlled for these differences in the following analyses. We also controlled for baseline differences between the intervention group and the control group in case they differed on behavioral outcome and determinant measures. As such, the intervention group at baseline was more likely to use active transport as compared to the control group ( $P=.04)$. We therefore controlled for the use of active transport at baseline in further analyses concerning active transport.

Table 1: Demographic Background of "Balance It" Participants at Baseline

\begin{tabular}{|c|c|c|c|c|}
\hline \multirow[t]{3}{*}{ Variables } & & \multicolumn{2}{|c|}{ Groups (N = 231) } & \multirow{3}{*}{$\begin{array}{l}\text { Test for difference } \\
\text { (t-test for age, } \\
\text { chi-square for other } \\
\text { variables) }\end{array}$} \\
\hline & & \multirow{2}{*}{$\begin{array}{c}\text { Intervention } \\
\text { group } \\
(N=105)\end{array}$} & \multirow{2}{*}{$\begin{array}{l}\text { Control } \\
\text { group } \\
(N=126)\end{array}$} & \\
\hline & & & & \\
\hline \multicolumn{5}{|c|}{ Demographic variables } \\
\hline Age & Mean (SD) & $16.96(1.10)$ & $17.52(1.36)$ & $-3.34^{*}$ \\
\hline Gender & $\%$ male $(N)$ & $38 \%(39)$ & $37 \%(47)$ & .01 \\
\hline Ethnicity & $\% \operatorname{Dutch}(N)$ & $75 \%(77)$ & $73 \%(92)$ & .09 \\
\hline Track & $\%$ Vocational Training $(N)$ & $97 \%(97)$ & $97 \%(120)$ & .01 \\
\hline \multirow[t]{4}{*}{ VES } & $\%$ Care and well-being $(N)$ & $26 \%(25)$ & $97 \%(115)$ & $108.39 * *$ \\
\hline & $\%$ Economics $(N)$ & $73 \%(71)$ & $0 \%(0)$ & $128.90 * *$ \\
\hline & $\%$ Technique $(N)$ & $1 \%(1)$ & $2 \%(2)$ & .16 \\
\hline & \% Social work (N) & $0 \%(0)$ & $1 \%(1)$ & .81 \\
\hline \multirow[t]{2}{*}{ Educational level } & $\%$ Level $3(N)$ & $5 \%(5)$ & $7 \%(9)$ & .48 \\
\hline & $\%$ Level $4(N)$ & $95 \%(95)$ & $93 \%(115)$ & .48 \\
\hline \multirow[t]{2}{*}{ Year of education } & $\%$ Year $1(N)$ & $100 \%(100)$ & $68 \%(84)$ & $39.27^{\star *}$ \\
\hline & $\%$ Year $2(N)$ & (0) & $34 \%(40)$ & \\
\hline \multirow[t]{4}{*}{ Living situation } & $\%$ Both parents $(N)$ & $77 \%(79)$ & $79 \%(100)$ & 5.30 \\
\hline & $\%$ One parent $(N)$ & $18 \%(19)$ & $13 \%(16)$ & \\
\hline & $\%$ Alone $(N)$ & $2 \%(2)$ & $2 \%(2)$ & \\
\hline & $\%$ Other $(N)$ & $3 \%(3)$ & $6 \%(8)$ & \\
\hline \multirow[t]{4}{*}{ BMI categories } & \% Underweight $(\mathrm{BMI}<18.5(\mathrm{~N}))$ & $13 \%(8)$ & $11 \%(10)$ & 4.72 \\
\hline & \% Normal weight (BMI 18.5-25(N)) & $74 \%(47)$ & $65 \%(58)$ & \\
\hline & \% Overweight (BMI 25-30) & $13 \%(8)$ & $20 \%(18)$ & \\
\hline & $\%$ Obese $(\mathrm{BMI}>30)$ & $0 \%(0)$ & $4 \%(3)$ & \\
\hline
\end{tabular}

${ }^{*} \mathrm{P}<.01 ;{ }^{* *} \mathrm{P}<.001$ 


\section{Condition effects}

Change scores for the Intervention Group were compared with change scores for the control group for both behavioral outcome measures and determinants. All findings are presented in Table 2 .

Behavioral Outcomes. Results over time showed that there was a significantly bigger reduction in soft drink consumption in the intervention group $(\Delta M=-.15)$ as compared to the control group ( $\Delta M$ $=-.04)$. Moreover, the intervention group increased their use of active transport $(\Delta M=.65)$, while the use of active transport within the control group decreased $(\Delta M=-.12)$. We did not find significant intervention effects on fruit and vegetable intake, snack consumption, moderate PA or vigorous PA.

Determinants. Change scores for healthy DI indicated significant mean differences between the intervention and the control groups for attitude $\left(\Delta \mathrm{M}_{\text {Intervention Group }}=.-.08, \Delta \mathrm{M}_{\text {Control group }}=.02\right), \mathrm{SE}\left(\Delta \mathrm{M}_{\text {Interven- }}\right.$ tion Group $\left.=-.29, \Delta M_{\text {Control group }}=-.16\right)$, and action control $\left(\Delta M_{\text {Intervention Group }}=.51, \Delta M_{\text {Control group }}=.06\right)$. Results for unhealthy DI indicated no significant mean differences between the intervention and control groups. Results for PA indicated significant mean differences between the intervention and control groups in terms of intention $\left(\Delta \mathrm{M}_{\text {Intervention Group }}=-.31, \Delta \mathrm{M}_{\text {Control group }}=-.19\right)$ and action control $\left(\Delta \mathrm{M}_{\text {Intervention Group }}=.33\right.$, $\left.\Delta \mathrm{M}_{\text {control group }}=-.11\right)$, indicating that PA intentions within the intervention group decreased significantly more than in the control group. However, the intervention group showed increased action control skills (while these control skills decreased in the control group). 


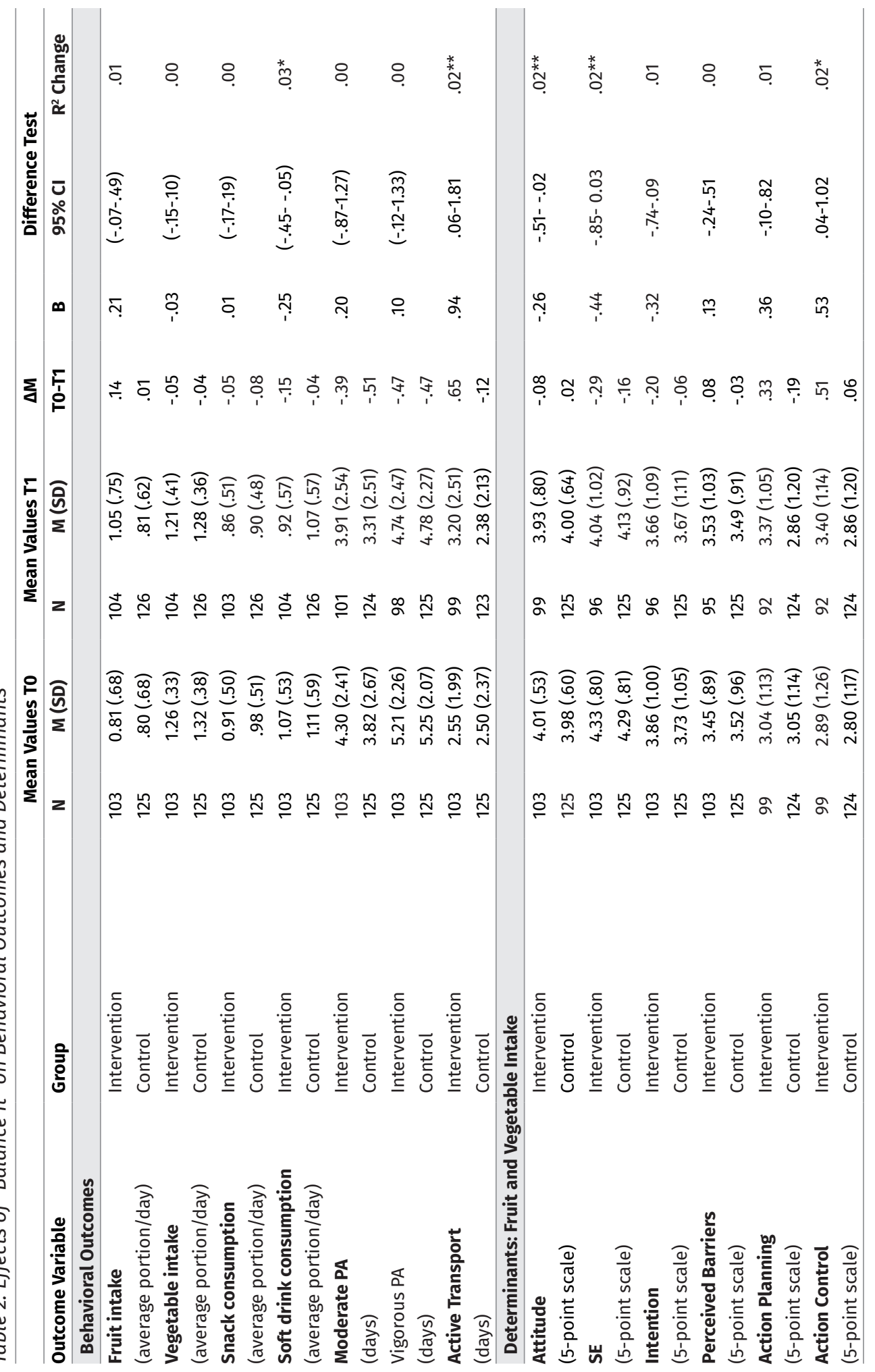




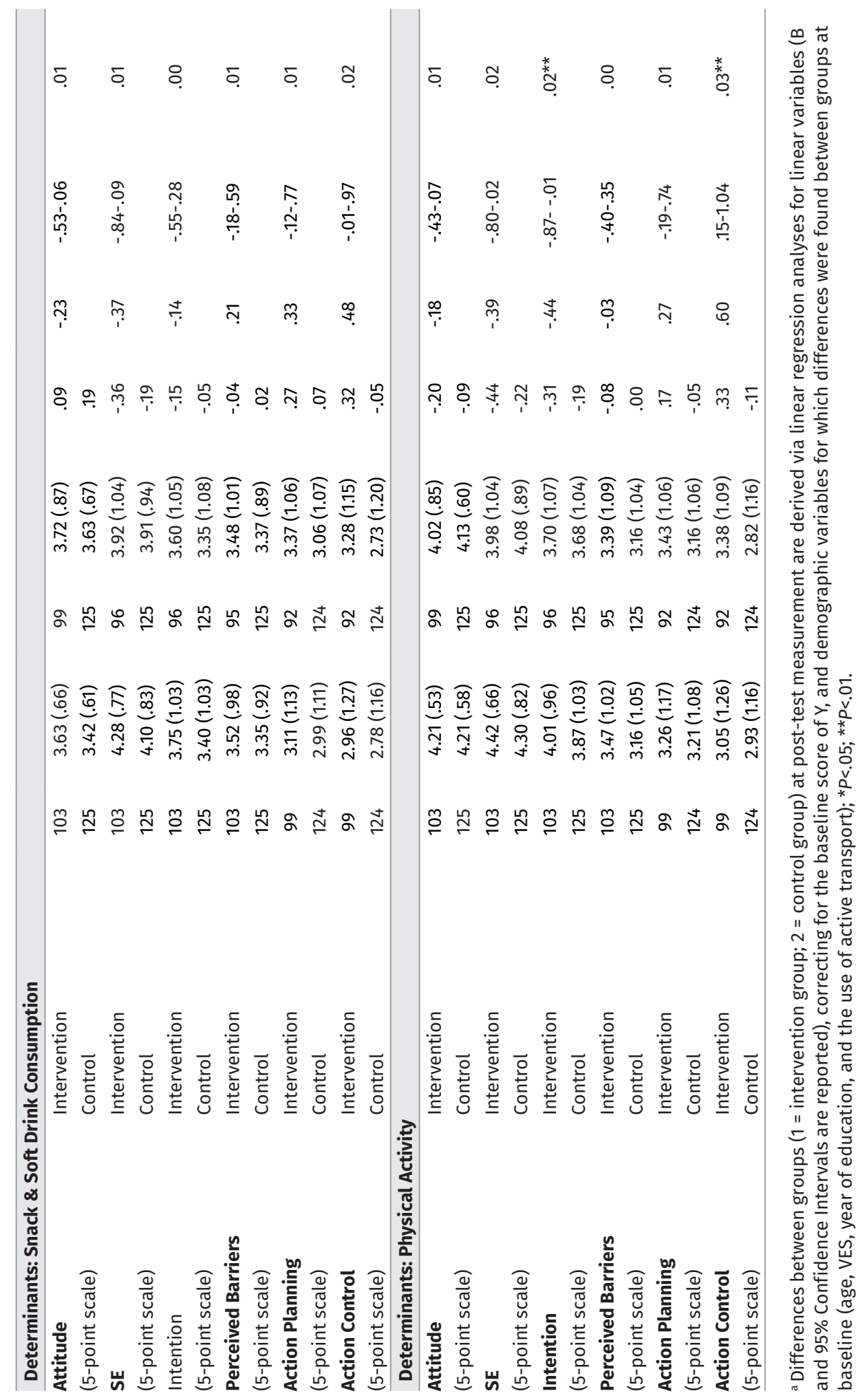




\section{Intervention users vs non-users}

The same regression analyses performed to compare the intervention and control groups were also used to compare the groups "intervention users" ( $N=29,28 \%$ intervention group) and "non-users" ( $N=200,72 \%$ intervention group $+100 \%$ control group). First, it should be noted that allocation to these groups was based on self-reported intervention use. Second, there were no significant differences between intervention users and non-users within the intervention group. As such, non-users from the intervention group and control group were merged and we tested for baseline differences to control for possible confounding effects of the group allocation.

Baseline differences. Compared to the non-users group, intervention users were more likely to participate in the VES "Economics" and less likely to participate in Care and well-being and Economy. Finally, they were more likely to follow the first year of secondary vocational education. We therefore controlled for these differences in the following analyses. We also controlled for baseline differences between the intervention users and non-users in case they differed significantly on behavioral outcomes and determinant measures. We found that the intervention users at baseline were more likely to use active transport as compared to the non-users $(P=.04)$. We therefore controlled for the use of active transport at baseline in all subsequent analyses concerning active transport.

Intervention use effects. Compared to non-users, intervention users reported increased fruit intake $\left(\Delta \mathrm{M}_{\text {Intervention Users }}=.51, \Delta \mathrm{M}_{\text {non-users }}=.06 ; \beta=.40, P=.003, \mathrm{R}^{2}\right.$ change $\left.=.04\right)$ and vegetable intake $\left(\Delta \mathrm{M}_{\text {Intervention }}\right.$ Users $=.06, \Delta \mathrm{M}_{\text {non-users }}=-.06 ; \beta=.15, P=.02, \mathrm{R}^{2}$ change $\left.=.03\right)$, and decreased snack consumption $\left(\Delta \mathrm{M}_{\text {Intervention }}\right.$ Users $=-.20, \Delta M_{\text {non-users }}=-.04 ; \beta=-.20, P=.02, R^{2}$ change $\left.=.03\right)$. The decrease in soft drink consumption was only marginally significant $\left(\Delta \mathrm{M}_{\text {Intervention Users }}=-.25, \Delta \mathrm{M}_{\text {non-users }}=-.07 ; \beta=-.17, P=.08, \mathrm{R}^{2}\right.$ change $\left.=.01\right)$, and there was no longer a significant difference in the use of active transport $\left(\Delta \mathrm{M}_{\text {Intervention Users }}=.92\right.$, $\Delta M_{\text {non-users }}=.13 ; \beta=.29, P=.50, R^{2}$ change $\left.=.00\right)$. Second, intervention users showed increased positive attitudes towards $\mathrm{PA}\left(\Delta \mathrm{M}_{\text {Intervention Users }}=.15, \Delta \mathrm{M}_{\text {non-users }}=-.17 ; \beta=.32, P=.01, \mathrm{R}^{2}\right.$ change $\left.=.03\right)$ as well as an increase in PA intentions $\left(\Delta \mathrm{M}_{\text {Intervention Users }}=.10, \Delta \mathrm{M}_{\text {non-users }}=-.39 ; \beta=.41, P=.05, \mathrm{R}^{2}\right.$ change $\left.=.02\right)$. Intervention users also reported enhanced action control skills for healthy eating $\left(\Delta M_{\text {Intervention Users }}=.88\right.$, $\Delta M_{\text {non-users }}=.14 ; \beta=.65, P=.01, R^{2}$ change $\left.=.04\right)$, unhealthy eating $\left(\Delta M_{\text {Intervention Users }}=.63, \Delta M_{\text {non-users }}=.02 ; \beta=\right.$ $.55, P=.02, R^{2}$ change $\left.=.03\right)$, and $P A\left(\Delta M_{\text {Intervention Users }}=.66, \Delta M_{\text {non-users }}=-.01 ; \beta=.64, P=.01, R^{2}\right.$ change $\left.=.04\right)$. 


\section{Process Evaluation of Self-Reported and Registered Game Play}

User data (an objective measure) showed that "Balance It" users played 1.7 games on average (total games played: $N=771)$. These games mainly consisted of daily tasks $(N=671 ; 87 \%)$ and individual game play $(N=632 ; 82 \%)$. Of all the goals set (i.e., type of tasks), players chose to improve their fruit intake in $15 \%$ of all cases ( $N=116$; of which $44 \%$ of the goals were accomplished $[N=51]), 3 \%$ opted to increase their vegetable intake ( $N=23$; of which $39 \%$ of the goals were accomplished [ $N=9]), 29 \%$ opted to decrease their snack consumption ( $N=224$; of which $70 \%$ of the goals were accomplished [N $=157]$ ), $9 \%$ opted to decrease their soft drink consumption ( $N=69$; of which $63 \%$ of soft drinks-related goals were accomplished $[N=44]), 31 \%$ opted to increase their moderate PA ( $N=239$; of which $55 \%$ of moderate PA goals were accomplished $[N=131])$, and $13 \%$ opted to increase their vigorous PA $(N=$ 100 ; of which $39 \%$ of vigorous PA goals were accomplished [ $N=39])$. Goal accomplishment was more likely when participants were motivated $(\mathrm{OR}=2.6,95 \% \mathrm{Cl} 1.88-3.54)$, and less likely when they did not have the time $(\mathrm{OR}=0.6,95 \% \mathrm{Cl} .43-.89)$ or when they experienced the location as a barrier $(\mathrm{OR}=0.6$, $95 \% \mathrm{Cl} .41-.94)$.

At post-test, $50 \%$ of the participants who used the intervention reported that they played "Balance It" because they wished to have a healthier lifestyle, and $50 \%$ played the game because they were asked to for the purpose of our study (total $N=29$ ). $24 \%$ of the participants who did not play "Balance It" mainly reported that they did not have the time to play $(N=18)$. The participants who used the intervention were, on average, neutral to positive about the "Balance It" app. When asked whether they were planning to recommend "Balance It" to others, participants gave a mean score of 3.14 (SD $=1.03$ ) on a scale ranging from 1 (very bad) to 5 (very good), and, likewise, the mean rating for the tutorial (using the same scale) was $3.72(S D=.75)$. Also, the specific game elements were evaluated neutrally to positively on average, ranging from $3.43(S D=1.00)$ on a scale ranging from 1 (very stupid) to 5 (very nice) for the construction worker, to $3.62(S D=.90)$ for the option of using special powers on the tower of an opponent. The mean overall rating given for the "Balance It" app (on a scale of 1 to 10 , with $1=$ the lowest grade and $10=$ the highest grade) was $6.71(S D=1.96)$. The mean overall rating for the website (using the same scale) was $6.5(S D=1.40)$. 


\section{Discussion}

The aim of the present study was to evaluate the effects of "Balance It", a serious game intervention targeting secondary vocational education students' dietary intake (DI) and physical activity (PA). In partial support of the hypotheses, the intervention group reported an average decrease in soft drink consumption of .15 glasses a day, and an average increase of .65 days a week using active transport. Taking into account that a difference of $100 \mathrm{kcal}$ in daily caloric intake/expenditure can contribute to overweight prevention (Hill, Wyatt, Reed, \& Peters, 2003), decreased soft drink consumption and increased active transportation contribute marginally to the prevention of overweight. Furthermore, action control skills increased for healthy DI and PA as compared to the control group. No effects were found for fruit and vegetable intake, snack consumption, or PA. Contradictory to our hypotheses, the intervention group showed less positive attitudes towards healthy DI, decreased SE in relation to healthy $\mathrm{DI}$, and decreased PA intentions at post-test measurement. However, process evaluation data showed that only $28 \%$ of the intervention group reported actual use of "Balance It", and this subset of participants rated the intervention moderately positively. The following comparison between intervention users and non-users showed more support for our hypotheses, indicating increased fruit consumption (an increase of .51 pieces a day on average) and vegetable consumption (an increase of .06 portions a day on average) and decreased snack consumption (-.20 portions a day on average). Although intervention users reported a decrease in soft drink consumption of -.25 glasses a day on average, this result was only marginally significant. There were no significant differences in the use of active transport when comparing intervention users with non-users. The determinants PA attitude, PA intentions, and action control skills for both DI and PA increased among intervention users. Registered "Balance It" data showed that users mainly opted to decrease their snack consumption (29\%) or to increase their moderate PA (31\%).

Consistent with previous serious gaming studies targeting DI and PA, our results suggest that the use of a self-regulation game intervention could improve DI among youth (Baranowski et al., 2000; Baranowski et al., 2011). Despite the significant increase in use of active transport for the intervention condition, intervention users did not report a significant increase in active transport, or moderate or vigorous PA. That we found no direct effect of the intervention on PA may be due to the fact that post-test measures took place after four weeks of game play, whereas PA goals were partially set on a weekly basis, taking up to six weeks of game play, and were therefore not yet completed at the time of the post-test measure. The four-week period may also be too short to expect change in PA (Prochaska, 2013). 
Unexpectedly, despite favorable changes in behavior, results showed less positive attitudes and lower SE relating to healthy DI at the post-test measure, which may indicate that "Balance It" has a negative effect on students' DI attitude and SE. It should be noted that retrospective reporting on complex behaviors such as PA is relatively difficult, and that these findings may be confounded by a so-called response-shift bias, indicating that the results may reflect a change in awareness or understanding of their PA rather than an actual change in PA (Howard \& Dailey, 1979). Registered data showed that $44 \%$ of the goals that were set by the intervention users focused on the improvement of PA, suggesting that participants' intended to increase their levels of PA. However, as lack of motivation and time were often identified as barriers, these barriers might have resulted in difficulties in continued intervention use, or in motivation and the sustainability of behavior change. As our aim was to increase intrinsic motivation for intervention use by creating enjoyment through serious gaming, future research should address how enjoyable the target population considers different elements of the game to be.

Previous research shows that youth from low socioeconomic status (SES) families are less engaged with health behaviors and not as successful in terms of translating their health intentions into behavior (Conner et al., 2013). We, therefore, incorporated gameplay, reminders, professional support, and social contracting into our serious game to stimulate intervention use. Moreover, we plan to embedded the intervention within an existing student tracking system as suggested by Crutzen, Kohl, and de Vries (2013; Abraham \& Michie, 2008; Brouwer et al., 2011; Crutzen et al., 2013; and Spook et al., 2015 for a more detailed description of IM step 5: program implementation), to stimulate initial use. Despite these attempts to encourage implementation use, only $28 \%$ of the intervention group reported actual use of "Balance It". Although the participants who used the intervention were moderately positive about "Balance It", it may be that players were not sufficiently transported into the game by the narrative of "Balance It" because the narrative that was used to stimulate immersion is more in line with what Lu, Baranowski, Thompson, and Buday (2012) describe as an "instruction", which does not adequately facilitate immersion (Lu, Baranowski, Thompson, \& Buday, 2012). According to Lu et al., narratives should have attractive features: a plot, a beginning, middle, and an end, and should allow players to experience a character's happiness on their journey towards adoption of a healthy behavior more directly and vividly than didactic instruction alone (Lu et al., 2012). However, in practice, professional game designers often stress the importance of game simplicity to enhance motivation to play (see also Fogg, 2009), limiting the space for extensive narratives to be included 
within a game. One way of resolving this issue would be to use so-called "novellas", which can be defined as highly immersive stories designed to increase engagement with the game intervention provided prior to the game (Baranowski \& Baranowski, 2015). Placing novellas outside the "Balance It" app may also be beneficial in terms of exposure, as students were stimulated to follow the link to the "Balance It" website by the research assistant during pre-test, but the research assistant was not able to check if all students downloaded the app. As such, participants would be more likely to be exposed to the novella, increasing the likelihood of intervention (or "Balance It" app) use. However, these so called "novellas" are still in their infancy, but as they seem to be a rather promising way of countering low levels of engagement, further research is wishful.

\section{Limitations of the present study}

Some limitations of the present study should be acknowledged. First, the study is a Group-Randomized Trial (GRT), which was chosen over a randomized controlled trial (RCT) because of practical considerations (i.e., school coordinators who wanted their students to be in the same condition), to prevent contamination effects, and to enhance participant compliance (Donner \& Klar, 2000; McKenzie, Ryan, Di Tanna, \& Cochrane Consumers and Communication Review Group, 2014). Despite these efforts, however, contamination effects may have influenced the results of the comparison between intervention users and non-users, as about $48 \%$ of the non-users were exposed to the intervention). It should also be noted that participants who were at criteria for the outcomes at baseline were included in the GRT, which may have reduced the overall effect size.

A second limitation is that only a pre-test and post-test were included in the present study, and follow-up measures to evaluate long-term effects were lacking. Consequently, the results of the present study are based on change scores collected over a four-week period, while many PA related goals that were set by game players took six week to accomplish. As such, we might not expect the full effects of "Balance It" to reveal themselves until participants had used it for six weeks. Participants were contacted via email and requested to fill-in the follow-up questionnaire four weeks after post-test measure, but with a response rate of only $4 \%(N=20)$, we did not analyze this data. Finally, email addresses were inaccurately reported (or not reported) in the "Balance It" app, which was unfortunate as we needed them to merge (objective) user data with (self-reported) survey data at baseline and at post-test. Consequently, user data could not be merged with self-reported data and conclusions about the objective use of the intervention in relation to the learning outcomes should 
therefore be interpreted with care. We recommend that future research uses a RCT design and incorporates appropriate follow-up measures and checks regarding the collection of email addresses or the inclusion of other merge variables.

Finally, it should be noted that despite the potential of the peer-support component as included in the "Balance It" website to increase intervention effects on self-regulation skills (Heisler, 2009), usage of the peer-support system was rather disappointing, (i.e., only $7 \%$ of the intervention users reported actual use of the peer-support system). The lack of peer-support system use is a commonly reported problem in online interventions (Eysenbach et al., 2004). As the peer-support system was placed outside the "Balance It" app as a result from the trade-off that was made between developers and behavior change experts about the number of layers within the game, visiting the system online may have been a barrier. A second explanation for the lack of peer-support system use may also be derived from the limited number of schools (i.e., four) included in the present GRT. In total, only two schools were allocated to the intervention group, indicating that most participants knew each other in daily life. So in case students preferred to receive or provide social support, this may have taken place in real life instead of through the online peer-support system of "Balance It".

\section{Conclusion}

The current effect evaluation of "Balance It" contributes to our overall understanding of changing DI and PA behaviors and the role that serious games can play in changing these behaviors. As intervention use did not reach optimal levels in the present study, we recommend that future research assesses the influence of "Balance It" once the intervention has been embedded within an existing student tracking system. In conclusion, "Balance It" shows promise for the further development and use of serious games to target health-related behaviors among youth.

\section{Conflicts of Interest}

None declared. 


\section{Abbreviations}

DI: Dietary Intake

PA: Physical Activity

SES: Socioeconomic Status

BMI: Body Mass Index

VES: Vocational Education Sector

RCT: Randomized Controlled Trial

$\Delta$ : Difference Score

$\Delta M$ : Mean Difference 


\section{References}

Abraham, C., \& Michie, S. (2008). A taxonomy of behavior change techniques used in interventions. Health Psychology, 27(3), 379-387.

Araújo-Soares, V., McIntyre, T., \& Sniehotta, F. F. (2009). Prediciting changes in physical activity among adolescents: The role of self-efficacy, intention, action planning and coping planning. Health Education Research, 24(1), 128-139.

Assema van, P., Brug, J., Ronda, G., \& Steenhuis, I. (2001). The relative validity of a short dutch questionnaire as a means to categorize adults and adolescents to total and saturated fat intake. The Britisch Dietetic Association, 14, 377-390.

Baranowski, T., \& Baranowski, J. (2015). Using fictional novellas as narrative companion for vegetable parenting game. Proceedings of the International Society of Nutritional Behavior and Physical Activity: Advancing Behavior Change Science. Edinburgh.

Baranowski, T., Baranowski, J., \& Cullen, K. W. e. a. (2003). Squire's quest! dietary outcome evaluation of a multimedia game. American Journal of Preventive Medicine, 24(1), 52-61.

Baranowski, T., Baranowski, J., Thompson, D., Buday, R., Jago, R., Juliano Griffith, M., ... Watson, K. B. (2011). Video game play, child diet, and physical activity behavior change: A randomized clinical trial. American Journal of Preventive Medicine, 40(1), 33-38.

Baranowski, T., Buday, R., Thompson, D. I., \& Baranowski, J. (2008). Playing for real: Video games and stories for health-related behavior change. American Journal of Preventive Medicine, 34(1), 74-82.

Baranowski, T., Davis Hearn, M., Resnicow, K., Baranowski, J., Doyle, C., Lin, S., . . Wang, D. T. (2000). Gimme 5 fruit, juice, and vegetables for fun and health: Outcome evaluation. Health Education Behaviour, 27(1), 96-111.

Bartholomew, L. K., Parcel, G. S., Kok, G., Gottlieb, N. H., \& Fernández, M. E. (Eds.). (2011). Planning health promotion programs: An intervention mapping approach. San Francisco: Jossey-Bass.

Bernaards, C., \& Buuren van, S. (2012). Rapportage veranderingen in het beweeggedrag van mbo studenten. (No. R10185). Leiden, the Netherlands: TNO Behavioural and Societal Sciences. 
Brouwer, W., Kroeze, W., Crutzen, R., de Nooijer, J., de Vries, N., Brug, J., \& Oenema, A. (2011). Which intervention characteristics are related to more exposure to internet-delivered healthy lifestyle promotion interventions? A systematic review. Journal of Medical Internet Research, 13(1), e2.

Conner, M., McEachan, R., Jackson, C., McMillan, B., Woolridge, M., \& Lawton, R. (2013). Moderating effect of socioeconomic status on the relationship between health cognitions and behaviors. Annals Behavioral Medicine, 46, ’19-30. doi:10.1007/a12160-013-9481-y

Crutzen, R., De Nooijer, J., Brouwer, W., Oenema, A., Brug, J., \& De Vries, N. K. (2008). Internet-delivered interventions aimed at adolescents: A delphi study on dissemination and exposure. Health Education Research, 23, 427-439.

Crutzen, R., de Nooijer, J., Brouwer, W., Oenema, A., Brug, J., \& de Vries, N. K. (2011). Strategies to facilitate exposure to internet-delivered health behavior change interventions aimed at adolescents or young adults: A systematic review. Health Education Behaviour, 38(1), 49-62.

Crutzen, R., Kohl, L., \& De Vries, N. K. (2013). Kennissynthese online preventie

DeSmet, A., Van Ryckeghem, D., Compernolle, S., Baranowski, T., Thompson, D., Crombez, G., ... De Bourdeaudhuij, I. (2014). A meta-analysis of serious digital games for healthy lifestyle promotion. Preventive Medicine, 69, 95-107.

Donner, A., \& Klar, N. (2000). Design and analysis of cluster randomization trials in health research. London: Arnold Publishing.

Douwes, M., \& Hildebrandt, H. (2000). Vragen naar de mate van lichamelijke activiteit. Geneeskunde En Sport, 33(1), 9-16.

Eisenberg, M. E., Neumark-Sztainer, D., \& Story, M. (2003). Associations of weight-based teasing and emotional well-being among adolescents. Archives of Pediatrics \& Adolescent Medicine, 157, 733-738.

Eysenbach, G., Powell, J., Englesakis, M., Rizo, C., \& Stern, A. (2004). Health related virtual communities and electronic support groups: Systematic review of the effects of online peer to peer interactions. British Medical Journal, 328, 1166.

Fogg, B. J. (2009). A behavior model for persuasive design. Proceedings of the 4th International Conference on Persuasive Technology, ACM. 
Formiguera, X., \& Canton, A. (2004). Obesity: Epidemiology and clinical aspects. Best Practice and Research Clinical Gastroenterol, 18(6), 1125-1146.

Graesser, A., Chipman, P., Leeming, F., \& Biedenback, S. (2009). Deep learning and emotion in serious games. In U. Ritterfeld, M. Cody \& P. Vorderer (Eds.), Serious games, mechanisms and effects. (). New York and London: Routledge.

Heisler, M. (2009). Different models to mobilize peer support to improve diabetes self-management and clinical outcomes: Evidence, logistics, evaluation considerations and needs for future research. Family Practice, , 1-10.

Hill, J. O., Wyatt, H. R., Reed, G. W., \& Peters, J. C. (2003). Obesity and the environment: Where do we go from here? Science, 299(5608), 853-855.

Howard, G. S., \& Dailey, P. R. (1979). Response-shift bias: A source of contamination of self-report measures. Journal of Applied Social Psychology, 64(2), 144-150.

Krebs, P., Prochaska, J. O., \& Rossi, J. S. (2010). A meta-analysis of computer-tailored interventions for health behavior change. Preventive Medicine, 51, 214-221.

Kroeze, W., Werkman, A., \& Brug, J. (2006). A systematic review of randomized trials on the effectiveness of computer-tailored education on physical activity and dietary behaviors. Annals of Behavioral Medicine, 31(3), 205-223.

Lu, A. S., Baranowski, T., Thompson, D., \& Buday, R. (2012). Story immersion of videogames for youth health promotion: A review of literature. Games for Health Journal: Research, Development, and Clinical Applications, 1(3), 199-204.

Maes, S., \& Karoly, P. (2005). Self-regulation assessment and intervention in physical health and illness: A review. Applied Psychology: An Internation Review, 54, 267-299.

McKenzie, J., Ryan, R., Di Tanna, G. L., \& Cochrane Consumers and Communication Review Group. (2014). Cochrane consumers and communication review group: Cluster randomised controlled trials. (). http:// cccrg.cochrane.org/sites/cccrg.cochrane.org/files/uploads/ClusterRCTs.pdf: Cochrane Consumers and Communication Review Group. 
Nguyen, B., Kornman, P., \& Baur, L. A. (2011). A review of electronic interventions for prevention and treatment of overweight and obesity in young people. Obesity Reviews, 12, e298-e314.

Prensky, M. (2007). Digital game-based learning. St. Paul, Minnesota: Paragon House.

Prochaska, J. O. (2013). Transtheoretical model of behavior change. In M. D. Gellman, \& J. R. Turner (Eds.), Encyclopedia of behavioral medicine (pp. 1997-2000). New York: Springer.

Rijpstra, A., \& Bernaards, C. (2011). De leefstijl van MBO studenten in nederland 2009/2010. (No. TNO/ LS 2011.014). Leiden, the Netherlands: TNO.

Roberts, R. E., Deleger, S., Strawbridge, W. J., \& Kaplan, G. A. (2003). Prospective association between obesity and depression: Evidence from the alameda county study. International Journal of Obesity Related Metabolism Disorders, 27(4), 514-521.

Schönbeck, Y., \& Buuren van, S. (2010). Factsheet resultaten vijfde landelijke groeistudie. TNO Leiden, Sniehotta, F. F., Nagy, G., Scholz, U., \& Schwarzer, R. (2006). The role of action control in implementing intentions during the first weeks of behaviour change. British Journal of Social Psychology, 45, 87-106.

Spook, J. E., Paulussen, T., Kok, G., \& van Empelen, P. (2013). Monitoring dietary intake and physical activity electronically: Feasibility, usability, and sensitivity of a mobile-based ecological momentary assessment (mEMA) tool. Journal of Medical Internet Research, 15(9), e214.

Spook, J. E., Paulussen, T., Kok, G., \& van Empelen, P. (2015). Design rationale behind the serious self-regulation game intervention "balance it": Overweight prevention among secondary vocational education students in the netherlands. Games for Health Journal: Research, Development, and Clinical Applications, 4(6) doi:10.1089/g4h.2014.0142

Spook, J. E., Paulussen, T., Kok, G., \& van Empelen, P., submitted for publication. Factors determining physical activity of emerging young adults: A mobile-based Ecological Momentary Assessment (mEMA) study

Spook, J. E., Paulussen, T., Kok, G., \& van Empelen, P., submitted for publication. The role of motivational and contextual factors on intentional and craving-induced snack consumption: A mobilebased ecological momentary assessment study. 
Statistics Netherlands. (2000). Hoe doet het CBS dat nou? STandaarddefinitie allochtonen [how does statistics netherlands do this? standard definition immigrants]. (No. 10). Voorburg: Statistics Netherlands.

Storch, E. A., Milsom, V. A., DeBraganza, N., Lewin, A. B., Geffken, G. R., \& Silverstein, J. H. (2007). Peer victimization, psychosocial adjustment and physical activity in overweight and at-risk-for-overweight youth. Journal of Pediatric Psychology, 32(1), 80-89.

Stunkard, A. J., Faith, M. S., \& Allison, K. C. (2004). Depression and obesity. Biological Psychiatry, 54(3), 330-337.

Tate, D. F. (2008). Application of innovative technologies in the prevention and treatment of overweight in children and adolescents. In E. Jelalian, \& R. G. Stelle (Eds.), Handbook of childhood and adolescent obesity. (pp. 378-404). New York: Springer Science and Business Media.

Thompson, D., Bhatt, R., Vazquez, I., Cullen, K. W., Baranowski, J., Baranowski, T., \& Liu, Y. (2015). Creating action plans in a serious video game increases and maintains child fruit-vegetable intake: A randomized controlled trial. International Journal of Behavioral Nutrition and Physical Activity, 12(39), 1-10.

Van der Horst, K., Oenema, A., Van de Looij-Jansen, P., \& Brug, J. (2008). The ENDORSE study: Research into environmental determinants of obesity related behaviors in rotterdam schoolchildren. BMC Public Health, 8, 142.

Van Genugten, L., Van Empelen, P., Flink, I., \& Oenema, A. (2010). Systematic development of a self-regulation weight-management intervention for overweight adults. BMC Public Health, 10, 649.

Van Genugten, L., Van Empelen, P., \& Oenema, A. (2014). Intervention use and action planning in a web-based computer-tailored weight management program for overweight adults: Randomized controlled trial. Journal of Medical Internet Research, 3(3), e.31.

Van Keulen, H. M., Mesters, I., Ausems, M., van Breukelen, G., Campbell, M., Resnicow, K., ... de Vries, H. (2011). Tailored print communication and telephone motivational interviewing are equally successful in improving multiple lifestyle behaviors in a randomized controlled trial. Annual Behavioral Medicine, 41, 104-118. 
Van't Riet, J., Crutzen, R., \& de Vries, H. (2010). Investigating predictors of visiting, using, and revisiting an online health-communication program: A longitudinal study. Journal of Medical Internet Research, 12(3), e37.

Wang, Y., Liang, H., Tussing, L., Braunschweig, C., Cabellero, B., \& Flay, B. (2007). Obesity and related risk factors among low socio-economic status minority students in chicago. Public Health Nutrition, 10(9), 927-938.

Wang, Y., Monteiro, C., \& Popkin, B. M. (2002). Trends of obesity and underweight in older children and adolescents in the united states, brazil, china, and russia. The American Journal of Clinical Nutrition, 75, 971-977.

World Health Organization. (2004). Diet, nutrition and the prevention of chronic diseases. (). Geneva, Switzerland: WHO. 



\section{CHAPTER 7}

General Discussion 


\section{General Discussion}

This final chapter of this dissertation aims to provide an overview of our main findings, to critically reflect on these findings, and to provide directions for future research. Previous research has shown that overweight-related behaviors (ORBs; i.e., dietary intake [DI] and physical activity [PA]) are both difficult to understand and change, and hence a challenge to overcome. Therefore, our specific aims were: 1) to gain a better understanding of secondary vocational education students' ORBs in realtime settings, and 2) to examine whether serious gaming can change these behaviors. In the studies presented in this dissertation, we applied two novel methods (i.e. mobile-based Ecological Momentary Assessment (mEMA) and serious gaming) in order to understand and change ORBs in the daily life of Dutch secondary vocational education students.

In "PART I" we specifically focused on gaining a better understanding of ORBs by means of " $m E M A$ ". To this end, Chapter 2 focused on the feasibility and usability of "mEMA". This was followed by Chapters 3 and 4 which explored the motivational and contextual factors that are associated with ORBs both within a single time slot and cross-time. In "PART II", our goal was to change secondary vocational education students' DI and PA. First, in Chapter 5, we described the design rationale of "Balance It", a serious game intervention targeting OCBs, and finally Chapter 6 consisted of an evaluation of "Balance It". "PART III" described the promises and pitfalls of applying the Intervention Mapping (IM) protocol in game-based intervention studies. Finally, we added a "PART IV" to this General Discussion, describing characteristics of the targeted population (i.e., secondary vocational education students), practical constraints of mHealth research, implications for research and practice, and directions for future research directions.

\section{PART I: Understanding Overweight-Related Behaviors in Daily Life}

Over several decades, researchers have tried to identify which factors influence the two main overweight-related behaviors: dietary intake (DI) and physical activity (PA). However, due to their complex and repetitive nature, measuring these behaviors has been quite problematic in cross-sectional research. Two of the main challenges of using retrospective self-reports to measure these behaviors are the inaccuracy of these kinds of measures due to methodological issues (e.g., recall bias; Stone, Shiffman, \& de Vries, 2000), and the influence of alternative, environmental factors on DI and PA. In the present dissertation, we addressed these issues by utilizing mobile-based Ecological Momentary Assessment (mEMA). This method enables repetitive data collection in real-time, natural settings 
and can overcome problems such as faked compliance, backfilling, and deficient data which were more common among precursors of EMA research (Broderick, Schwartz, Shiffman, Hufford, \& Stone, 2003; Stone, Shiffman, Atienza, \& Nebeling, 2007). Information and communication developments offer new ways in which to study complex behaviors. This, coupled with the increase of smartphone use among the target population ( $89 \%$ of the targeted secondary vocational education students possessed a smartphone [10\% a cell-phone, and $1 \%$ unknown], and were likely to use their smartphone on a daily basis (Spook, Paulussen, Kok, \& van Empelen, 2013; SURFnet, 2013), means that mEMA research is assumed to fit well into daily life, and to capture ecologically valid, momentary data.

Additionally, one of the benefits of using mEMA is that it is possible to send prompts, allowing researchers to remind participants to use the tool in order to enhance participant compliance (Stone et al., 2003). As non-compliance is a familiar challenge in e-/mHealth research, we implemented seven behavioral science principles prior to the study in order to enhance compliance to EMA protocols. These principles were: 1) facilitating and rewarding participant compliance, 2) participant training, 3) using easy-to-use interfaces, 4) "driving" the protocol by real-time compliance reminders, 5) guiding subjects through assessments, 6) incorporating "livability" functions such as suspension options for time caps in which reminders might be inappropriate (e.g., at school), and 7) creating a sense of accountability for the data (Hufford \& Shiffman, 2003). As such, we aimed to develop a feasible and usable mEMA tool (called "mEMA") to measure secondary vocational education students' ORBs and associated factors in daily life. The following paragraphs (1.1. "Real Time Assessment of Dietary Intake and Physical Activity" and 1.2. "Real Time Assessment of Associated Factors of Dietary Intake and Physical Activity") outline the main findings of the mEMA feasibility study (Chapter 2), the assessment of real-time DI (Chapter 3 ) and PA (Chapter 4), and the factors associated with these behaviors. Finally, a critical reflection of the research carried out, a discussion of methodological issues and recommendations for future research will be outlined.

\subsection{Real Time Assessment of Dietary Intake and Physical Activity}

The main findings of the feasibility and usability study presented in Chapter 2 showed that the target population (i.e., secondary vocational education students) generally carried their smartphone around with them on a daily basis, that "mEMA" was easy to use, and that the questions were well-displayed on their smartphones, indicating that mEMA is a feasible and easy to use means of studying complex 
behaviors such as DI and PA (Spook et al., 2013). Subsequently, in Chapters 3 and 4, we explored one of the most promising aspects of real-time data capturing -- i.e. how behaviors unfold over time (Stone et al., 2007). In accordance with previous research on DI and PA by Wouters et al. (2013) and Wichers et al. (2012; 2013), we showed that snack consumption (SC) and PA fluctuate during the day. SC was most likely to occur at the end of the day, whereas PA was most likely to take place during the late afternoon (Spook, Paulussen, Kok, \& van Empelen, submitted for publication; Spook, Paulussen, Kok, \& van Empelen, submitted for publication).

Bearing in mind that survey studies generally rely on (biased) self-reported measures of average DI and/or PA, it is questionable which DI or PA event participants reflect upon in survey studies, and how accurate these self-reflections are. Recall bias is a common methodological issue in retrospective self-reports (Stone et al., 2000), and may also influence self-reported compliance rates (Stone et al., 2007; Stone \& Shiffman, 2002). As such, we compared registered compliance and self-rated compliance in all three mEMA studies. An overall comparison of registered mEMA compliance (43.8\%) showed that students overestimated their compliance by approximately $26 \%$ as compared to average self-reported compliance data (70.6\%). Although most EMA studies to date have not reported actual compliance rates, Haedt-Matt and Keel reported compliance rates of $80-85 \%$ in their meta-analysis (Haedt-Matt \& Keel, 2011). These rates are double the average compliance rate found across the three mEMA studies presented in this thesis.

This difference might be explained by the targeted population (i.e., secondary vocational education students in the Netherlands), as lower compliance rates are more commonly reported among less educated students (Heron \& Smyth, 2010). As such, these students may be more difficult to reach. Although initial mEMA use was $90 \%$ on average and thus acceptable, continued use was rather poor (i.e., there was an average decrease of 58\% over the three mEMA studies) and could therefore be considered the main challenge of mEMA research.

As the poor use of mEMA, or noncompliance, can influence the results of a study (Stone et al., 2007), it is vital to understand which factors underlie the lack of continued mEMA use and how this use might be increased. First, compliance is subject to the hassles of everyday life and natural human forgetfulness (Stone et al., 2007). As such, adolescents may struggle to complete EMA surveys due in part to the chaotic environment in which they are prompted (Marszalek, Morgulex-Adamowicz, Rutkowska, \& 
Kosmol, 2014). Therefore, deliberate choices should be made in terms of signal-contingent recording, in which interference with daily activities should be taken into account.

Second, the use of a large number of prompts and reminders in case they were missed may result in reporting fatigue. Participants can get tired or annoyed (i.e., experience monitoring burden), and this has been shown to be significantly correlated with the number of daily prompt entries (Stone, Shiffman, Schwartz, Broderick, \& Hufford, 2003). However, recall bias may increase simultaneously with any increase in time between prompt intervals (i.e., time slots). As such, the accuracy of momentary data may decrease, which is problematic as this accuracy is one of the advantages of mEMA data gathering. So although having too-frequent prompts may be perceived as burdensome in an adolescent population, too-few prompts may lead to participants disengaging from the mEMA tool and forgetting to attend to the protocol (Sokolovsky, Mermelstein, \& Hedeker, 2014). Therefore, inter prompt-interval decisions during the developmental process of mEMA should be based on a trade-off between monitoring burden and disengagement. To this end, we piloted the level of monitoring burden in the feasibility study (see Chapter 2). Results showed that students perceived receiving five prompts a day, during eight consecutive days, as burdensome. Subsequently, we reduced the number of prompts to four times a day (i.e., morning, around noon, late afternoon, and evening) for seven consecutive days, aiming to decrease monitoring burden, and in turn, to increase mEMA use. However, as registered compliance rates remained disappointingly low, it may well be worthwhile incorporating other techniques designed to increase motivation in future mEMA research in order to enhance usage.

\subsection{Real Time Assessment of Factors Associated With Dietary Intake and Physical Activity}

Overweight-related behaviors (ORBs) are commonly explained from a socio-cognitive perspective (e.g., Theory of Planned Behavior; Ajzen, 1991). From this perspective, behavioral intentions are seen as the dominant predictor of complex health behaviors. However, it has been shown that these intentions only predict approximately $20 \%$ of actual behavior, and even less regarding PA in youth (i.e., 12\%, see McEachan, Conner, Taylor, \& Lawton, 2011; Motl et al., 2002). Moreover, among low SES groups, the association between intention and behavior is expected to be even weaker (Conner et al., 2013), which may indicate that they are more vulnerable to environmental influences and/or less conscious processes of behavioral decision-making (cf the EnRG framework, which suggests that health behavior is the result of an interplay between intrapersonal and environmental factors 
(Kremers et al., 2006; Sallis, Johnson, Calfas, Caparosa, \& Nichols, 1997; Spence \& Lee, 2003)). We therefore measured both intrapersonal and environmental factors associated with snack consumption (SC) and physical activity (PA) in daily life.

The main findings of Chapters 3 and 4 showed that motivational factors (including intention) were unrelated to SC and PA within singular time-slots, but were associated with subsequent SC and PA. These findings are in line with a meta-analysis conducted by McEachan, Conner, Taylor, and Lawton (2011), who showed that Perceived Behavioral Control (PBC) and intention explained 21.2\% of prospective dietary intake (DI), and $23.9 \%$ of prospective PA (McEachan et al., 2011). Interestingly, McEachan et al. also analyzed interaction effects between DI and PA and age, revealing that among students, these cognitive factors could explain up to $29.7 \%$ of PA, but only $9.6 \%$ of DI, indicating that DI among students may predominately be driven by other factors, such as impulsive reactions and environmental factors.

Bearing in mind that the intention-behavior relationship has been shown to be weaker among individuals from socioeconomically disadvantaged groups (Conner et al., 2013), the secondary vocational education students were expected to be more vulnerable to both impulsive reactions and environmental influences. In Chapter 3, we showed that food craving was strongly associated with SC within a specific time slot (but not over time), which may indicate that food craving has a stronger influence on SC than snack intentions, hampering these students' attempts to withstand tempting food stimuli from the obesogenic environment as their self-control also becomes depleted over time (Hagger, Wood, Stiff, \& Chatzisarantis, 2010). However, results also showed that environmental factors such as the presence of others also contributed to the prevention of SC. It may be that the social context consisted of unfamiliar co-eaters, as Salvy, Jarrin, Paluch, Irfan, and Pliner (2007) showed that adolescents adjust their level of eating as a function of the familiarity with their co-eater, and that both male and female adolescents ate less in the presence of unfamiliar people compared to adolescents who ate in the presence of familiar co-eaters (Salvy, Jarrin, Paluch, Irfan, \& Pliner, 2007). However, research has also shown that average-weight girls are more likely to show unhealthy weight-control behaviors, such as fasting, based on social norms within their peer group (Eisenberg, Neumark-Sztainer, Story, \& Perry, 2005). As such, students may have been in the social presence of friends, which may have hampered their SC. In order to gain a better understanding of social influences on secondary vocational education students' SC, future research should focus on how the presence of 
specific types of relationships facilitate or hamper students' SC.

In sum, mEMA can be considered to be a feasible and useful method to study complex health behaviors in real life settings. We showed that overweight-related behaviors fluctuate during the day, and more specifically that they are influenced by intrapersonal factors over time and environmental factors within specific time slots. Snack intentions seem to predict subsequent SC, but within specific situations, SC is most likely to occur when students experience feelings of food craving. Still, it should be noted that although the use of mEMA can decrease methodological issues such as recall bias, the three mEMA studies conducted in the present thesis were still hampered by low compliance rates despite the prompting technique we incorporated. As such, we recommend that more techniques designed to motivate participants are applied to future applications in order to stimulate the use of mEMA tools.

\section{PART II: Changing Overweight-Related Behaviors in Daily Life}

In the second part of this dissertation, we focused on changing overweight-related behaviors (ORBs) in daily life by use of a serious game, "Balance It". Up to date, multiple online health promotion interventions have been developed, but they have been hampered by small and varying effect sizes (Crutzen, Kohl, \& de Vries, 2013; Krebs, Prochaska, \& Rossi, 2010; Kroeze, Werkman, \& Brug, 2006; Lobstein, Baur, \& Uauy, 2004; Verweij, Coffeng, van Mechelen, \& Proper, 2011; Webb, Joseph, Yardley, \& Michie, 2010). These limited effect sizes have repeatedly been associated with high drop-out rates and non-compliance (Douketis, Maci, Thabane, \& Williamson, 2005; van Genugten, 2011), indicating a possible lack of motivation for behavior change, which is also more common among socioeconomically disadvantaged groups (Hardcastle et al., 2015). For example, in terms of PA, up to $30 \%$ of individuals express no intention to exercise, are not motivated to engage in health-promoting behaviors and, therefore, tend to be those most at risk for health issues (Hardcastle et al., 2015; Rhodes \& de Bruijn, 2013; Ronda, van Assema, \& Brug, 2001). As such it is very important to find ways of increasing intrinsic motivation to engage with health promotion interventions.

One promising strategy to increase the use of interventions is serious gaming. Serious games have the potential to be highly motivating, enjoyable, attention-captivating, and highly interactive. Moreover, they are thought to increase self-efficacy and have a positive effect on healthy lifestyle behaviors and their determinants (DeSmet et al., 2014; Krebs et al., 2010). However, in terms of serious 
game based solely on gaming or behavior change theory, effect sizes remain relatively small and these kinds of interventions may be considered to be just as effective as other computer-delivered interventions (DeSmet et al., 2014; Krebs et al., 2010). Nevertheless, serious gaming interventions are assumed to benefit from a dual theoretical foundation in both health promotion and gaming. This merging of theoretical fields is gaining more and more ground, and stimulates a dialogue between different research groups in order to create "the best of both worlds" (Crutzen, 2014; DeSmet et al., 2014).

\subsection{Serious Gaming to Promote Healthy Dietary Intake and Physical Activity}

The "Balance It" intervention was mainly grounded in Self-Regulation Theory (SRT) with the aim of promoting a healthy lifestyle (i.e., through goal setting, self-monitoring, barrier identification and behavioral feedback), and the RE-AIM model in order to also stimulate intervention use. It has been argued that self-regulatory competence plays a vital role in adolescents' DI (de Vet \& de Ridder, 2015; DeSmet et al., 2014; King et al., 2014; Kirwan, Duncan, Vandelanotte, \& Mummery, 2012; Lustria, Cortese, Noar, \& Glueckauf, 2009; Middelweerd et al., 2015). By implementing additional strategies such as higher interactivity and visual attractiveness, which are inherent aspects of serious gaming (Crutzen et al., 2008; Crutzen et al., 2011; DeSmet et al., 2014), we aimed to overcome low reach and low intervention adoption rates. We made "Balance It" free of charge to secondary vocational education students enrolled in the "Lifestyle Reality Check" system (see Spook, Paulussen, Kok, \& van Empelen, 2015), to take into account the socioeconomic disadvantage of our specific target population (a factor which is frequently overlooked, see Jane, Foster, Hagger, \& Pal, 2015).

The main findings of the "Balance It" intervention showed short-term effects on secondary vocational education students' DI and PA. After four weeks, students in the intervention group reported a significantly stronger decrease in soft drink consumption as compared to the control group, and an increase in active transport (whereas the control group reported decreased use of active transport). No significant differences in change scores were found for fruit and vegetable consumption, snack consumption, and exercise between the two groups. However, only $28 \%$ of the intervention group reported that they had played "Balance It". Therefore, a second comparison was made between "intervention users" (i.e., this $28 \%$ of the intervention group) and "non-users" (i.e., the remaining $72 \%$ of the intervention group $+100 \%$ of the control group). 
Although there are several limitations inherent in the second comparison of "intervention users" and "non-users" (e.g., limited power and validity due to a small number of intervention users and noise in the joined "non-users" group, respectively), "intervention users" showed an increase of .51 pieces of fruit on average, as well as increased vegetable consumption (+ .06 portions a day on average), and decreased snack consumption (-.20 portions a day on average). A small increase in soft drink consumption was only marginally significant, and any increases in active transport and PA were not significant. This is consistent with findings from Baranowski et al. who showed that playing serious games such as "Diab" and "Nano" increased fruit and vegetable intake, but did not improve the level of PA in young people (Baranowski et al., 2011). However, it is worth noting that any effects of "Balance It" are assessed by examining change scores over a four-week period. Yet students may have set their PA goals at a weekly level (e.g., to exercise twice a week), meaning that this type of game play would take six weeks to complete. As such, post-test measures already took place prior to finishing PA-related games, which may explain the lack of direct effects of "Balance It" on PA. However, we did find a significant increase in PA intentions among "intervention users", which may lead to effects on PA at a later time (Spook et al., submitted for publication).

Overall, we can conclude that, despite the lack of long term effects, "Balance It" has the potential to change secondary vocational education students' dietary intake and physical activity level. However, as with $\mathrm{mEMA}$, it appears to be hampered by limited compliance. It is important to note that we have applied prompting techniques to stimulate the use of "Balance It" (i.e., intervention compliance), a technique that has frequently been recommended as a means of stimulating behavior change (Abraham \& Michie, 2008; Dusseldorp, van Genugten, van Buuren, Verheijden, \& van Empelen, 2013; Fogg, 2009; Kok et al., 2015; Webb et al., 2010). Yet despite its potential, the selfprompting feature in "Balance It" was barely used. Possibly, these prompts were seen as disruptive, which, according to Bort-Roig et al. (Bort-Roig, Gilson, Puig-Ribera, Contreras, \& Trost, 2014), can limit engagement in health behavior. As with the monitoring burden that may be experienced in $\mathrm{mEMA}$ research, similar trade-offs between prompt burden and forgetting to play may need to be further assessed.

Moreover, trade-offs may also be necessary to balance health promotion objectives with game usability objectives or game design, as compatibility issues between the two may arise during the developmental process behind serious games. For example, during the development of "Balance It", 
multiple focus groups were conducted with the aim of optimally adjusting the intervention to our target population. As part of this process, the behavior change experts recommended that a peer support system was put in place, based on participants' expressed need to share their progression with their peers and to provide and receive support, and backed up by Theories of Social Networks and Social Support (Bartholomew, Parcel, Kok, Gottlieb, \& Fernández, 2011; Heaney \& Israel, 2008), However, game experts also stressed the importance of the limited number of layers within the mobile game environment, as this may disrupt game flow. As such, we compromised by creating an additional "Balance It" website, providing information and examples of implementation intentions, an overview of their overall progression, and a peer support system. However, despite this additional investment, the process evaluation of "Balance It" showed that the use of this website and its peer support system was rather disappointing.

There are many other researchers who have faced problems with the use of peer-support systems (Binks \& van Mierlo, 2010; Eysenbach, Powell, Englesakis, Rizo, \& Stern, 2004; McConnon, Krik, \& Ransley, 2009). McConnon et. al. (2009) have indicated that the least utilized and appreciated part of their online intervention were the social support features. Similarly, Binks and van Mierlo (2010) reported low usage $(7.8 \%)$ of the social support message board implemented within their internet weight loss program. However, social support has shown to be a potentially beneficial component of online weight loss programs (Greaves et al., 2011; Jeffery et al., 1984; Kelsey, Earp, \& Kirkley, 1997; Turner-McGrievy \& Tate, 2013). A quick comparison of "Balance It" and the online intervention developed by Turner-McGrievy and Tate (2013), who reported positive associations between Twitter use and weight loss, showed that Turner-McGrievy and Tate had a study weight loss counselor posting two Tweets a day to stimulate a discussion among study participants, and encouraged participants to engage with Twitter during the program (Turner-McGrievy \& Tate, 2013). Although it is not clear if these two components explain social network use, it may be interesting fruitful to evaluate and perhaps incorporate this strategy in a renewed "Balance It" program.

Finally, a few more challenges that are related to serious gaming interventions should be addressed. As recently indicated by DeSmet et al. (2014), intervention effects are likely to decrease over time (DeSmet et al., 2014), suggesting that the effects that we found at posttest, four weeks after initial intervention use, may diminish or disappear at follow-up. Unfortunately, the response rate at follow-up was too low to be included in the "Balance It" evaluation. This lack of response may be due 
to the fact that participants were approached via email to fill in a lengthy questionnaire instead of face-to-face as during pre- and posttest measures. Therefore, the length of the questionnaire and the presence of a research assistant during the follow-up measure may be important considerations to bear in mind for future evaluation studies.

\section{PART III Promises and Pitfalls of Applying IM}

To date, multiple serious games have been developed to target health behavior (Wouters, van der Spek, \& van Oostendorp, 2009). Although they are assumed to increase e-Health intervention effectiveness, effect sizes have been limited and comparable to computer-tailored interventions (DeSmet et al., 2014). One possible explanation for these disappointing results may be found in the lack of systematic development underlying these serious games (DeSmet et al., 2014). As addressed by Mohr, Schueller, Montague, Burns, and Rashidi (2014) and Crutzen (2014), technical behavioral interventions could be optimized if they would be developed systematically (e.g., according to Intervention Mapping; IM), and in close collaboration with individuals with different areas of expertise (Crutzen, 2014; Mohr, Schueller, Montague, Burns, \& Rashidi, 2014). These recommendations were taken into account during the development of the "Balance It" intervention.

Although the IM protocol provides a strong foundation for the intervention because of its systematic theory- and evidence-based approach (Bartholomew et al., 2011), nevertheless, some challenges arose when applying IM to serious gaming interventions. Normally, design protocols based on IM describe the six steps (i.e., 1) a needs assessment, 2) matrices of objectives, 3) methods and applications, 4) intervention program development, 5) intervention implementation, and 6) evaluation) in chronological order and emphasize the importance of iterations between these steps (Bartholomew et al., 2011). In terms of serious gaming interventions, this theory and evidencebased foundation is grounded in both health promotion and gaming theory (DeSmet et al., 2014). In addition to the significant increase in matrices of objectives, methods and applications, and increased difficulty in terms of the intervention program development, it was also challenging to describe the interrelatedness of health promotion and the usability of the gaming program within the current IM framework.

A second challenge we encountered when applying IM to serious game design was the dual level implementation required. First, we needed an existing system (or context) in which to implement 
"Balance It", increasing the reach of our intervention (cf. Crutzen et al., 2013). This was conceptualized as the contextual implementation level. However, in order to encourage the actual use of "Balance It", the intervention had to be implemented within the daily lives of our participants, requiring an individual implementation level. As such, there were multiple considerations to be taken into account, both at a theoretical and an implementation level. To overcome these challenges, we conceptually altered the IM framework, emphasizing the equal importance of both behavior change processes and intervention use in IM steps 2 and 3, and the two levels of implementation required in IM steps 2 and 3, and 5 (see Figure 1; Spook et al., 2015). However, this was the first time that these alterations to the framework were made, so future research on the usability of the adapted framework is highly recommended.

Figure 1: Design Rationale of a Serious Game1

\begin{tabular}{|c|c|c|c|}
\hline & IM Step & Health Promotion Goal & Usability Goal \\
\hline \multirow{9}{*}{$\longrightarrow$} & 1 & \multicolumn{2}{|c|}{ Needs Assessment } \\
\hline & & \multicolumn{2}{|c|}{ Matrices } \\
\hline & 2 & $\begin{array}{l}\text { Performance Objectives - Health } \\
\text { Determinants - Health } \\
\text { Change Objectives - Health }\end{array}$ & $\begin{array}{c}\text { Performance Objectives - Usability } \\
\text { Determinants - Usability } \\
\text { Change Objectives - Usability }\end{array}$ \\
\hline & \multirow{2}{*}{3} & \multicolumn{2}{|c|}{ Methods \& Practical Applications } \\
\hline & & Behavior Change Techniques & Persuasive Techniques \\
\hline & \multirow[b]{2}{*}{4} & \multicolumn{2}{|c|}{ Concept Intervention Program } \\
\hline & & \multicolumn{2}{|c|}{$\begin{array}{c}\text { Game Genre \& Concept } \\
\text { Game Elements } \\
\text { Game Process } \\
\text { Concept and Usability Testing }\end{array}$} \\
\hline & 5 & \multicolumn{2}{|c|}{ Program Adoption and Implementation Plan } \\
\hline & 6 & \multicolumn{2}{|c|}{ Evaluation Plan } \\
\hline
\end{tabular}

${ }^{1}$ derived from (Spook et al., 2015)

\section{PART IV}

This last part of the General Discussion describes characteristics of the targeted population (i.e., secondary vocational education students), practical constraints of mHealth research, implications for research and practice, and future research directions. Finally, we sum up this dissertation with a General Conclusion. 


\subsection{Targeted Population: Secondary Vocational Education Students}

As outlined in the previous paragraphs, compliance with "mEMA" and "Balance It" was not optimal. Although disappointing compliance rates are prevalent among different demographic groups, it should be noted that we targeted a difficult to reach group of socioeconomically disadvantaged youth, in which overweight is highly prevalent (Bernaards \& Buuren van, 2012; Schönbeck \& van Buuren, 2010), and who often participate in secondary vocational education (Bernaards \& Buuren van, 2012; Ministery of Education, Culture, and Science, 2012). Students from this demographic group are assumed to be easier to reach in terms of mHealth research, as it is highly common for these students to possess smartphones and use them on a daily basis (Spook et al., 2013). Therefore mHealth assessment and intervention studies are promising ways of reaching secondary vocational education students.

To enhance the likelihood of use of "mEMA" and "Balance It", we conducted formative research with these students to account for user experiences (Crutzen et al., 2013). Students were observed during alpha- and beta-testing of the "mEMA" and "Balance It" apps (i.e., test versions of the apps). Acceptability, usability, and game dynamics were discussed during multiple focus groups (see Spook et al., 2015). Formative research is a recommended strategy in mHealth research (DeSmet, 2015). DeSmet et al. found support for the effectiveness of game design in which participants had informant roles (as compared to co-design roles) (DeSmet, 2015). In terms of "mEMA", our formative research resulted in a reduced number of prompt signals used, in an attempt to limit monitoring burden (see Spook et al., 2013). Formative research conducted to optimize "Balance It" resulted in an additional peer-support system being put in place as well as an additional game level structure. Although both components were evaluated in a moderately positive way, the use of the "Balance It" app and website were still rather disappointing.

\subsection{Practical Constraints of mHealth Research}

Besides methodological issues that were associated with "mEMA" or "Balance It" in particular, that have already been raised in the relevant chapters, we also experienced some practical constraints while carrying out the two studies. First, due to circumstances (i.e., one of the technical developers was declared bankrupt during the data collection of "mEMA-PA") and time pressure, we were forced to allow higher professional education students to participate in the study to gain at least some understanding of factors associated with real-time PA. It should also be noted that recruiting secondary 
vocational education students is becoming increasingly difficult (e.g., Simons, 2015), even though it has previously been stressed that students are easy to reach via the school systems (Crutzen et al., 2013). In terms of our own data collection, school coordinators were, in general, very interested in our study, but mentioned their lack of time, and/or the overload of requests by Universities and Higher Professional Education institutions as important reasons for not participating in one of the studies presented in this thesis.

When approaching students through school systems, it is important to try and avoid contamination effects in intervention studies (Donner \& Klar, 2000; McKenzie, Ryan, Di Tanna, \& Cochrane Consumers and Communication Review Group, 2014), by carrying out a Group-Randomized Trial (GRT). GRTs evaluate intervention effects at a group level (Murray, Varnell, \& Blitstein, 2004). Additionally, the school coordinators involved in our studies preferred their students to be in the same condition (i.e., intervention group or control group). When the allocation of identifiable groups is necessary, GRT can be seen as "the gold standard" (Murray et al., 2004). However, it should be noted that for the evaluation of "Balance It" in which we also compared the "intervention users" from the intervention group with "non-users", contamination effects may have influenced the results after all. As the students allocated to the group of "non-users" are derived from both the intervention group and the control group, the clarity of the non-users group was diminished (i.e., because part of the "non-users" group were exposed to the intervention). Nevertheless, this comparison between "intervention users" and "non-users" was undertaken as part of a pilot study. We recommend that a main GRT, comparing an intervention group with a control group, is carried out in future research.

Other minor drawbacks in mHealth research include: 1) the differences between operating systems and versions of smartphones, resulting in programming constraints (Kuntsche \& Labhart, 2013; Spook et al., 2013), 2) the limited screen size of smartphones requiring practical solutions (e.g., VAS sliders, see (Spook et al., 2013), 3) the need for a limited number of mEMA items or layers within mobile interventions to reduce the likelihood of drop-out due to experienced burden, and 4) the fact that smartphones may be less commonly used and appreciated among specific target populations (e.g., the elderly; Kuntsche \& Labhart, 2013; Kurniawan, 2008), hampering mHealth use and effectiveness. However, mHealth interventions may well be worth the investment because of their potential impact on societal health via increased and sustained effectiveness and use. Still, more evidence-based research on mHealth tools and their cost-effectiveness is to be highly encouraged (see van Heerden, 
Tomlinson, \& Swartz, 2012). Lastly, Kuntsche and Labhart (2013) mention the researchers' creativity and the programmers' skills as potential drawbacks (Kuntsche \& Labhart, 2013), but these drawbacks might also be considered to be strengths, especially when the two groups of experts collaborate efficiently.

\subsection{Implications for Research and Practice}

Based on the study findings and experiences from the developmental processes of "mEMA" and "Balance It", we have made several recommendations. First, in order to create a strong theoretical and evidence-based foundation for serious games, we recommended the systematic development of this type of intervention (e.g., using the Intervention Mapping (IM) protocol; Bartholomew et al., 2011). As such, a dual process which targets health promotion and gaming simultaneously, as well as applying implementation strategies at both the individual level (e.g., usability objectives in IM steps 2 and 3), and the contextual level (e.g., implementation objectives in IM step 5) is highly recommended.

Second, trade-offs have to be made when collaborating with professional designers (or other parties). When all parties stand their ground in terms of the techniques they want to apply within the game, conflicts may arise. Therefore, it is important to respect each other's expertise, to clearly outline the methods that should be implemented under which circumstances (i.e., the parameters for use; Bartholomew et al., 2011), to take minutes of meetings in order to confirm mutual understanding, and to keep communicating about the progress.

\subsection{Directions for Future Research}

Based on the findings and experiences from the developmental processes of " $m E M A$ " and "Balance It", several questions have come up that may give direction to future research. First, "mEMA" was originally developed as a multicomponent smartphone app, gathering real-time survey data (i.e., the short questionnaires in the "mEMA" app), GPS data, and data derived from a built-in accelerometer. Because of the wide scope of the studies included in the present thesis, we did not manage to validate the accelerometer data and to perform a hybrid study comparing the different types of data. Nevertheless, mEMA research is designed to increase the accuracy of measuring complex behaviors and to reduce the recall biases inherent in survey designs. It would therefore be preferable to compare the different types of data (i.e., regular survey data, mEMA questionnaires, and objective measures) within a single study. As such, we may gain a more accurate understanding of the benefits 
of $\mathrm{mEMA}$ and the data would provide a more comprehensive picture of the complex health behavior being studied.

One of the other main challenges of mEMA research is non-compliance. As described in Chapter 2, possible explanations for non-compliance may be found in monitoring burden, or "monitoring boredom", as participants in the pilot study reported experienced use of the "mEMA" app as boring (Spook et al., 2013), because they were prompted multiple times a day for several consecutive days with the same questions. It may, therefore, be interesting to further investigate factors that influence compliance with mEMA research, and to study the degree of monitoring boredom experienced among different populations.

Finally, further research on the use of serious games is needed to increase engagement with the intervention. Recently, some interesting studies have been designed to increase exposure to interventions, as well as increase their use. These studies focus on the implementation of meta-interventions (Albarracín, Durantini, Earl, Gunnoe, \& Leeper, 2008) or novellas (Baranowski \& Baranowski, 2015). Albarracin (2008) has reported that intervention introductions, or meta-interventions, which challenge participants' resistance to change can increase participation rates (Albarracín et al., 2008). Within the land of gaming, Baranowski and Baranowski (2015) are developing such a meta-intervention in the form of a "novella" (Baranowski \& Baranowski, 2015). Previous video games have often relied on narratives to provide situational context and modeling of behavior change, but storytelling can interrupt game immersion and alienate players (Baranowski \& Baranowski, 2015). This finding is supported by a review published by DeSmet et al., which showed no significant associations between (in-game) narratives and game effectiveness (DeSmet, 2015). Therefore, Baranowski and Baranowski (2015) suggest that it may be more effective to deliver these stories outside of gameplay, and in line with this, their first pilot showed an increased interest in gameplay after participants had read the novella. This study shows promising results and it may be worthwhile exploring whether novellas will also be effective in terms of "Balance It". 


\section{General Conclusion}

Overall, we can conclude that with the development of "mEMA" and "Balance It" we gained a better understanding of the dynamics in dietary intake and physical activity in students' daily life and made a good attempt to change these complex behaviors among a population that is difficult to target. The two smartphone applications were both perceived to be feasible and usable tools, as the majority of these students carried their smartphone around with them every day and both the applications were rated moderately positively. Although several limitations have been addressed and directions for future research have been recommended in the General Discussion, we have been fully transparent in how we applied the Intervention Mapping protocol during the developmental process of a serious game intervention, and, as such, we have contributed to the development of e- and mHealth research in general. 


\section{References}

Abraham, C., \& Michie, S. (2008). A taxonomy of behavior change techniques used in interventions. Health Psychology, 27(3), 379-387.

Ajzen, I. (1991). The theory of planned behavior. Organizational Behavior and Human Decision Processes, 50, 179-211.

Albarracín, D., Durantini, M. R., Earl, A., Gunnoe, J. B., \& Leeper, J. (2008). Beyond the most willing audiences: A meta-intervention to increase exposure to HIV-prevention programs by vulnerable populations. Health Psychology, 27, 638-644.

Baranowski, T., \& Baranowski, J. (2015). Using fictional novellas as narrative companion for vegetable parenting game. Proceedings of the International Society of Nutritional Behavior and Physical Activity: Advancing Behavior Change Science. Edinburgh.

Baranowski, T., Baranowski, J., Thompson, D., Buday, R., Jago, R., Juliano Griffith, M., ... Watson, K. B. (2011). Video game play, child diet, and physical activity behavior change: A randomized clinical trial. American Journal of Preventive Medicine, 40(1), 33-38.

Bartholomew, L. K., Parcel, G. S., Kok, G., Gottlieb, N. H., \& Fernández, M. E. (Eds.). (2011). Planning health promotion programs: An intervention mapping approach. San Francisco: Jossey-Bass.

Bernaards, C., \& Buuren van, S. (2012). Rapportage veranderingen in het beweeggedrag van mbo studenten. (No. R10185). Leiden, the Netherlands: TNO Behavioural and Societal Sciences.

Binks, M., \& van Mierlo, T. (2010). Utalization patterns and user characteristics of an ad libitum internet weight loss program. Journal of Medical Internet Research, 12(1), e9.

Bort-Roig, J., Gilson, N. D., Puig-Ribera, A., Contreras, R. S., \& Trost, S. G. (2014). Measuring and influencing physical activity with smartphone technology: A systematic review. Sports Medicine, 44, 671-686.

Broderick, J. E., Schwartz, J. E., Shiffman, S., Hufford, M. R., \& Stone, A. A. (2003). Signaling does not adequately improve diary compliance. Annals of Behavioral Medicine, 26, 139-148. 
Conner, M., McEachan, R., Jackson, C., McMillan, B., Woolridge, M., \& Lawton, R. (2013). Moderating effect of socioeconomic status on the relationship between health cognitions and behaviors. Annals Behavioral Medicine, 46, '19-30.

Crutzen, R. (2014). The behavioral intervention technology model and intervention mapping: The best of both worlds. Journal of Medical Internet Research, 16(8), e188.

Crutzen, R., de Nooijer, J., Brouwer, W., Oenema, A., Brug, J., \& de Vries, N. K. (2008). Internet-delivered interventions aimed at adolescents: A delphi study on dissemination and exposure. Health Education Research, 23(3), 427-439.

Crutzen, R., de Nooijer, J., Brouwer, W., Oenema, A., Brug, J., \& de Vries, N. K. (2011). Strategies to facilitate exposure to internet-delivered health behavior change interventions aimed at adolescents or young adults: A systematic review. Health Education Behaviour, 38(1), 49-62.

Crutzen, R., Kohl, L., \& de Vries, N. K. (2013). Kennissynthese online preventie

DeSmet, A. (2015). Understanding adolescent bystander behavior in cyberbulling and the potential of serious digital games to promote prosocial behavior and other healthy lifestyles.[Dissertation]. Ghent: Ghent University.

DeSmet, A., van Ryckeghem, D., Compernolle, S., Baranowski, T., Thompson, D., Crombez, G., ... de Bourdeaudhuij, I. (2014). A meta-analysis of serious digital games for healthy lifestyle promotion. Preventive Medicine, 69, 95-107.

Donner, A., \& Klar, N. (2000). Design and analysis of cluster randomization trials in health research. London: Arnold Publishing.

Douketis, J. D., Maci, C., Thabane, L., \& Williamson, D. F. (2005). Systematic review of long-term weight loss studies in obese adults: Clinical significance and applicability to clinical practice. International Journal of Obesity, 29, 1153-1167.

Dusseldorp, E., van Genugten, L., van Buuren, S., Verheijden, M. W., \& van Empelen, P. (2013). Combinations of techniques that effectively change health behavior: Evidence from meta-analysis. Health Psychology, 
Eisenberg, M. E., Neumark-Sztainer, D., Story, M., \& Perry, C. (2005). The role of social norms and friends' influences on unhealthy weight-control behaviors among adolescent girls. Social Science \& Medicine, 60(6), 1165-1173.

Eysenbach, G., Powell, J., Englesakis, M., Rizo, C., \& Stern, A. (2004). Health related virtual communities and electronic support groups: Systematic review of the effects of online peer to peer interactions. Britisch Medical Journal, 328, 1166.

Fogg, B. J. (2009). A behavior model for persuasive design. Proceedings of the 4th International Conference on Persuasive Technology, ACM.

Greaves, C. J., Sheppard, K. E., Abraham, C., Hardeman, W., Roden, M., Evans, P. H., ... The IMAGE Study Group. (2011). Systematic review of reviews of intervention components associated with increased effectiveness in dietary and physical activity interventions. BMC Public Health, 11, 119.

Haedt-Matt, A. A., \& Keel, P. K. (2011). Revisiting the affect regulation model of binge eating: A meta-analysis of studies using ecological momentary assessment. Psychological Bulletin, 137(4), 660-681.

Hagger, M. S., Wood, C., Stiff, C., \& Chatzisarantis, N. L. (2010). Ego depletion and the strength model of self-control: A meta-analysis. Psychological Bulletin, 136(4), 495.

Hardcastle, S. J., Hancox, J., Hattar, A., Maxwell-Smith, C., Thogersen-Ntoumani, C., \& Hagger, M. S. (2015). Motivating the unmotivated: How can health behavior be changed in those unwilling to change? Frontiers in Psychology, 6, 1-4.

Heaney, C. A., \& Israel, B. A. (2008). Social networks and social support. In K. Glanz, B. K. Rimer \& K. Viswanath (Eds.), Health behavior and health education: Theory, research, and practice. (4th ed., pp. 189-210). San Francisco: Jossey-Bass.

Heron, K. E., \& Smyth, J. M. (2010). Ecological momentary interventions: Incorporating mobile technology into psychosocial and healthb ehaviour treatments. British Journal of Health Psychology, 1, 1-39.

Hufford, M. R., \& Shiffman, S. (2003). Subject-reported outcomes: Assessment methods. Disease Management and Health Outcomes, 11, 77-86. 
Jane, M., Foster, J., Hagger, M., \& Pal, S. (2015). Using new technologies to promote weight management: A randomised controlled trial study protocol. BMC Public Health, 15(1), 509-518.

Jeffery, R. W., Bjornson-Benson, W. M., Rosenthal, B. S., Lindquist, R. A., Kurth, C. L., \& Johnson, S. L. (1984). Correlates of weight loss and its maintenance over two years of follow-up among middle-aged men. Preventive Medicine, 13(2), 155-168.

Kelsey, K., Earp, J. A. L., \& Kirkley, B. G. (1997). Is social support beneficial for dietary change? A review of the literature. Family \& Community Health, 20(3), 70-82.

Kok, G., Gottlieb, N. H., Peters, G. Y., Mullen, P. D., Parcel, G. S., Ruiter, R. A., . . Bartholomew, L. K. (2015). A taxonomy of behavior change methods; an intervention mapping approach. Health Psychology Review, (just-accepted), 1-32.

Krebs, P., Prochaska, J. O., \& Rossi, J. S. (2010). A meta-analysis of computer-tailored interventions for health behavior change. Preventive Medicine, 51, 214-221.

Kremers, S. P. J., de Bruin, G., Visscher, T. L. S., van Mechelen, W., de Vries, N., \& Brug, J. (2006). Environmental influences on energy balance-related behaviors: A dual-process view. International Journal of Behavioral Nutrition and Physical Activity, 3(9).

Kroeze, W., Werkman, A., \& Brug, J. (2006). A systematic review of randomized trials on the effectiveness of computer-tailored education on physical activity and dietary behaviors. Annals of Behavioral Medicine, 31(3), 205-223.

Kuntsche, E., \& Labhart, F. (2013). Using personal cell phones for ecological momentary assessment. European Psychologist, 18(1), 3-11.

Kurniawan, S. (2008). Older people and mobile phones: A multi-method investigation. International Journal of Human-Computer Studies, 66(12), 889-901.

Lobstein, T., Baur, L., \& Uauy, R. (2004). Obesity in children and young people: A crisis in public health. Obesity Reviews, 5, 4-85.

Marszalek, J., Morgulex-Adamowicz, N., Rutkowska, I., \& Kosmol, A. (2014). Using ecological momentary assessment to evaluate current physical activity. BioMed Research International, , 1-9. 
McConnon, A., Krik, S. F., \& Ransley, J. K. (2009). Process evaluation of an internet-based resource for weight control: Use and views of an obese sample. Journal of Nutrition Education and Behavior., 41(4), 261-267.

McEachan, R. R. C., Conner, M., Taylor, N., \& Lawton, R. J. (2011). Prospective prediction of health-related behaviors with the theory of planned behavior: A meta-analysis. Health Psychology Review, 5, 97-144.

McKenzie, J., Ryan, R., Di Tanna, G. L., \& Cochrane Consumers and Communication Review Group. (2014). Cochrane consumers and communication review group: Cluster randomised controlled trials. (). http://cccrg.cochrane.org/sites/cccrg.cochrane.org/files/uploads/ClusterRCTs.pdf: Cochrane Consumers and Communication Review Group.

Ministery of Education, Culture, and Science. (2012). Kerncijfers 2007-2011. (No. 9879059101562). Kelpen-Oler: Hub Tonnaer B.V.

Mohr, D. C., Schueller, S. M., Montague, E., Burns, M. N., \& Rashidi, P. (2014). The bheavioral intervention technology model: An integrated conceptual and technological framework for eHealth and mHealth interventions. Journal of Medical Internet Research, 16(6), e146.

Motl, R. W., Dishman, R. K., Saunders, R., Dowda, M., Felton, G., Ward, D. S., \& Pate, R. R. (2002). Examining social-cognitive determinants of intention and physical activity among black and white adolescent girls using structural equation modeling. Health Psychology, 21, 459-467.

Murray, D. M., Varnell, S. P., \& Blitstein, J. L. (2004). Design and analysis of group-randomized trials: A review of recent methodological developments. American Journal of Public Health, 94(3), 423-432.

Rhodes, R. E., \& de Bruijn, G. J. (2013). How big is the physical activity intention-behaviour gap? A meta-analysis using the action control framework. British Journal of Health Psychology, 18, 296-309.

Ronda, G., van Assema, P., \& Brug, J. (2001). Stages of change, psychological factors and awareness of physical activity levels in the netherlands. Health Promotion International, 16(4), 305-314.

Sallis, J. F., Johnson, M., Calfas, K., Caparosa, S., \& Nichols, J. (1997). Assessing the perceived physical environmental variables that may influence physical activity. Research Quarterly for Exercise and Sport, 68, 345-351. 
Salvy, S., Jarrin, D., Paluch, R., Irfan, N., \& Pliner, P. (2007). Effects of social influence on eating in couples, friends and strangers. Appetite, 49(1), 92-99.

Schönbeck, Y., \& van Buuren, S. (2010). Resultaten vijfde landelijke groeistudie. (factsheet). Leiden: TNO.

Simons, M. (2015). Active video games: Can they contribute to the prevention of excessive weight gain in gaming adolescents?

Sokolovsky, A. W., Mermelstein, R. J., \& Hedeker, D. (2014). Factors predicting compliance to ecological momentary assessment among adolescent smokers. Nicotine \& Tobacco Research, 16(3), 351-358.

Spence, J. C., \& Lee, R. E. (2003). Toward a comprehensive model of physical activity. Psychology of Sport and Exercise, 4, 7-24.

Spook, J. E., Paulussen, T., Kok, G., \& van Empelen, P. (2013). Monitoring dietary intake and physical activity electronically: Feasibility, usability, and sensitivity of a mobile-based ecological momentary assessment (mEMA) tool. Journal of Medical Internet Research, 15(9), e214.

Spook, J. E., Paulussen, T., Kok, G., \& van Empelen, P. (2015). Design rationale behind the serious self-regulation game intervention "balance it": Overweight prevention among secondary vocational education students in the Netherlands. Games for Health Journal: Research, Development, and Clinical Applications, 4(6).

Spook, J. E., Paulussen, T., Kok, G., \& van Empelen, P., submitted for publication. Factors determining physical activity of emerging young adults: A mobile-based Ecological Momentary Assessment (mEMA) study.

Spook, J.E., Paulussen, T., Kok, G., van Empelen, P., submitted for publication. The role of motivational and contextual factors on intentional and craving-induced snack consumption: A mobile-based Ecological Momentary Assessment study.

Stone, A. A., Broderick, J. E., Schwarz, J. E., Shiffman, S., Litcher-Kelly, L., \& Calvanese, P. (2003). Intensive momentary reporting of pain with an electronic diary: Reactivity, compliance, and patient satisfaction. Pain, 104, 343-351. 
Stone, A. A., Shiffman, S., Atienza, A. A., \& Nebeling, L. (2007). The science of real-time data capture. self-reports in health research. New York: Oxford University Press.

Stone, A. A., Shiffman, S., \& de Vries, M. W. (2000). Ecological momentary assessment. In D. Kahneman, E. Diener \& N. Schwarz (Eds.), Well-being: The foundations of hedonic psychology. (pp. 26-39). New York: Russell Sage Foundation.

Stone, A. A., Shiffman, S., Schwartz, J. E., Broderick, J. E., \& Hufford, M. R. (2003). Patient compliance with paper and electronic diaries. Controlled Clinical Trials, 24(2), 182-199.

Stone, A., \& Shiffman, S. (2002). Capturing momentary self-report data: A proposal for reporting guidelines. Annals of Behavioral Medicine, 24, 236-243.

SURFnet. (2013). Trendonderzoek mobiele technologie: Trends gebruik mobiele technologie binnen het hoger onderwijs en onderzoek. (No. G5759).SURFnet.

Turner-McGrievy, G. M., \& Tate, D. F. (2013). Weight loss social support in 140 characters or less: Use of an online social network in a remotely delivered weight loss intervention. Translational Behavioral Medicine, 3(3), 287-294.

Van Genugten, L. (2011). Prevention of weight gain among adults: Development and evaluation of a computer-tailored self-regulation intervention.

Van Heerden, A., Tomlinson, M., \& Swartz, L. (2012). Point of care in your pocket: A research agenda for the field of m-health. Bulletin of the World Health Organization, 90, 393-394.

Verweij, L. M., Coffeng, J., van Mechelen, W., \& Proper, K. I. (2011). Meta-analyses of workplace physical activity and dietary behaviour interventions on weight outcomes. Obesity Reviews, 12, 406-429.

Webb, T., Joseph, J., Yardley, L., \& Michie, S. (2010). Using the internet to promote health behavior change: A systematic review and meta-analysis of the impact of theoretical basis, use of behavior change techniques, and mode of delivery on efficacy. Journal of Medical Internet Research, 17(12), e4.

Wouters, P., van der Spek, E. D., \& van Oostendorp, H. (2009). Current practices in serious game research: A review from a learning outcomes perspective. Games-Based Learning Advancements for Multisensory Human Computer Interfaces: Techniques and Effective Practices, , 232-255. 

Valorisation Addendum

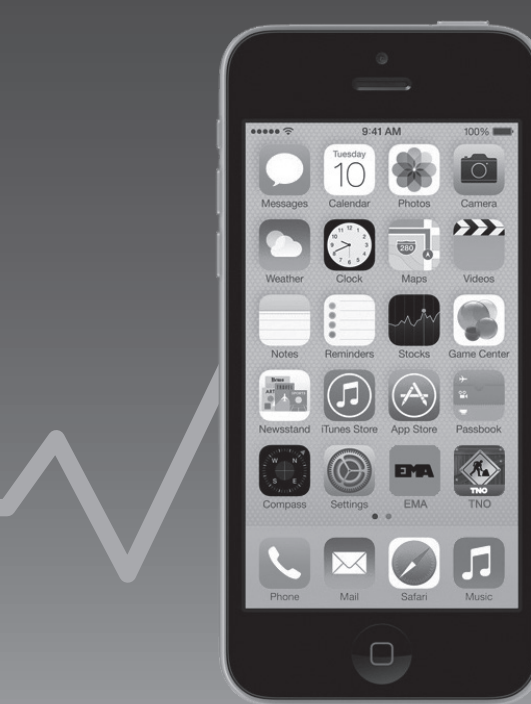




\section{Valorisation Addendum}

One area of the "MBO Raad" (the Netherlands Association of Vocational Education and Training Colleges) and the "Lifestyle Reality Check" foundation's concern is the common and cumulating prevalence of overweight among secondary vocational education students in the Netherlands. Although the "Lifestyle Reality Check's student tracking system" allows the foundation to monitor health-related behaviors among secondary vocational education students, extension of the current system to facilitate behavior change is desirable. As the findings of this dissertation "Keeping it real: Understanding and changing health behavior in daily life" provide meaningful insights into overweight-related behaviors among those students, these findings may be of great interest to the "MBO Raad" and the "Lifestyle Reality Check" foundation and, of course, students' health in general.

As explained in Chapter 2, students are well targeted via mobile devices. They carry their smartphones around at all times, providing a 24/7 platform for real-time monitoring and intervention applications. Although mobile-based Ecological Momentary Assessment (mEMA) and Interventions (mEMI) are options for the "MBO Raad", the "Lifestyle Reality Check" foundation, researchers and relevant industries to target health-related behaviors among students, they should keep in mind that their application should be tailored to the target populations' needs and wants, optimizing overall use of the application and preventing interference with students' overall wellbeing.

The outlined mEMA studies in Chapters 3 and 4 emphasize the complexity of students' dietary intake and physical activity, in terms of determinants and how they change over time. The type of data derived from mEMA studies provide more accurate insights into health behaviors compared to selfreported cross-sectional data, as several biases (e.g., recall bias) are diminished by real-time, realworld monitoring. The "mEMA app" that has been developed may be of interest to other behavioral and health scientists to measure complex behaviors over time.

The "Balance It app" to change health-related behaviors in a gamified fashion may especially in the interest of the secondary vocational education students, the "MBO Raad" and the "Lifestyle Reality Check" foundation. The "Balance It app" is currently free of charge available in the Google and ioS appstores allowing students to play the game. However, the game may be more successful when embedded within an existing system, such as the "Lifestyle Reality Check system". As an extension of the current "Lifestyle Reality Check system" is desirable to facilitate health behavior change, 
a linkage between the system and "Balance It" may be of interest. TNO, the "MBO Raad" and the "Lifestyle Reality Check" foundation are already in dialogue about merging the two systems.

While the "mEMA app" and "Balance It app" are innovative in itself, applying Intervention Mapping (IM) to the development of an mHealth intervention and using a dual theoretical foundation in both health promotion and app use/gaming also enhances the innovative character of the present dissertation. To share our new knowledge with society, this dissertation will be made available online. Hardcopies will also be distributed among academics, the "MBO Raad" and the "Lifestyle Reality Check" foundation, contact persons at the participating secondary vocational education schools in the Netherlands, interested professional game developers, and everyone else who is interested in the research described in this dissertation: “Keeping it real: Understanding and changing health behavior in daily life". 

Summary 


\section{General Introduction}

With the increasing numbers of overweight and obese individuals in developed countries, not only the physical appearance of people gets affected, but it can also lead to serious physical and mental health problems. Being overweight has, therefore, been recognized as a serious disease. Health problems that are associated with excess weight include for example type 2 diabetes mellitus and cardiovascular disease. These diseases, consequently, drive up healthcare costs. As the prevalence of overweight and obesity is most evident in low socio-economic status (SES) families and minority groups, it is important to focus intervention efforts on this group. More specifically, targeting youth from low-SES families is vital as being overweight during childhood is a strong predictor of being overweight during adulthood. As many low SES youth participate in secondary vocational education (Dutch "Middelbaar Beroeps Opleiding", or MBO), we mainly focused on these students in the studies presented in this dissertation.

To understand and change overweight-related behaviors (ORBs) of secondary vocational education students' in daily life, we used the Intervention Mapping (IM) protocol. The Intervention Mapping (IM) protocol describes the six steps involved in systematically planning a theory- and evidence-based intervention. The protocol starts with a needs assessment (step 1), in which the health problem, the behavioral and environmental causes of this problem, and any determinants of these causes are assessed. In step 2 of IM, the change objectives for the intervention are specified. Change objectives specify what needs to be changed with regard to a specific determinant in order to be able to perform the desired behavior (i.e., the performance objectives). In step 3, theory- and evidencebased behavior change methods or techniques are translated into practical applications. Notably, these methods refer to the theoretical techniques by which behavior (or determinants of behavior) is (or are) expected to change. Importantly, the theory provides the requirements that need to be met for the effective use of a technique. Step 4 concerns the logical integration of the previously selected applications into a coherent intervention program design. In step 5, implementation objectives are formulated to ensure effective program use, and finally, in step 6, an evaluation plan is conducted in order to validate the intervention.

In line with the IM protocol, the present dissertation can be divided into two parts: 1) the needs assessment carried out to explain overweight-related behaviors (i.e., Chapters 2-4), and 2) the intervention development and evaluation (i.e., Chapters 5-6). Finally, all the results, promises and pitfalls of applying the Intervention Mapping (IM) protocol in game-based intervention studies, practical 
constraints of mHealth research, implications for research and practice, and directions for future research are discussed in the General Discussion.

\section{PART I: The Needs Assessment}

Two main ORBs are dietary intake (DI) and physical activity (PA). For decades, differences in DI and PA have been explained by cognitive models such as the Theory of Planned Behavior (TPB), in which behavior is mainly explained in terms of behavioral intentions. However, only $12-20 \%$ of behavior can actually be explained by intentions. One possible explanation for this “intention-behavior gap" may be found in socioecological models. These models suggest that health behavior is the result of an interplay between motivational and contextual factors. Although previous explanatory studies on DI and PA (i.e., including both motivational and contextual factors) provide valuable insights to the field, their findings mainly rely on self-reported data and are, therefore, vulnerable to methodological issues (e.g., recall bias). A promising method that can be used to overcome these issues is mobilebased Ecological Momentary Assessment; mEMA.

Mobile-based Ecological Momentary Assessment. The promise of mobile-based Ecological Momentary Assessment (mEMA) is that it focuses on the collection of concurrent rather than retrospective self-reports, eliminating the memory-related biases that are inherent to retrospective reports. mEMA also realizes ecological validity by collecting data in real world settings. As such, it is likely that mEMA is a suitable method with which to monitor complex behaviors that fluctuate over time and are vulnerable to environmental influences, such as DI and PA.

Main Findings mEMA Studies (Chapters 2-4). The main findings of the feasibility and usability study presented in Chapter 2 showed that the target population (i.e., secondary vocational education students) generally carried their smartphone around on a daily basis, that the "mEMA" app was easy to use, and that the questions were well-displayed on their smartphones. These findings indicate that $\mathrm{mEMA}$ is a feasible and easy to use means of studying complex behaviors such as DI and PA. Subsequently, in Chapters 3 and 4, we used mEMA to explore how behaviors unfold over time. We showed that snack consumption (SC) was most likely to occur at the end of the day, whereas PA was most likely to take place during the late afternoon. Multivariate models indicated that SC was most likely to occur when students experience food craving. These models also showed that students were more likely to walk when they felt lively, to cycle when they felt happy or lonely, or when they were 
in the school environment, and to exercise when they felt satisfied or were in the presence of others. Although intention (i.e., the most proximal determinant of health behavior) was not associated with SC and PA multivariately, time lagged models, however, showed that intentions was positively associated to subsequent SC and PA.

Moreover, we compared registered compliance with self-rated compliance in all three mEMA studies. An overall comparison of registered mEMA compliance ( $43.8 \%)$ showed that students overestimated their compliance by approximately $26 \%$ as compared to average self-reported compliance data (70.6\%). Explanations for this poor use of mEMA may include a chaotic environment in which students were prompted (e.g., during lunch break), forgetfulness, or in turn, monitoring burden. This thin line between forgetfulness or monitoring burden results of prompting intensity. Too few prompts may result in forgetfulness and too many prompts may be perceived as annoying, both potentially decreasing participant compliance. As such, deliberate choices should be made in terms of signal-contingent recording, in which interference with daily activities should be taken into account and prompting intensity should be piloted.

\section{Part II: The "Balance It" Intervention (Chapters 5-6)}

The second part of this dissertation concerns the development and evaluation of "Balance It", a serious self-regulation game intervention targeting secondary vocational education students' DI and PA. Similar to existing online interventions (or e-Health interventions), serious games are designed to be educational and can be tailored to the individual. Serious games also hold the promise to be engaging, attention-captivating and intrinsically motivating. These games are assumed to increase compliance with the intervention and to decrease drop-out rates, as non-compliance and high drop-out rates have frequently been associated with limited effect sizes of online interventions. However, serious games are generally founded in game theory only. As it has been shown that a dual theoretical foundation in both health promotion and gaming can enhance intervention effectiveness, these theoretical fields were merged in the developmental process of "Balance It".

"Balance It". The "Balance It" intervention was mainly grounded in Self-Regulation Theory (SRT) with the aim of promoting a healthy lifestyle (i.e., through goal setting, self-monitoring, barrier identification and behavioral feedback), and the RE-AIM model and behavioral model by Fogg in order to stimulate intervention use simultaneously. In line with SRT and the RE-AIM model, performance 
objectives were formulated (i.e., IM Step 2). Subsequently, the most relevant and changeable determinants concerning DI and PA and intervention use were selected based on a review of the literature and previous mEMA studies (see Chapters 3 and 4), e.g., awareness, self-efficacy, skills, and triggers. As a product of these performance objectives and determinants, change objectives were defined, and both behavior change and persuasive methods were selected to facilitate the desired changes in DI and PA (IM Step 3). In IM Step 4, the intervention program "Balance It" was designed as a tailored, interactive multimedia game containing multiple game elements, such as "Tetris-shaped" building blocks that players can obtain by goal accomplishment and/or goal evaluation.

Main Findings "Balance It". The main findings of the "Balance It" pilot intervention showed short-term effects on secondary vocational education students' DI and PA. After four weeks, students in the intervention group reported a significantly stronger decrease in soft drink consumption as compared to the control group, and an increase in active transport (whereas the control group reported decreased use of active transport). No significant differences in change scores were found for fruit and vegetable consumption, snack consumption, and exercise between the two groups. However, only $28 \%$ of the intervention group reported that they had played "Balance It". Therefore, a second comparison was made between "intervention users" (i.e., $28 \%$ of the intervention group) and "non-users" (i.e., the remaining $72 \%$ of the intervention group $+100 \%$ of the control group).

Compared to "non-users", "intervention users" showed a significant increase in fruit and vegetable intake and decrease in snack consumption. A small increase in soft drink consumption was only marginally significant, and any increases in active transport and PA were not significant. User data (objective measures) showed that game players mainly chose to set goals in relation to decrease their snack consumption and to increase their moderate PA. Goal accomplishment was more likely when participants were motivated, and less likely when they experienced a lack of time. The games played mainly consisted of daily tasks and individual game play. Overall, "Balance It" was rated as moderately positive. 


\section{General Discussion}

Finally, all findings of the presented studies, promises and pitfalls of applying Intervention Mapping (IM) in mHealth interventions, implications for research and practice, directions for future research are provided in Chapter 7.

Promises and Pitfalls of Applying IM in mHealth Interventions. The IM protocol provided a strong foundation for the systematic development of "Balance It". With regard to serious game interventions, this theory and evidence-based foundation is grounded in both health promotion and gaming theory. Additionally, when applying IM to serious game design, a dual level implementation is required. These implementation levels are conceptualized as the contextual implementation level, indicating the context in which the intervention will be embedded (e.g., an existing system or structure), and the individual level, describing how the intervention should be implemented within the daily life of the intervention user to stimulate intervention use. These two dualities were encountered in the development of "Balance It", and resulted in a conceptual alteration of the IM framework.

Implications for Research and Practice. Based on the study findings and experiences from the developmental processes of "mEMA" and "Balance It", we recommended to use the IM protocol altered to mHealth research with a dual theoretical foundation and implementation level. We also advocate that trade-offs should be made when collaborating with professional designers (or other parties) to create "the best of both worlds".

Although several challenges still need to be overcome, "Balance It" shows potential to change health-related behavior among low SES youth. One such challenge is the limited compliance with "Balance It". Possibly, the prompting techniques that have been applied have been perceived as disruptive, limiting engagement with "Balance It". Therefore, the frequency of prompting should be piloted accurately to determine the most optimal prompting intensity. A second challenge relates to trade-offs between health promotion objectives and usability objectives, as compatibility issues between the two may arise during the developmental process behind serious games. One such example is the peer-support system of "Balance It" that has been placed outside the game (i.e., on an additionally created "Balance It" website) as a compromise between the need for peer support facilitation and game flow. 
Directions for Future Research. Future research may focus on triangulation of mEMA data, as mEMA allows for comprehensive data gathering, including self-reported measures, GPS data, and accelerometer data. As such, a more accurate understanding of complex health behavior in daily life may be achieved. Second, it is vital to gain a better understanding of (non-)compliance with mHealth applications. One possible explanation may be found in relation to the use of prompting, indicating a thin line between perceived monitoring burden (i.e., participants get annoyed due too many prompts) and forgetfulness (or "monitoring boredom", i.e., not enough prompts to keep participants engaged). As this breaking point may be different among different populations, future research on prompting intensity is wishful. Finally, further research on the use of serious games is needed to increase engagement with the intervention, for example by means of meta-interventions or noveallas.

\section{General Conclusion}

Although several limitations have been addressed, we 1) gained a better understanding of the dynamics in dietary intake and physical activity in secondary vocational education students' daily life, and 2) made a good attempt to change these complex behaviors among a population that is difficult to target. Overall, with the conceptually altered Intervention Mapping framework we have provided transparency on the systematic development of an mHealth intervention, called "Balance It". 

Nederlandse Samenvatting 
De toename van het aantal mensen met overgewicht of obesitas in de westerse maatschappij verandert niet alleen het straatbeeld, maar kan ook leiden tot ernstig fysieke en mentale problemen. Het hebben van overgewicht kan leiden tot gezondheidsgerelateerde problemen, zoals diabetes type II en cardiovasculaire aandoeningen, en deze brengen hoge gezondheidskosten met zich mee. Omdat overgewicht en bijbehorende gezondheidsproblemen het meest voorkomen in families met een lage sociaal economische status (SES) en minderheidsgroepen is het belangrijk om interventies te richten op deze lage SES groep. Met name jongeren uit deze groep zijn een belangrijke doelgroep voor gezondheidsinterventies, omdat overgewicht tijdens de jeugd een sterke voorspeller is voor overgewicht tijdens de volwassenheid. Aangezien veel jongeren met een lage SES een Middelbaar Beroeps Opleiding (MBO) volgen, hebben wij ons tijdens de volgende studies vooral gericht op MBO-leerlingen in Nederland.

Om het overgewichtsgerelateerde gedrag van MBO-leerlingen te begrijpen en te veranderen in hun dagelijkse context hebben wij het Intervention Mapping (IM) protocol toegepast. IM beschrijft de systematische ontwikkeling van een interventie in zes stappen. Het protocol begint met een needs assessment waarin een gezondheidsgerelateerd probleem (zoals overgewicht) geanalyseerd wordt (IM stap 1). Ook worden de gedrags- en omgevingsfactoren die dit probleem veroorzaken en de determinanten van deze veroorzakers geanalyseerd in deze eerste stap. Vervolgens worden de doelen voor veranderingen in gedrags- en omgevingsfactoren bepaald die moeten leiden tot een afname van het gezondheidsprobleem (IM stap 2) en worden er in IM stap 3 theoretische methodes geselecteerd om deze aan determinanten gekoppelde veranderdoelen te vertalen naar een interventieprogramma. Bij deze vertaalslag wordt extra aandacht besteed aan de randvoorwaardes waaraan de praktische vertaalslag van een methode moet voldoen om de effectiviteit van deze toegepaste methode te vergroten. Daarna wordt het interventie programma daadwerkelijk ontwikkeld en getest (IM stap 4), geïmplementeerd (IM stap 5) en geëvalueerd (IM stap 6).

In lijn met het IM protocol kan deze dissertatie ingedeeld worden in twee delen: 1) de needs assessment om de factoren die het eet- en beweeggedrag van MBO-leerlingen beïnvloeden in kaart te brengen (hoofdstuk 2-4), en 2) de ontwikkeling en evaluatie van de "serious self-regulation game" interventie genaamd "Balance It" (hoofdstuk 5-6). Tot slot worden alle resultaten, de beloftes en uitdagingen van IM toegepast op game-based interventies, praktische restricties van mHealth onderzoek, implicaties voor praktijk en wetenschap en richtingen voor toekomstig onderzoek uiteengezet in de algemene discussie (hoofdstuk 7). 


\section{DEEL I: De Needs Assessment}

Twee gedragingen die sterk gerelateerd zijn aan overgewicht zijn voedselinname en lichamelijke beweging. Decennia lang zijn verschillen in eet- en beweeggedrag verklaard op basis van cognitieve modellen, zoals de "Theory of Planned Behavior (TPB)", waarin gedragsintentie de voornaamste voorspeller is van daadwerkelijk gedrag. Echter is deze verklaarde variantie slechts $12-20 \%$, wat duidt op een "intention-behavior gap”. Een verklaring voor deze gap kan mogelijk gevonden worden in socioecologische modellen. Deze modellen suggereren dat gezondheidsgerelateerd gedrag het resultaat is van een wisselwerking tussen motivationale én contextuele factoren. Ondanks de waardevolle bevindeningen die zijn gedaan vanuit deze socioecologische benadering is een groot aantal van de studies in de huidige literatuur gebaseerd op zelfrapportage en daarom vatbaar voor methodologische problemen zoals recall bias. Een veelbelovende methode die deze methodologische problemen kan reduceren is mobile-based Ecological Momentary Assessment, oftewel "mEMA".

De grote belofte van mobile-based Ecological Momentary Assessment (mEMA) is dat data verzameld wordt in de situatie zelf in plaats van retrospectief waardoor de biases die gerelateerd zijn aan het menselijk geheugen worden geelimineerd. Ook de ecologische validiteit van de metingen wordt gerealiseerd door middel van mEMA, wat deze methode geschikt maakt voor het meten van complexe gedragingen in het dagelijks leven die onderworpen zijn aan zowel persoonlijke als contextuele factoren en bovendien flucteren over tijd. Daarom zijn hoofdstuk 2-4 gericht op de ontwikkeling van de app “mEMA” om eetgedrag (hoofdstuk 3) en beweeggedrag (hoofdstuk 4) te leren begrijpen.

De belangrijkste bevindingen van de feasibility en usability studie zijn gepresenteerd in hoofstuk 2 en tonen dat MBO-leerlingen over het algemeen hun smartphone dagelijks op zak dragen, dat mEMA makkelijk te gebruiken was, en dat de vragen die in mEMA gesteld worden goed weergegeven werden op hun smartphones. Deze bevindingen induceren dat mEMA bruikbaar is om complexe gedragingen zoals eet- en beweeggedrag te meten. Vervolgens wordt in hoofdstuk $\mathbf{3}$ en $\mathbf{4}$ beschreven hoe we mEMA gebruikt hebben om te onderzoeken hoe eet- en beweeggedrag veranderen over tijd. We hebben aangetoond dat snacks met name aan het einde van de dag worden geconsumeerd, en dat lichamelijke activiteit voornamelijk eind van de middag plaats vindt (bijvoorbeeld lopen, fietsen of sporten). Multivarite modellen hebben aangetoond dat snack consumptie het meest waarschijnlijk was wanneer studenten smachtten naar eten. Deze modellen toonden eveneens aan dat studenten meer geneigd waren om te gaan wandelen wanneer zij zich levendig voelden, dat zij 
meer geneigd waren om te gaan fietsen wanneer zij zich vrolijk of eenzaam voelden, of wanneer zij zich in de schoolomgeving bevonden, en dat studenten meer geneigd waren om te gaan sporten wanneer zij in het bijzijn waren van anderen. De multivariate modellen toonden geen relatie tussen intentie (de sterkste voorspeller van gezondheidsgerelateerd gedrag) en snack gedrag, en evenmin tussen intentie en lichamelijke beweging. Tijdreeksmodellen toonden echter wel een positieve relatie aan tussen intentie en zowel snack gedrag als lichamelijke beweging op het volgende tijdsmoment.

Bovendien hebben we een vergelijking gemaakt tussen geregistreerde compliance, de naleving van het $\mathrm{mEMA}$ protocol, en zelfgerapporteerde compliance in alle drie de mEMA studies. Een totale vergelijking van geregistreerde mEMA compliance (43.8\%) en zelfgerapporteerde compliance (70.6\%) toonde aan dat studenten hun compliance overschatten met circa $26.8 \%$. Slecht gebruik van mEMA zou verklaard kunnen worden door een chaotische omgeving waarin studenten zich bevonden wanneer zij een pushbericht ontvingen om mEMA in te vullen (bijvoorbeeld in een drukke schoolkantine tijdens de lunchpauze), doordat deelnemers vergaten om mEMA in te vullen, of doordat zij geïrriteerd waren geraakt door de pushberichten die verstuurd werden door de mEMA app. Wegens de dunne scheidingslijn tussen "forgetfulness" (het vergeten te reageren op een pushbericht) en "monitoring burden" (irritatie door het aantal ontvangen pushberichten) is het belangijk de intensiteit van het aantal pushberichten dat op een dag naar de app gebruiker wordt gestuurd goed af te stemmen op zijn/haar behoeftes en dagelijks ritme. Door te weinig pushberichten te sturen naar de app gebruiker kan hij/zij vergeten om terug te komen naar de app, maar door te veel pushberichten te sturen kan de app gebruiker geïrriteerd raken, wat in beide gevallen kan leiden tot een afname in de compliance. Daarom is het belangrijk om overwogen keuzes te maken omtrent de pushbericht-intensiteit, waarin interferentie met het dagelijks leven worden meegenomen en de pushbericht-intensiteit getest wordt bij de doelgroep.

\section{DEEL II: De “Balance It" Interventie}

Het tweede deel van dit proefschrift beschrijft de ontwikkeling en evaluatie van "Balance It", een serious zelf-regulatie game interventie gericht op het eet- en beweeg gedrag van MBO-leerlingen in Nederland. Serious games zijn net als bestaande online interventies (ook wel e-Health interventies genoemd) educatief en afgestemd op de behoeftes van het individu. Serious games hebben daarnaast de potentie om de spelers te boeien, hen te betrekken in het spel, hun aandacht vast te houden 
en de spelers intrinsiek te motiveren. Zodoende wordt verondersteld dat dit type games de compliance met de interventie kan verhogen en de uitval in interventiestudies kan verkleinen, en de totale effectgrootte van de interventie doen vergroten. Echter, ondanks de potentie van serious games ligt het draagvlak van deze games voornamelijk in de game theorie en niet in de gezondheidsbevordering en gedragsveranderingstheorie. Onderzoek heeft aangetoond dat een duale theoretische grondslag in zowel gezondheidsbevordering als gaming de interventie effectiviteit kan vergroten. Daarom is tijdens het ontwikkelingsproces van "Balance It" en multidisciplinaire aanpak gehanteerd.

In hoofdstuk $\mathbf{5}$ wordt de ontwikkeling van "Balance It" beschreven. De "Balance It" interventie is gebaseerd op de zelfregulatie theorie, het RE-AIM model en het gedragsmodel van Fogg. De grondslag in zelfregulatie theorie is toegepast om een gezonde leefstijl te stimuleren (via het stellen van doelen, het zelf-monitoren, het identificeren van barrières en het verschaffen van feedback op het gedrag). Het RE-AIM model en het "behavioral model" van Fogg zijn toegepast om tegelijkertijd het interventiegebruik te stimuleren. De performance objectives (IM stap 2) zijn opgesteld op basis van zowel de zelfregulatie theorie als de interventiegebruik-stimulerende modellen. Vervolgens zijn de meest relevante en veranderbare determinanten van eet- en beweeggedrag en interventiegebruik geselecteerd. Zo werden bewustwording, eigen effectiviteit, vaardigheden en triggers geselecteerd op basis van een literatuurreview en voorgaande mEMA studies (zie hoofstuk 3-4). Op basis van de performance objectives en bijbehorende determinanten zijn vervolgens change objectives gedefinieerd, en zijn zowel gedragsveranderings methodes als persuasieve methodes geselecteerd om de gewenste veranderingen in eet- en beweeggedrag te faciliteren (IM stap 3). In IM stap 4 is het interventie programma "Balance It" ontworpen. "Balance It" is een getailorde, interactieve multimediale game die verschillende game elementen bevat, zoals bouwblokken in verschillende vormen die spelers kunnen verkrijgen door hun doelen te behalen en/of hun doelen te evalueren.

De belangrijkste bevindingen uit de "Balance It" pilot studie staan beschreven in hoofdstuk 6 en tonen korte-termijneffecten aan op het eet- en beweeggedrag van MBO-leerlingen in Nederland. $\mathrm{Na}$ vier weken rapporteerden studenten in de interventiegroep een significant sterkere afname in frisdrank consumptie vergeleken met de controle groep, en een significante toename in het gebruik van actief transport (terwijl de controle groep afnam in actief transport). Er waren geen significante verschillen in verschilscores gevonden voor groente en fruit inname, snack consumptie en sport tussen de twee groupen. Echter rapporteerde maar 28\% van de interventiegroep daadwerkelijk 
"Balance It" gespeeld te hebben. Daarom is er een tweede vergelijking gemaakt tussen de interventiegebruikers ( $28 \%$ van studenten in de interventiegroep) en de nietgebruikers (de resterende $72 \%$ van de studenten in de interventiegroep $+100 \%$ van de controlegroep). Deze vergelijking toonde een significante toename in groente en fruit inname en een afname in snack consumptie aan bij de gebruikers van de interventie (ten opzichte van de niet-gebuikers en de controlegroep). De verschilscores in frisdrankconsumptie verschilden enkel marginaal significant, en de verschilscores in actief transport waren niet significant. Uit de gebruikersdata bleek dat spelers met name minder wilden snacken en meer wilden gaan wandelen en fietsen. De waarschijnlijkheid dat studenten hun doel behaalden werd vergroot wanneer zij ook daadwerkelijk gemotiveerd waren om hun doel te behalen, en gehinderd wanneer zij een gebrek aan tijd ervaarden. De studenten speelden met name spellen die een dagtaak betroffen en ze speelden het liefst alleen. Over het algemeen kan gesteld worden dat "Balance It" redelijk positief beoordeeld werd.

\section{Algemene Discussie}

Het IM protocol zorgde voor een sterk draagvlak voor de systematische ontwikkeling van "Balance It". De theoretische en empirische grondslag in de serious game ligt in zowel in de gezondheidsbevordering als in de game theorie. Bovendien is er sprake van een noodzakelijk duaal niveau waarop de implementatie plaatsvindt wanneer IM wordt toegepast in serious game design. Deze twee niveaus zijn geconceptualiseerd als het contextuele implementatie niveau, de context waar binnen de interventie geïmplementeerd wordt (zoals een bestaand schoolsysteem), en het individuele implementatie niveau, welke een beschrijving geeft van hoe de interventie geïmplementeerd zou moeten worden binnen het dagelijks leven van de interventie gebruiker om het interventie gebruik te stimuleren. Deze twee implementatie niveaus zijn opgenomen in de ontwikkeling van "Balance It" en hebben geleid tot een conceptuele aanpassing in het IM protocol.

Op basis van de bevindingen en opgedane ervaringen tijdens het ontwikkelingsproces van "mEMA" en "Balance It" bevelen we mHealth onderzoekers aan om de aangepaste versie van het IM protocol te gebruiken om zodoende meer nadruk te leggen op de duale theoretische en empirische fundering (in zowel gezondheidsbevordering als gaming literatuur) en het duale implementatie niveau (zowel op contextueel als individueel niveau). We pleiten er eveneens voor om trade-offs te maken gedurende het ontwikkelingsproces met professionele ontwikkelaars (of andere partijen) om zo het beste van twee werelden te creëren. 
"Balance It" heeft de potentie om gezondheidsgerelateerd gedrag te veranderen van jongeren met een lage SES. Echter zijn er eerst nog een aantal uitdagingen. Een van de "Balance It" uitdagingen is het beperkte gebruik van de app. De toegepaste prompt-technieken, dat wil zeggen het verzenden van pushberichten, zijn mogelijk storend geweest waardoor "Balance It" minder gebruikt werd. Daarom moet de prompt frequentie accuraat getest worden om de waargenomen prompt intensitiet te optimaliseren. Een tweede uitdaging is gerelateerd aan de afwegingen die zijn gemaakt tussen de bevordering van gezondheidsgerelateerd gedrag enerzijds, en het interventiegebruik anderzijds om de fusie van de twee type objectives te bevorderen. Zo is bijvoorbeeld het peer-support systeem van "Balance It" buiten de game geplaatst op een apart ontwikkelde "Balance It" website als oplossing voor de behoefte aan een peer support systeem (gezondheidsbevordering) en het bevorderen van de game flow (interventie gebruik).

Toekomstig onderzoek gericht op de triangulatie van mEMA data is wenselijk, omdat de combinatie van zelfgerapporteerde data, GPS data en versnellingsdata een veelomvattende dataset vormt. Zodoende kunnen we complex gezondheidsgerelateerd gedrag in de dagelijkse setting mogelijk beter verklaren. Ten tweede is het belangrijk om een beter begrip te krijgen van (niet) gebruik van mHealth applicaties. Een mogelijke verklaring voor niet-gebruik zou gerelateerd kunnen zijn aan de intensiteit van het aantal verzonden pushberichten en de dunne scheidingslijn tussen het vergeten om de app te gebruiken en irritatie aan de app. Omdat het "breekpunt" tussen deze twee uiteinden zou kunnen verschillen per doelgroep, is nader onderzoek naar het effect van prompting intensiteit wenselijk. Tot slot is toekomstig onderzoek naar het gebruik van serious games an sich wenselijk om manieren te vinden die de betrokkenheid met de interventie vergroten, bijvoorbeeld door middel van het includeren van meta-interventies of novella's.

\section{Algemene Conclusie}

We hebben met dit proefschrift bijgedragen aan 1) een betere verklaring van de dynamiek in het dagelijkse eet- en beweeggedrag van MBO-leerlingen, 2) het veranderen van deze complexe gedragingen, en 3) de transparantie van de systematische ontwikkeling van mHealth interventies en de aangepaste toepassing van het Intervention Mapping protocol tijdens de ontwikkeling van "Balance It". 



\section{Dankwoord}


Eindelijk... hij is af! En daarom wil ik graag van deze gelegenheid gebruik maken om een aantal mensen te bedanken die mij geholpen hebben tijdens mijn PhD.

Ten eerste mijn (co-)promotoren Gerjo, Theo en Pepijn. Wat ben ik jullie dankbaar voor de kans die jullie mij hebben gegeven. Het was een intensief project met veel uitdagingen en een zeer strakke planning in het laatste half jaar, maar het is gelukt! Na vier jaar was mijn proefschrift af. Mission accomplished Gerjo, bedankt voor het vertrouwen dat je me gaf, je treffende feedback op papers en het gevoel altijd welkom te zijn in Maastricht. Theo, jij liet mij vooral heel kritisch naar mijn eigen werk kijken en wist me op de juiste momenten te voorzien van een positieve noot, waarvoor ik je enorm dankbaar ben! En Pepijn, jou kan ik niet genoeg bedanken voor de tijd die je voor me vrij bleef maken, zelfs wanneer je agenda volledig vol liep met driedubbele afspraken. En we bleven lachen, ondanks verschillende uitdagingen die op ons pad kwamen hebben we mijns inziens een mooi product afgeleverd. Zorg je goed voor onze Bob?

Ik wil ook graag de leescommissie bedanken voor de tijd en aandacht die zij besteed hebben aan het beoordelen van mijn proefschrift, de MBO Raad en Stichting Test Je Leefstijl voor hun inzet en interesse tijdens de uitrol en implementatie van het onderzoek, Sylvia voor haar hulp in de uitrol van het onderzoek naar "Krachtvoer" waarin we een koppeling hebben gemaakt tussen "Balance It" en het reguliere "Krachtvoer" pakket, IJsfontein voor de prettige samenwerking en inzet gedurende de ontwikkeling van "Balance It", en natuurlijk alle contactpersonen, docenten en studenten op MBO-scholen in Nederland, want zonder jullie was het me nooit gelukt!

Lenneke en Sanne, wat ben ik blij met jullie als als collega's, als roomies, maar vooral als vriendinnen. Met jullie deelde ik de mooie momenten van mijn tijd als aio, maar ook in de minder mooie tijden stonden jullie voor mij klaar. Daarom vind ik het super fijn dat jullie op 18 maart mijn paranimfen zijn!

TNO'ers: bedankt voor de prettige tijd die ik bij jullie heb gehad in het Gorter en aan de Schipholweg. Ik heb genoten van mijn tijd als aio, als lid van de feestcommissie, de uitjes met het behavior change team, de koffiepauzes met o.a. Eline, Mariëtte, Lenneke, Britt, Gaby en Noortje, en de “wijze woorden" van Luuk wanneer het aan het einde van de dag alweer donker begon te worden: “je bent maar aio, ga naar huis" (waarvoor ik Luuk nog steeds dankbaar ben). Rosie en Gillian, jullie wil ik in het bijzonder bedanken voor jullie inzet tijdens het ontwikkelingsproces van "Balance It". Ik heb genoten van onze 
samenwerking! En Jet, jij verdient oprecht een extra bedankje als steun en toeverlaat sinds mijn sollicitatie bij TNO, dus bij deze!

UM'ers: Ik was niet heel vaak in het zuiden, maar als ik er was, was het altijd fijn om er weer te zijn! Jullie ontvingen me altijd met veel interesse en hielpen me opweg in het Limburgse. Mariëlla, ook jij verdient een extra bedankje. Op afstand of niet, op iedere vraag of incompleet formulier nam jij in no time contact met me op en losten we het samen op. Je bent een held!

CREATE EC members: You have become true friends. I absolutely enjoyed our shared interest in CREATE, and our collaboration and celebrations all over the world. You guys stimulated my international development and made me a bit "less Dutch". I also want to thank all CREATE facilitators and participants from the past few years for making the workshops as successful as they were. I absolutely enjoyed your commitment and hopefully we will meet again!

Vrienden en familie: Mam, Jillisa, Janieke, Thieme, Jo, Roos, Oom, Maartje, Alden my dear, Ber \& Patries, sjonnies, rode sokken en iedereen die ik nu vergeet.. Ookal waren jullie inhoudelijk minder betrokken bij het promotietraject, jullie waren minstens zo belangrijk! Jullie zorgden er voor dat ik een leven had naast mijn promotietraject. lets wat pas écht belangrijk is en wat ik nooit had willen missen! Ik ben dan ook super blij dat we er op 18 maart 2016 samen een mooi feestje van gaan maken. 



\section{Curriculum Vitae}




\section{Curriculum Vitae}

Jorinde Eline Spook was born in Haarlem, the Netherlands on the $1^{\text {st }}$ of July 1987. After completing secondary education at the Kaj Munk College in Hoofddorp in 2005, she started studying (work) Psychology at VU University Amsterdam. After finishing her Masters in 2009, she got accepted as a Social Research Master student (Communication Science) and finished her Research Masters degree in 2011. September 2011 Jorinde started as an external PhD Candidate of Maastricht University (Department of Work and Social Psychology) at

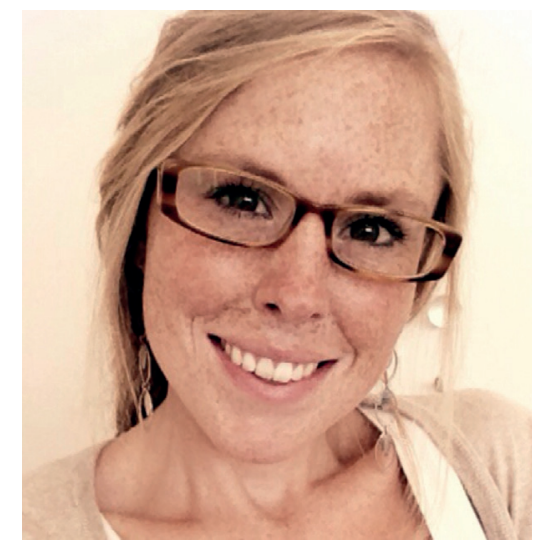
the Life Style expertise centre in Leiden. During her PhD, Jorinde has been awarded the prize for outstanding poster presented at the EHPS Conference three times (in 2012, 2013, and 2014). She finished her PhD project under supervision of Prof. dr. Gerjo Kok (UM), dr. Pepijn van Empelen (TNO), and dr. Theo Paulussen (TNO), and is now specialized in real-time measuring of complex healthrelated behaviors and in the systematic development of serious game interventions. Next to her PhD, Jorinde was an active EC member of CREATE (Collaborative REsearch And Training in the EHPS). She started as the CREATE Communications Officer in 2012 and became chair of the CREATE EC in 2015. After Jorinde finished her PhD in September 2015, she started as a Postdoctoral Researcher and Lecturer at Wageningen University and Research centre (chair group Strategic Communication) in October 2015.

Follow Jorinde on Linkedın: www.linkedin.com/in/jorindespook 



\section{Propositions accompanying the dissertation}

\section{KEEPING IT REAL: \\ Understanding and Changing Health Behavior in Daily Life}

Jorinde Eline Spook

March $18^{\text {th }}, 2016$

1. A dual theoretical foundation in both health and game theory is key in the systematic development of mHealth interventions (this thesis).

2. Prompting is an effective behavior change technique, but should be tailored to the target population's daily life (this thesis).

3. Mobile-based Ecological Momentary Assessment (mEMA) strategies have the potential to reveal the complexity of health-related behaviors (this thesis).

4. To create the best of both worlds, trade-offs have to be made (this thesis).

5. New games incorporating new ideas are vital for improving games for health and advancing our understanding of how games work and help players (T. Baranowski, 2014).

6. It is crucial to identify which environmental factors truly impact on physical activity and dietary behaviors and to carefully disentangle how these factors exert their influence on behaviors (E. de Vet, D. de Ridder, \& J. de Wit, 2011).

7. Het zal noodzakelijk zijn om meer gebruik te maken van inzichten buiten ons directe kennisveld, om zo een integratie van kennis tot stand te brengen en daarmee meer zicht te krijgen op de gedragsproblematiek (L. Lechner).

8. Normen zijn waardeloos op het gebied van obesitas preventie (S. Kremers).

9. Anticiperen op de gebruikerservaring is ook bij de planning van internetinterventies een essentieel onderdeel (R. Crutzen). 